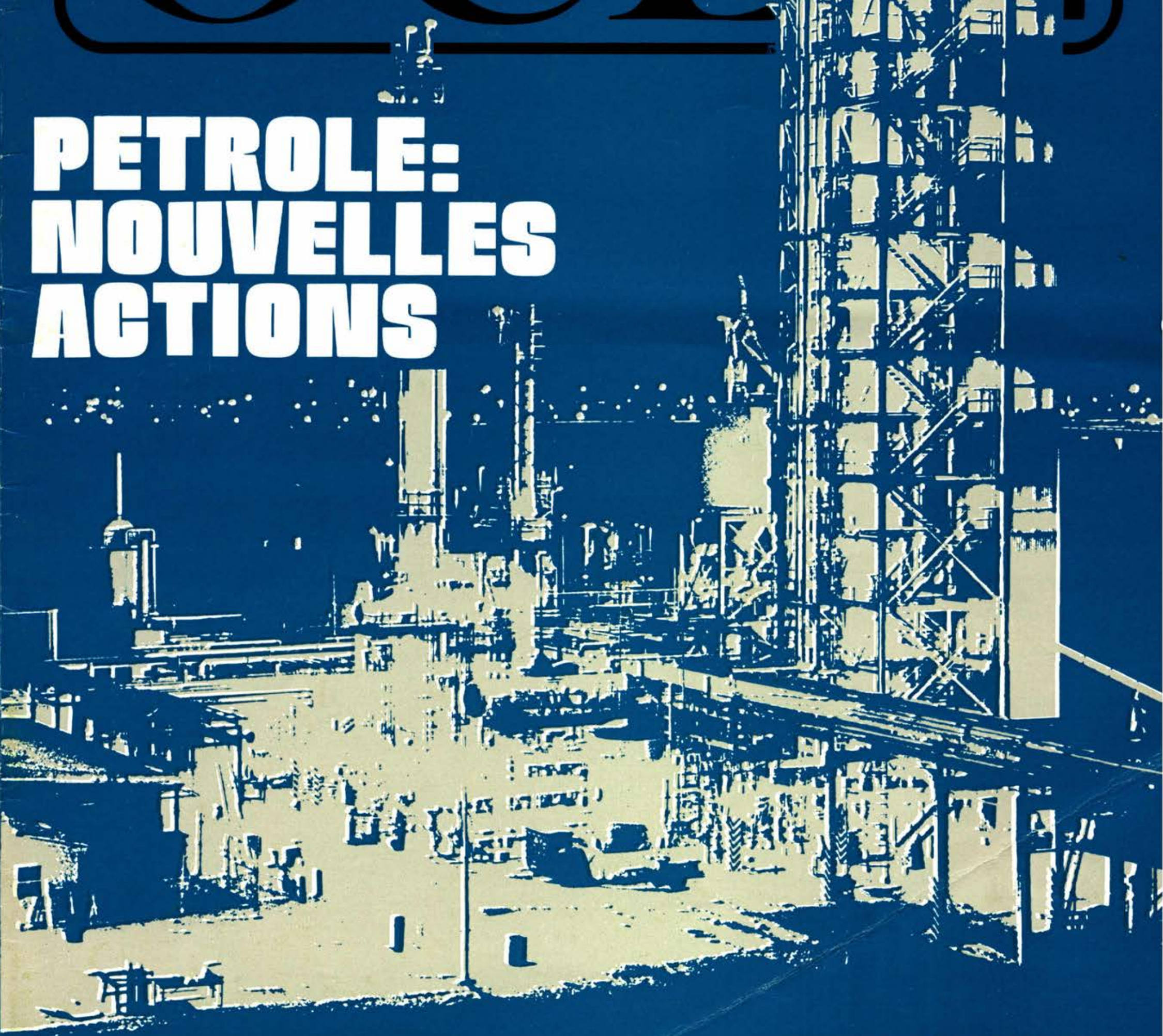




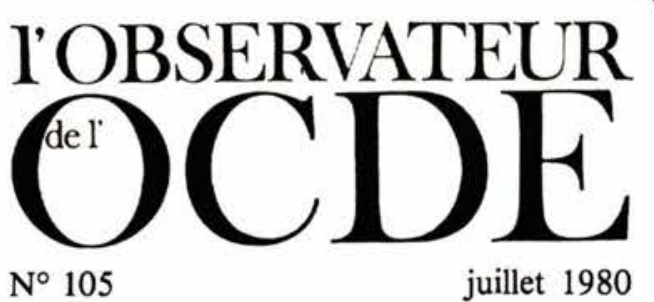

Publication bimestrielle en anglais et en français éditée par l'ORGANISATION DE COOPÉRATION ET DE DÉVELOPPEMENT ÉCONOMIQUES.

BUREAUX DE LA RÉDACTION: Service de l'information de l'OCDE, Château de la Muette, 2 rue André-Pascal, F 75775 PARIX CEDEX 16.

PERSPECTIVES ÉCONOMIQUES DE L'OCDE

Juillet 1980

NOUVELLES MESURES DANS LE DOMAINE

DE L'ÉNERGIE

UNE STRATÉGIE DE L'AIE POUR LA R-D ET D par Niels de Terra

Diagnostic et analyse d'un problème tenace

LE CHOOMAGE DES JEUNES

LES JEUNES ÉTRANGERS ET LE MONDE DU TRAVAIL

TENDANCES RÉCENTES DE L'AIE

L'AIDE EN 1979

commentée par John Lewis, président du

Comité d'aide au développement de l'OCDE

VERS UNE RÉGLEMENTATION INTERNATIONALE

DES PRODUITS CHIMIQUES TOXIQUES

RÉDUIRE LE BRUIT DES VÉHICULES A MOTEUR

LE NOUVEAU CENTRE DE BONN

LES NOUVELLES PUBLICATIONS DE L'OCDE

La photo de couverture ne représente pas une raffinerie de pétrole mais une usine de gazéification de charbon. L'utilisation de charbon pour la production de gaz ou de combustible liquide est des plus prometteuses pour l'avenir et la nouvelle stratégie de $R-D$ et $D$ de l'AIE accorde une haute priorité à ces techniques (p. 11). Sans de telles approches, la zone OCDE continuera à subir les effets du renchérissement du pétrole et des limitations de l'offre. La derniere série de hausses aura en 1980-81 des conséquences aussi bien récessionnistes qu'inflationnistes (p. 3). L'AlE fait des suggestions précises sur ce que chaque pays peut faire pour réduire sa dépendance à l'égard du pétrole ( $p .8)$ et établit des objectifs et des instruments de référence quant à la réduction des importations; elle analyse comment rompre le lien entre croissance économique et consommation d'énergie (p. 8).
Les articles sans copyright peuvent être reproduits à condition d'être accompagnés de la mention " Reproduit de l'OBSERVATEUR DE L'OCDE» en précisant la date du numéro. Deux exemplaires justificatifs devront être envoyés au rédacteur en chef. Les articles signés ne pourront être reproduits qu'avec la signature de leur auteur. L'Organisation n'est pas tenue de rendre les manuscrits qu'elle n'a pas sollicités.

Les articles signés expriment l'opinion de leurs auteurs et non pas nécessairement celle de l'OCDE.

Abonnement (un an):

F 36,00; £4.00; \$EU9.00.

Le numéro:

F 7,00; £0.80; \$EU 1.75 .

RÉDACTEUR EN CHEF: Jane Bussière

RÉDACTEUR EN CHEF ADJOINT:

Ulla Ranhall-Jeanneney

DiRECTION ARTISTIQUE ET TEChNIQUE: Marc Delemme

AssistanT:

Gérald Tingaud

RECHERCHE PHOTOGRAPHIQUE:

Silvia Lépot

Rina Maiden

Toute correspondance doit être adressée au rédacteur en chef.

PHOTOS: Couverture: Institute of Gas Technology, National Coal Association, ÉtatsUnis; page $13:$ B undesbildstelle, Bonn; page 14 : Sun Oil Company of Canada, Ltd.; page 15 : National Coal Board, Central Office of Information, Londres; pages 17 et 21 : Alain Nogues Sygma; page 22: Sven Oredson - Bildhuset; page 25 (haut): Jytte Bjorrepard; (bas): Paul Rimmerfors; page 28 : Bundesbildstelle, Bonn; page 32 (haut): Michel Philippot-Sygma; (bas à gauche): $A P$; (bas à droite): J. Fitzpatrick Service d'information australien; page 34: Georg Mynker, Bonn. 


\section{VERS UNE CROISSANCE PLUS ÉQUILIBRÉE Les points essentiels des PERSPECTIVES ÉCONOMIQUES DE L'OCDE Juillet 1980}

$\mathrm{U}$ The fois encore les perspectives à court terme de la zone de l'OCDE sont dominées par un choc pétrolier de grande ampleur. La hausse de plus de $130 \%$ (1) enregistrée par le prix du pétrole depuis la fin de 1978 a alourdi la facture pétrolière nette d'un montant équivalant à $2 \%$ environ du PNB; elle a fait monter le pourcentage de hausse des prix de plusieurs points, et aura d'ici à la fin de 1981 peutêtre réduit de quelque $5 \%$ le PNB de la zone de l'OCDE par rapport à ce qu'il aurait été autrement. La dernière vague inflationniste a déjà probablement culminé dans la plupart des pays de l'OCDE, et, à moins d'une nouvelle hausse des prix des produits pétroliers, les douze prochains mois devraient être marqués par une amélioration régulière. Dans le même temps, une récession s'est amorcée aux États-Unis et des signes de faiblesse de plus en plus nombreux sont apparus dans les économies de certains pays d'Europe.

Pour tous les pays de l'OCDE, il s'agit en priorité de poursuivre l'application de politiques budgétaires et monétaires restrictives afin de maîtriser l'incidence inflationniste du choc pétrolier. Si cette action est certes essentielle pour réaliser les conditions nécessaires au rétablissement d'une croissance équilibrée, elle ne constitue qu'un premier pas sur la longue route qui mène à une croissance plus satisfaisante et à un niveau d'emploi élevé. Le taux d'inflation sous-jacent est encore beaucoup trop élevé dans une bonne partie de la zone de l'OCDE et il représente une sérieuse contrainte pour la croissance. Les obstacles qui entravent le jeu des mécanismes du marché et le fonctionnement du commerce international continuent de provoquer des distorsions et des rigidités inopportunes et coûteuses par les pertes de productivité qu'elles entraînent et par la moindre adaptabilité au changement qui en résulte. Au demeurant, les économies des pays de l'OCDE sont exposées aux perturbations que pourraient provoquer de nouveaux chocs extérieurs trouvant leur origine sur les marchés pétroliers mondiaux. Cette situation requiert la mise en œuvre d'un ensemble de mesures visant à réduire l'inflation sous-jacente, à accroître le potentiel de production et à réduire la dépendance à l'égard de l'énergie.

\section{Évolution récente et prévisions}

Des résultats relativement satisfaisants avaient pu être obtenus en 1979 dans un certain nombre de domaines. Grâce à la mise en œuvre du programme d'action concertée de 1978 , le PNB s'était accru régulièrement à un rythme d'environ $3 \frac{1}{2} \%$ et la structure internationale des balances de paiements s'était nettement améliorée. L'inflation d'origine intérieure se situait aux environs de $7 \frac{1}{2} \%$ dans le groupe des grands pays, l'emploi augmentait et, dans plusieurs pays, le chômage baissait. Enfin, l'élément peut-être le plus encourageant de tous tenait à la vigueur de l'investissement des entreprises qui, dans plusieurs pays, augmentait à un rythme annuel plus rapide qu'au cours de chaque année ayant suivi la crise pétrolière de 1973-74.

Il est toutefois devenu manifeste en cours d'année que les progrès réalisés dans la voie d'une croissance mieux équilibrée et moins inflationniste étaient de plus en plus contrariés par la deuxième augmentation massive des prix pétroliers mondiaux, dont l'influence considérable pèse actuellement sur l'économie de la zone de l'OCDE. Le pre- mier effet en a été de faire monter les prix. Après une accélération de la croissance des prix à la consommation qui est passée, d'un rythme annuel de $81 / 4 \%$ au premier semestre de 1979 à environ $12 \%$ au premier semestre de cette année, le point culminant de la hausse pourrait actuellement avoir déjà été dépassé. Les répercussions de ces mouvements sur la demande réelle se font toutefois généralement sentir avec un certain retard. Le renchérissement du pétrole réduit le pouvoir d'achat des revenus intérieurs en augmentant la quantité de biens et de services qui doivent être offerts en échange de chaque baril de pétrole. Cette variation des termes de l'échange, qui est de nature à persister, sauf changement prononcé de la relation entre l'offre et la demande de pétrole de l'OPEP, a pour effet de réduire le volume de la dépense intérieure dans la zone de l'OCDE. Cette diminution a pour contrepartie une augmentation du revenu réel des pays de l'OPEP, mais il se trouve

(1) Prix à l'importation des pays de l'OCDE, juin 1980.

\section{INDICE DE PRIX IMPLICITE DE LA CONSOMMATION PRIVÉE DANS LA ZONE OCDE \\ Pourcentages de variation, taux annuels désaisonnalisés}

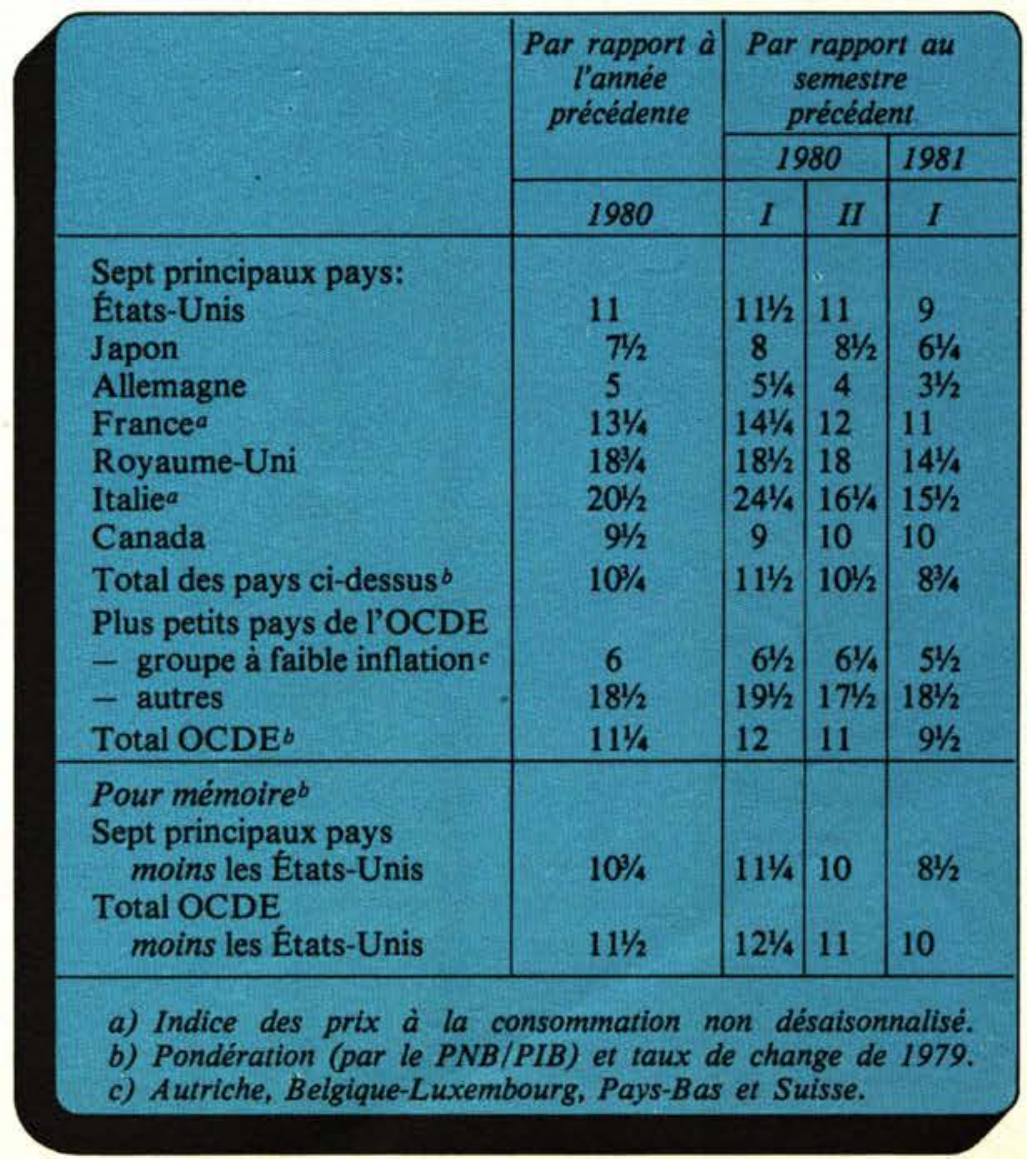


que, pour diverses raisons, ces derniers mettent en général un certain temps à dépenser ce revenu supplémentaire, de sorte que le total des dépenses intérieures et extérieures a tendance à baisser dans la zone de l'OCDE. Cet effet est compensé dans une certaine mesure par le flux d'épargne de l'OPEP qui, faisant retour dans le système bancaire de l'OCDE, tend à faire baisser les taux d'intérêt, à stimuler les emprunts privés et à soutenir la dépense. Toutefois, l'effet exercé sur la demande globale est sans doute, au moins pendant un certain temps, de caractère déflationniste. De plus, son influence déflationniste est renforcée par les politiques monétaires et budgétaires restrictives qu'il est nécessaire d'appliquer actuellement dans la quasi-totalité des pays de l'OCDE.

Compte tenu de ces diverses considérations et d'après les Perspectives économiques, on peut envisager une situation générale caractérisée par les éléments suivants :

- décélération modérée de la hausse des prix à la consommation, ramenée du taux de $12 \%$ observé dans les premiers mois de cette année à un peu moins de $10 \%$ au premier semestre de l'an prochain; les bons résultats auxquels paraissent avoir abouti jusqu'ici les efforts visant à absorber, sans accélération importante des revenus nominaux, les pertes de revenu réel provenant du renchérissement du pétrole, pourraient, semble-t-il, présenter un caractère durable (tableau 1)

- fléchissement du PNB de la zone de l'OCDE dans les derniers mois de cette année, suivi, au mieux, d'une croissance modérée l'an prochain; au milieu de 1981 , le niveau du PNB pourrait ne pas être plus élevé qu'actuellement (tableau 2)

- diminution de la productivité d'ici à la fin de l'année, suivie peutêtre d'une amélioration l'an prochain, mais s'accompagnant d'une tendance relativement faible de l'investissement non résidentiel du secteur privé pendant toute cette période

\section{CROISSANCE DU VOLUME DU PNB DANS LA ZONE OCDE} Pourcentages de variation, taux annuels désaisonnalisés

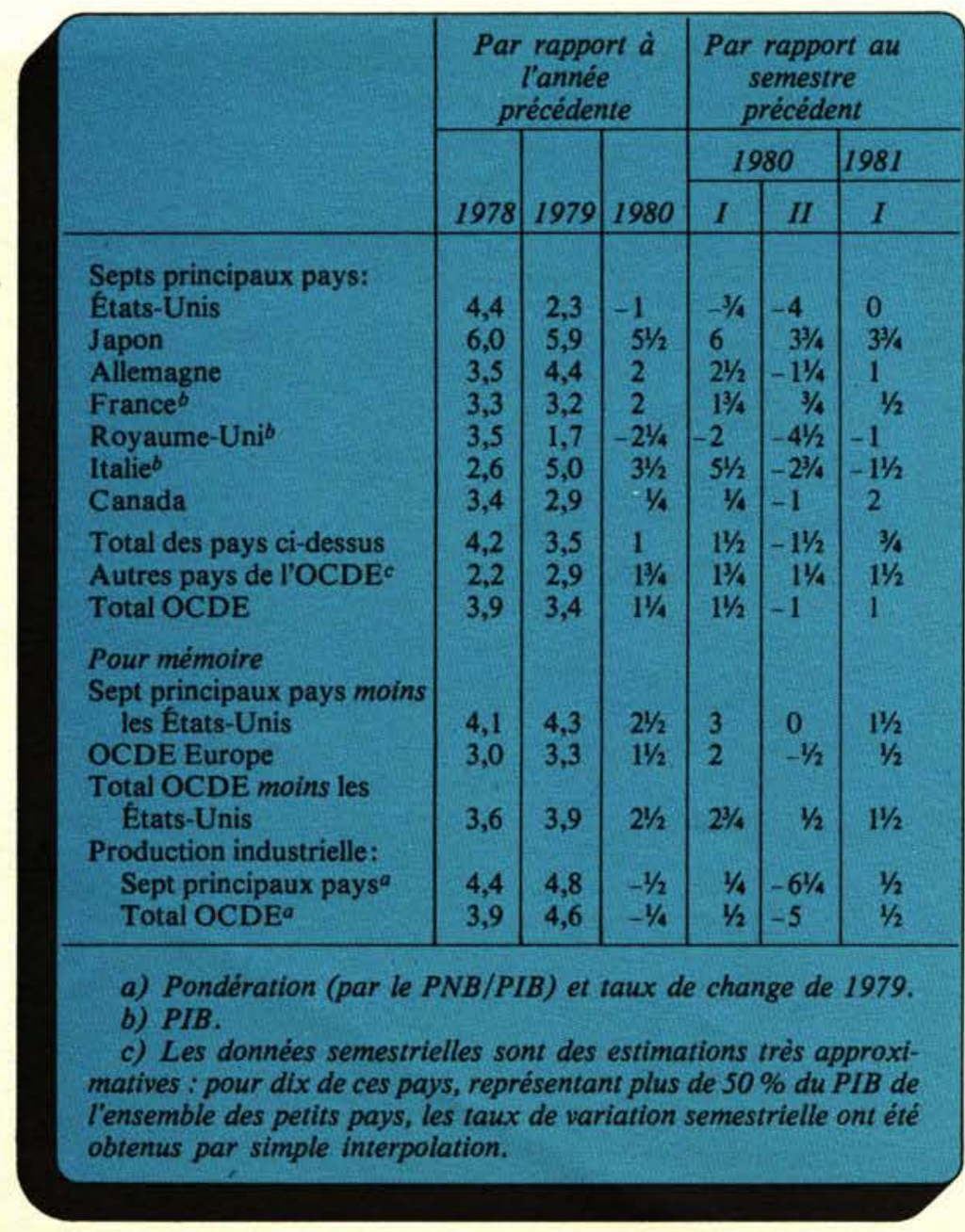

3. CROISSANCE DU VOLUME DU PIB DES AUTRES PAYS DE L'OCDE

Pourcentages de variation ${ }^{a}$

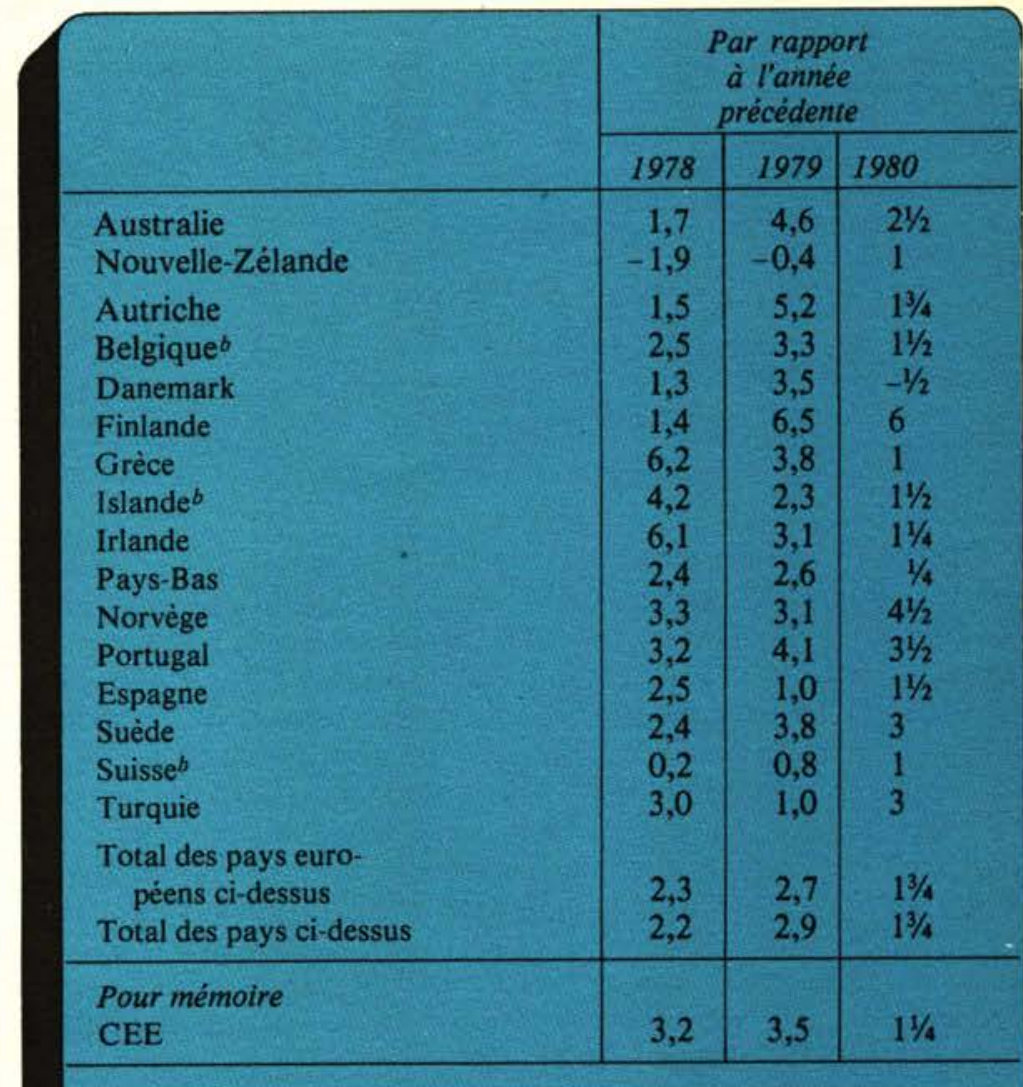

a) Pondération (par le PNB/PIB) et taux de change de 1979. b) PNB.

- augmentation du chômage malgré la faiblesse de la productivité, le nombre des chômeurs passant de 20 millions au premier semestre de 1980 à plus de 23 millions (près de $7 \%$ de la population active) au milieu de 1981 ; plus de la moitié de cette augmentation pourrait affecter les États-Unis

- persistance du déficit des opérations extérieures courantes de la zone de l'OCDE; ce dernier qui a probablement atteint un rythme annuel de près de 100 milliards de dollars au cours des premiers mois de cette année, pourrait cependant être ramené au-dessous de 50 milliards de dollars au premier semestre de l'an prochain (tableau 4) - grande diversité des résultats obtenus en matière de croissance et d'inflation; il est prévu qu'au cours des douze prochains mois le PNB baissera de $2 \%$ aux Etats-Unis, restera à peu près inchangé en Europe, mais augmentera de près de $4 \%$ au Japon; au milieu de l'an prochain, le taux d'inflation enregistré dans les grands pays pourrait être compris entre $31 / 2 \%$ (Allemagne) et près de $16 \%$ (Italie), l'écart étant encore plus large dans le cas des petits pays.

Si la zone de l'OCDE n'est pas soumise à de nouveaux chocs importants au cours des douze à dix-huit prochains mois, les économies des pays de l'OCDE seront en mesure d'absorber le choc pétrolier au prix d'un freinage de l'activité réelle moins fort qu'après l'augmentation des prix du pétrole de 1973-74, cette évolution s'accompagnant d'un ralentissement progressif de l'inflation dont le taux sous-jacent restera toutefois élevé(2). Jusqu'ici, le climat de confiance s'est dans l'ensemble maintenu dans le secteur privé. Toutefois, une perte de confiance, qu'elle résulte de perturbations survenant sur les marchés pétroliers internationaux ou de quelque autre cause, pourrait provoquer un fléchissement de la consommation pour un niveau de revenu réel donné si les consommateurs s'efforçaient de rétablir la valeur réelle de leur épargne ; d'autre part, une certaine incertitude accrue pourrait conduire les entreprises à réduire leurs projets

(2) Voir ci-après: ‘Les incidences de la situation pétrolière sur l'économie mondiale ». 
d'investissement, et peut-être déclencher un mouvement de déstockage.

\section{Objectifs de politique économique}

Pour la zone de l'OCDE, la perspective d'une augmentation du chômage, d'une inflation rapide, et d'un fléchissement du revenu réel par habitant n'est guère satisfaisante, quelles que soient les normes auxquelles on se réfère. Lors de leur réunion de juin 1980, les ministres des pays de l'OCDE ont souligné qu'étant donné la situation, le but essentiel devait être de rétablir la stabilité des prix et de promouvoir des conditions propices à une croissance durable à moyen terme de la production et de l'emploi. Ils sont convenus qu'à cet effet, il faut :

- "maitriser la poussée d'inflation imputable à la hausse des prix du pétrole et maintenir, ou obtenir, entre les coûts et les prix, un rapport suffisamment favorable pour qu'il soit intéressant d'investir

- cela fait, et à mesure que les efforts qui continueront d'être fournis pour réduire le taux d'inflation sous-jacent produiront leur effet, mettre en œuvre des politiques destinées à assurer un niveau d'activité suffisant pour que des investissements productifs soient à la fois nécessaires et rentables

- agir sur l'offre par des mesures positives visant à améliorer la productivité et la situation sur le plan de l'inflation, ainsi qu'à accroître le nombre des emplois, grâce à une augmentation de la part de l'épargne et des investissements productifs dans le PNB et à une amélioration du fonctionnement des marchés des produits, des capitaux et du travail $n(3)$.

\section{Politiques monétaires et budgétaires}

Dans l'avenir immédiat, il ne semble y avoir d'autre solution réaliste que de continuer de s'attacher en priorité à juguler la poussée d'inflation actuelle et à préserver la rentabilité des investissements productifs en combinant des politiques monétaires et budgétaires restrictives. Cependant, malgré des signes encourageants, on ne saurait guère aller au-delà d'un optimisme prudent quant à la mesure dans laquelle les politiques actuelles permettent d'absorber la hausse des prix du pétrole plus aisément et plus rapidement qu'après 1973. A cet égard, un dialogue avec les partenaires sociaux sur la nécessité d'accepter les conséquences de l'augmentation des prix du pétrole sur les revenus réels, revêt une importance considérable. Étant donné l'orientation nécessairement restrictive de la politique économique, la demande et la production pourraient finalement se révéler plus faibles que les prévisions ne le donnent à penser(4). Il pourrait être tentant de prendre rapidement des mesures en vue de soutenir la demande. L'expérience, acquise notamment depuis 1973, suggère toutefois que soutenir prématurément la demande peut rapidement conduire à une reprise de l'accélération de l'inflation et à une nouvelle période de restriction de la demande. De fait, les ministres «sont convenus que ce serait une erreur grave d'atténuer le caractère restrictif des politiques monétaires et budgétaires tant que la poussée actuelle d'inflation n'aura pas été maîtrisée de manière probante et que les conséquences de l'augmentation des prix du pétrole sur les revenus réels n'auront pas été intégralement absorbéesn.

Une fois absorbées les pertes imputables à la dégradation des termes de l'échange, les revenus réels et, par conséquent, la demande privée, auront tendance à faire preuve de plus de fermeté. En outre, le respect des taux d'expansion monétaire retenus comme objectifs devrait se traduire par une baisse sensible des taux d'intérêt lorsque la demande de crédits s'affaiblira et que les anticipations inflationnistes s'atténueront. Au demeurant, si l'on se réfère à la situation qui avait suivi la crise de 1973-74, la situation des profits est dans une large mesure préservée et les niveaux des stocks ne paraissent pas être excessifs. Bien qu'on puisse attendre de ces facteurs favorables du point de vue de la demande qu'ils enrayent la baisse et conduisent peut-être à une reprise modérée, il est douteux que, dans la plupart des pays, les politiques actuelles permettent d'escompter une reprise entièrement autonome. Les ministres ont donc été amenés à conclure que «lorsque le second choc pétrolier aura été effectivement absorbé, il sera peut-être souhaitable et possible de revenir à une politique budgé- taire moins restrictive qui soit compatible avec un sentier de croissance à moyen terme équilibrée. Les pays où le taux d'inflation sousjacent reste un problème majeur devront toutefois maintenir des politiques monétaires et budgétaires restrictives, tandis que les pays où le choc pétrolier a été absorbé et où le taux d'inflation sous-jacent est satisfaisant devraient modifier sans attendre l'orientation de leur politique économique si les éléments d'une reprise autonome se révèlent insuffisants ».

En ce qui concerne les paiements internationaux et les questions monétaires, les pays de l'OCDE devront inévitablement accepter de prendre en charge pendant un certain temps une part importante de la contrepartie des excédents des pays de l'OPEP. Il est encourageant à cet égard de constater que l'Allemagne et le Japon supportent actuellement, et pourront continuer de supporter, une large part du déficit

(3) Voir le supplément spécial de l'Observateur de l'OCDE, juin 1980. (4) Voir aRisques et incertitudes » dans la section Demande, production et emploi, Perspectives économiques de l'OCDE, juillet 1980.

\section{BALANCE DES OPÉRATIONS COURANTES DES PRINCIPAUX PAYS ET GROUPES DE PAYS DE L'OCDE}

Milliards de dollars, taux annuels désaisonnalisés

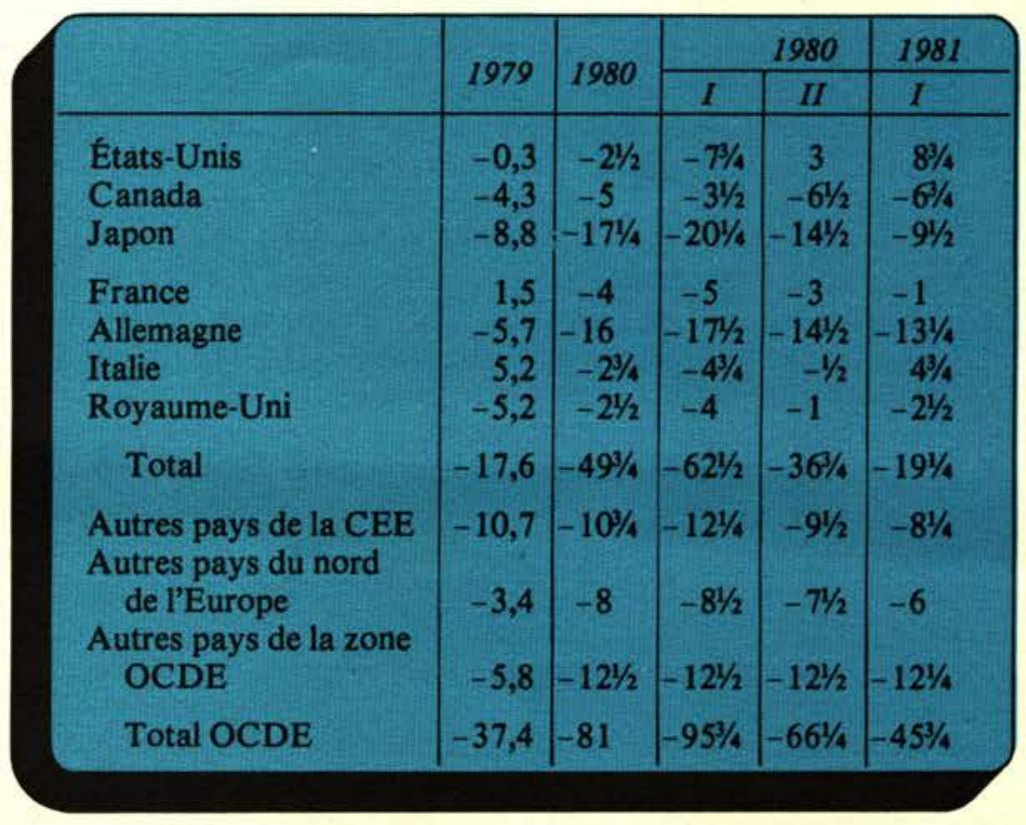

5. BALANCES DES OPÉRATIONS COURANTES DES AUTRES PAYS DE L'OCDE Millions de dollars

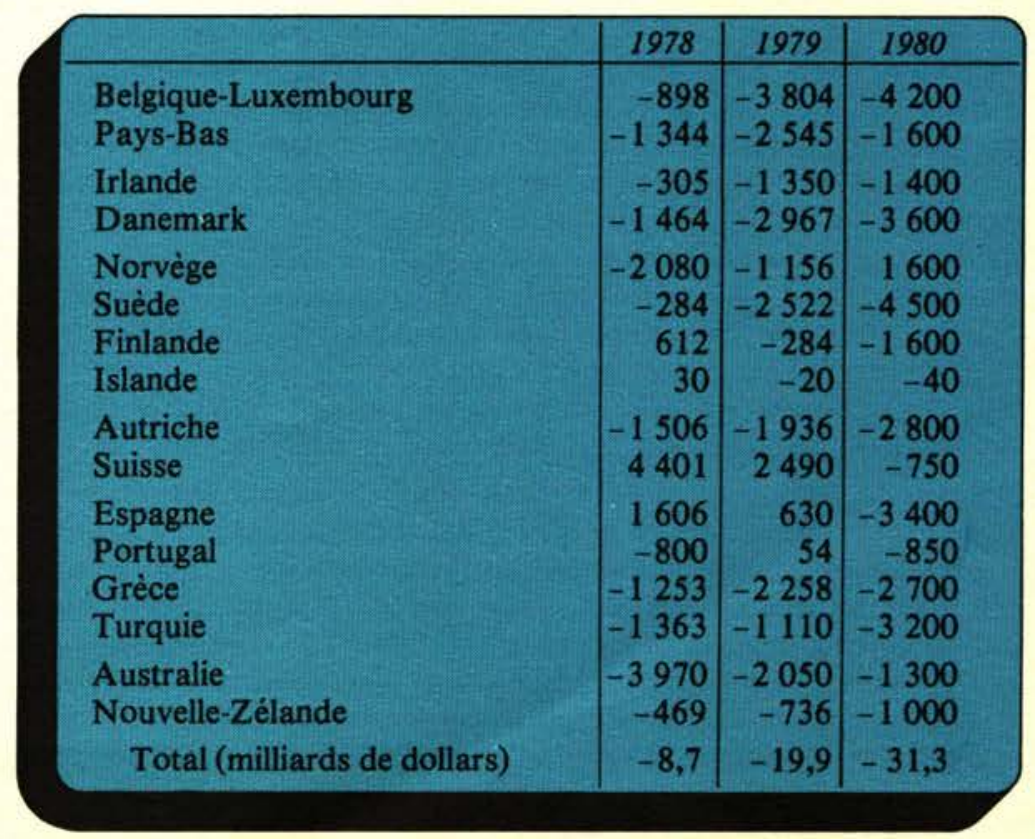


total de la zone. Il est moins satisfaisant qu'un groupe de quinze petits pays, représentant ensemble environ $15 \%$ du PNB de la zone, risque d'enregistrer encore un déficit global de près de 30 milliards de dollars (taux annuel) - c'est-à-dire plus de la moitié du déficit total de la zone de l'OCDE - au premier semestre de l'an prochain(5). Tous les pays devront mettre en œuvre des politiques destinées à réduire l'inflation et à favoriser l'adaptation structurelle à des prix accrus de l'énergie. A la réunion du Conseil de l'OCDE, les ministres ont reconnu que «les pays qui n'ont pas de problème de financement extérieur devraient s'abstenir de prendre des mesures visant spécifiquement à réduire les déficits des paiements courants $»$. On peut en déduire qu'il serait souhaitable que les pays où l'inflation est faible évitent autant que possible les dépréciations de leur monnaie et s'efforcent d'encourager les entrées de capitaux en utilisant d'autres moyens que la pratique de taux d'intérêt plus élevés que ne le requiert l'effort de stabilisation intérieure. De même, on peut en conclure que les pays où l'inflation est forte ne devraient pas aller trop loin dans les mesures visant à s'opposer à la dépréciation de leur monnaie, mais insister surtout sur les politiques de stabilisation.

A la suite de la dernière augmentation des prix du pétrole, les pays en développement non producteurs de pétrole doivent faire face à des besoins de financement extérieurs considérablement accrus. Cette situation appelle l'adoption de nouvelles mesures permettant de rendre plus sûrs les apports qui ne sont pas assortis de conditions libérales, en particulier à l'intention des pays en développement à revenu moyen qui éprouvent des difficultés croissantes de gestion de leur dette. Une aide accrue devra aussi être accordée aux pays à faible revenu qui n'ont pas la capacité d'ajustement voulue et qui ne peuvent accéder aux marchés des capitaux. Les pays de l'OCDE s'accordent à penser que les institutions financières internationales devraient jouer un rôle important pour fournir aux pays en développement des moyens financiers leur permettant de mettre en œuvre des mesures ordonnées d'adaptation et de changement structurels. L'octroi d'une aide, assortie de conditions libérales et d'un montant suffisant, aux pays les plus pauvres revêt une importance particulière pour prévenir un retard de leur développement. En plus de leurs efforts de financement du développement, les pays de l'OCDE devraient s'attacher à maintenir la liberté d'accès à leurs marchés de façon à faciliter l'accroissement des exportations des pays en développement.

\section{Action sur l'offre}

Il y a lieu de prendre des mesures visant à améliorer la productivité et la situation sur le plan de l'inflation afin d'aboutir, à moyen terme, à un taux de croissance plus élevé et à une augmentation des possibilités d'emploi. Cette action devrait être fondée sur des mesures agissant sur l'offre et visant à encourager l'expansion des capacités et à améliorer le fonctionnement des mécanismes du marché par l'élimination des distorsions et des rigidités, ainsi que sur des mesures propres à stimuler la concurrence. Il serait par exemple possible de faire disparaitre les distorsions structurelles qui freinent l'emploi et d'améliorer le fonctionnement des marchés du travail. Cette action pourrait contribuer à prévenir l'apparition de goulets d'étranglement dès que la croissance s'accélérera, et à améliorer les perspectives d'emploi à moyen terme, même s'il y a diminution de l'emploi à court terme. D'une façon plus générale, une stratégie faisant du relèvement de la productivité un élément essentiel de la croissance et de la lutte contre l'inflation, exige l'application, dans chaque pays, de politiques d'ajustement positives, et du maintien d'un système d'échanges ouvert. A cet effet, les gouvernements des pays de l'OCDE ont adopté, lors de leur récente réunion ministérielle, une nouvelle déclaration sur la politique commerciale.

Dans la mesure où il deviendrait possible, dans la poursuite des efforts menés pour réduire le taux d'inflation sous-jacent, d'assouplir avec le temps les orientations de la politique budgétaire, priorité pourrait utilement être donnée à des mesures réduisant les coûts et stimulant les investissements. Il importe en particulier d'intensifier l'investissement dans les domaines de la production d'énergie, de la substitution de combustibles et des économies d'énergie. En outre, ainsi qu'en sont convenus les ministres, la perspective de voir les niveaux de chômage rester élevés, et même augmenter, spécialement parmi les jeunes (voir page 16), «renforce les arguments en faveur du recours à des formules
COMPARAISON DE 1973/74 ET 1979/81

Sept grands pays

Taux annuels corrigés des variations saisonnières

Croissance du PNB (1) (échelle de gauche)

Inflation (indice des prix à la consommation) (échelle de gauche)

Variation des importations nettes de pétrole en pourcentage du PNB (échelle de droite)

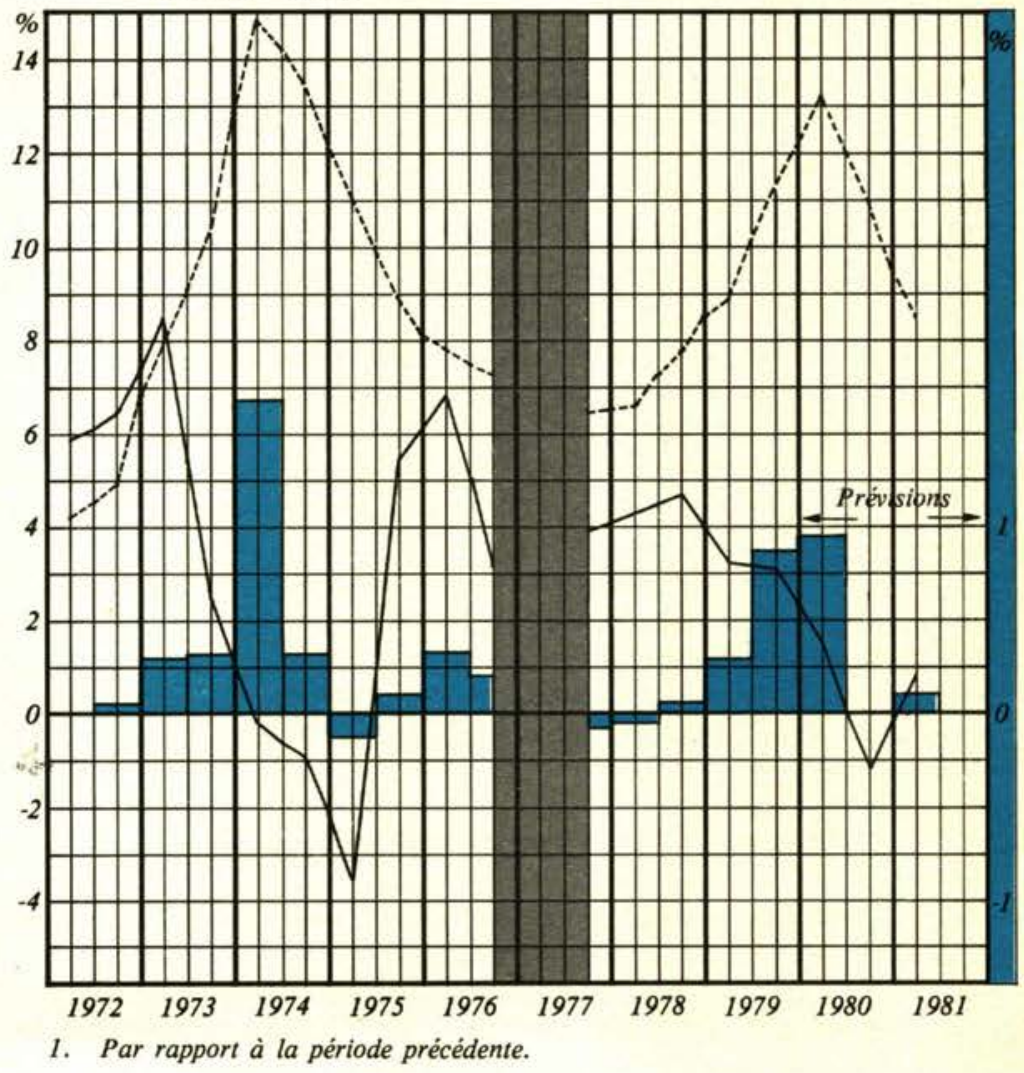

telles que des programmes de formation et de stages pratiques et, le cas échéant, de création d'emplois s'adressant à certains groupes cibles ou des mesures d'incitation visant à améliorer les possibilités d'emploi des groupes défavorisés ainsi que des travailleurs immigrés ». Des mesures de ce genre pourraient fournir un moyen de réduire le chômage qui exercerait relativement peu d'effets inflationnistes et aurait une bonne efficacité par rapport à leur coût. Il conviendrait toutefois dans de nombreux pays de veiller avec un soin particulier à ne pas adopter de mesures qui compromettraient la maîtrise à moyen terme des dépenses publiques.

\section{Énergie}

A la lumière des événements qui ont récemment affecté les marchés pétroliers et compte tenu des préjudices macro-économiques sérieux que pourraient causer de nouvelles et fortes hausses des prix du pétrole intervenant de manière soudaine, il importe de mettre en place les dispositions renforcées que le Conseil de direction de l'AIE réuni au niveau des ministres a proposées pour limiter les effets dommageables de perturbations à court terme du marché (voir page 8). En outre, ainsi que le Conseil de l'OCDE réuni au niveau des ministres en est convenu, une action vigoureuse s'impose d'urgence de la part des gouvernements en vue d'améliorer la situation de l'offre et de la demande d'énergie à moyen terme. Les pays de l'OCDE devraient utiliser de façon appropriée le mécanisme des prix, les mesures budgétaires ainsi que d'autres mesures de politique énergétique afin de renforcer et d'accélérer l'action voulue pour mener à bien les mutations structurelles nécessaires - par une utilisation plus rationnelle de l'énergie, par le remplacement du pétrole par d'autres combustibles et par un accroissement de la production d'autres formes d'énergie.

20 juin 1980.

(5) Voir les sections «Commerce extérieur et balances des paiements courants $»$ et "Évolution monétaire internationale », Perspectives économiques de l'OCDE, juillet 1980. 
Les Perspectives économiques de juillet 1980 comprennent une analyse détaillée de ce que l'Economist a appelé «la décennie de l'OPEP " et de l'influence que le pétrole peut avoir sur l'économie des pays de l'OCDE jusqu'à la fin de 1981. Plus spécifiquement, on $y$ analyse les changements des prix du pétrole et de l'énergie, la configuration de la production et de la consommation de pétrole et ses effets apparents sur les économies de l'OCDE (voir tableau), sur les pays de l'OPEP et les pays en développement non producteurs de pétrole. Quant aux perspectives à court terme, on peut s'attendre en 1980 à une réduction de la consommation de pétrole de la zone OCDE de quelque $2 \mathrm{mbj}$ et une baisse d'environ $3 \mathrm{mbj}$ des importations pétrolières nettes qui passeraient à $24,3 \mathrm{mbj}$.

Le résumé et les conclusions de cette section suivent.

\section{Le marché du pétrole}

- Si de faibles déséquilibres du marché pétrolier ont entraîné des accroissements très importants du prix du pétrole brut $(350 \%$ en $1973-1974,140 \%$ (1) en 1979-1980), la principale raison en est la faible élasticité-prix de la demande et de l'offre sur le court terme.

- L'économie de la zone OCDE est vulnérable à ces chocs pétroliers en raison de sa forte dépendance à l'égard du pétrole importé (un tiers des besoins énergétiques en 1979), dont le contrôle est passé des grandes compagnies pétrolières aux États producteurs.

- Néanmoins, les économies d'énergie induites par les augmentations de prix et soutenues par d'autres mesures réduisent la demande énergétique et ont conduit, avec le ralentissement de la croissance économique, à une situation moins instable du marché pétrolier au premier semestre 1980 .

- Le prix réel du pétrole et des autres formes d'énergie au stade de l'utilisation finale a beaucoup moins augmenté que le prix réel du pétrole à l'importation (bien que des augmentations doivent se produire en 1980), ce qui s'explique en grande partie par le fait que la part des taxes sur l'énergie dans les prix finals a fortement diminué.

\section{Aspects économiques}

- Les hausses du prix du pétrole de 1973 1974 ont été l'une des principales causes de la récession de 1974-1975, à laquelle une politique économique restrictive et d'autres facteurs ont cependant contribué.

- Les hausses du prix du pétrole de 1979 . 1980 ont jusqu'ici été surtout ressenties au niveau de l'inflation et des balances courantes, mais leurs effets déflationnistes, qui sont attendus mais qui ne se sont pas encore manifestés, pourraient n'être que retardés.

(1) Prix à l'exportation de l'OPEP, mai 1980.
- En 1973-1974, les agents économiques ont tenté d'éviter la diminution de leur revenu réel par un accroissement de leur revenu monétaire; cela a conduit à une spirale des salaires et des prix et à une compression des profits, et a provoqué un ralentissement marqué de l'investissement dans les années suivantes, qui a retardé la reprise.

- En 1979-1980, la réaction des salaires donne des signes de plus grande modération, de sorte que la perte de revenu réel sera peut-

\section{SIMULATION DES EFFETS SUR LA ZONE OCDE DE LA HAUSSE DU PRIX RÉEL DU PÉTROLE DEPUIS LA FIN DE 1978 a}

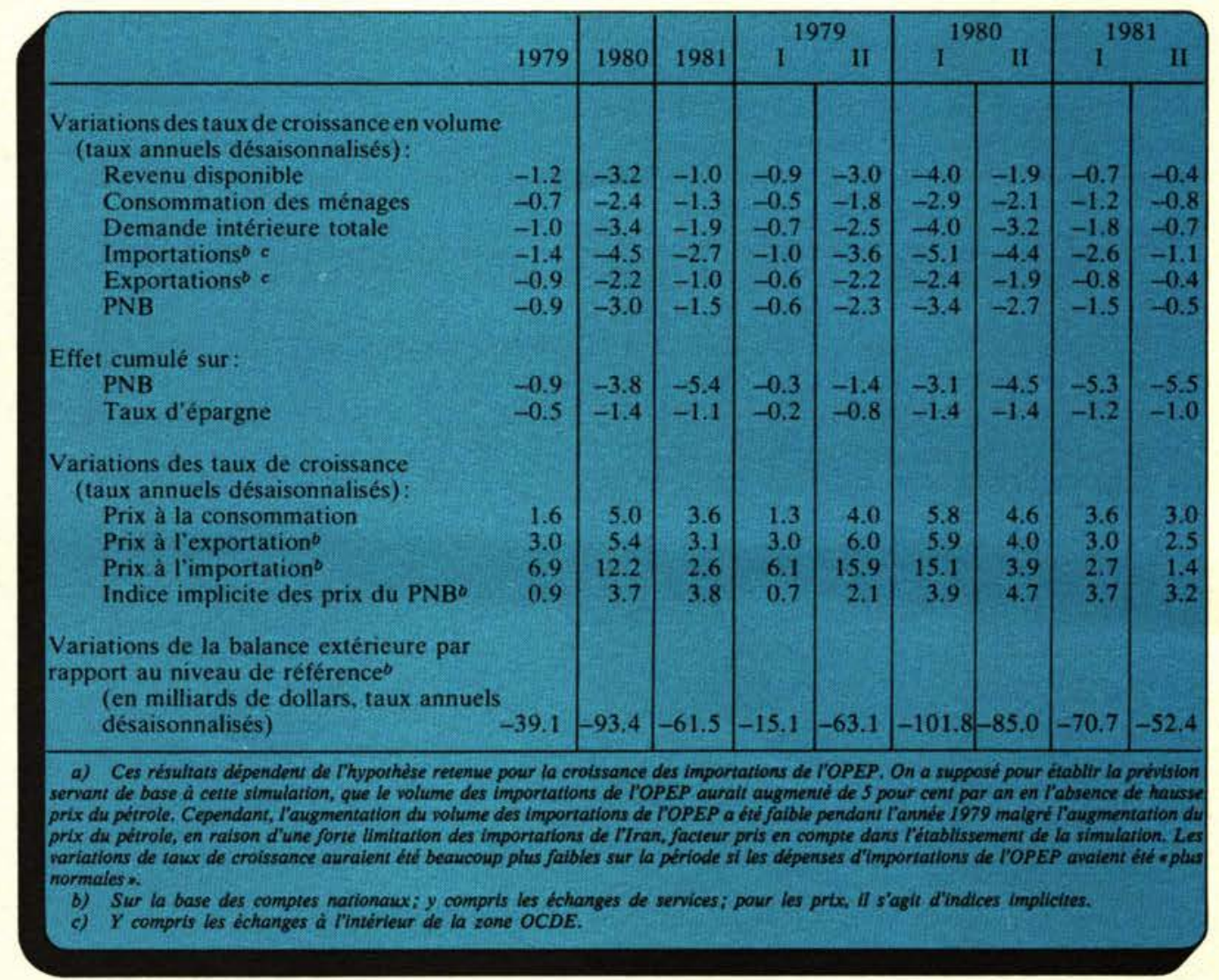

Selon les résultats de la simulation présentés dans le tableau ci-dessus, il apparait qu'en ce qui concerne la zone OCDE, une part importante de l'effet déflationniste des hausses des prix pétroliers de 1979-1980 ne s'est pas encore concrétisée, et que les répercussions de ces hausses pourraient être très vives tout au long de 1981. Pour 1981, le niveau simulé du PNB réel de la zone OCDE est inférieur d'environ $51 / 2 \%$ à ce qu'il serait dans d'autres conditions. Le niveau simulé du revenu réel disponible est aussi inférieur d'environ $5 \frac{1}{2}$ au niveau de référence de 1981 . Un fléchissement endogène du taux d'épargne des ménages atténue cependant les effets simulés sur la consommation des ménages durant 1980 et compense donc en partie l'effet déflationniste des hausses des prix de l'OPEP.

La croissance totale simulée du volume des exportations subit une variation positive, du fait des dépenses de remploi de l'OPEP, mais cet effet est plus que compensé par l'influence négative du ralentissement de la croissance sur les échanges commerciaux entre pays de l'OCDE. Les volumes des importations des pays de l'OCDE connaissent un fléchissement relatif, en raison principalement de la compression des revenus dans les pays. Étant donné qu'à court terme, l'élasticité-prix de la demande de pétrole est faible, les variations du prix à l'importation des produits énergétiques en provenance des pays de l'OPEP n'ont qu'un léger effet sur les volumes des importations pétrolières en 1981.

La balance extérieure réelle simulée de la zone OCDE s'améliore - compensant en partie les effets négatifs s'exerçant sur la croissance de la demande intérieure totale en 1980 - mais la dégradation des termes de l'échange reste la plus forte tout au long de la période. Par conséquent, la balance courante simulée de la zone OCDE accuse une détérioration d'environ 90 milliards de dollars en 1980. Cependant, les prix réels du pétrole restant par l'hypothèse inchangés en 1981, les dépenses de remploi que continuent de faire les pays de l'OPEP atténuent la dégradation supplémentaire de la balance courante pendant toute l'année 1981.

Fin 1981 , la hausse cumulée de $23 \%$ de l'indice de prix implicite des importations se traduit par une hausse cumulée des prix à la consommation de $10 \frac{1}{2} \%$ au cours de la même période. Les augmentations des prix de l'énergie d'origine intérieure, ainsi qu'une réaction salaires-prix, ne sont pas étrangères à cette hausse. Les prix des produits manufacturés exportés par les pays de l'OCDE réagissent à la hausse des coûts des facteurs d'origine intérieure ou importés, de sorte qu'à la fin de 1981 l'indice implicite des prix à l'exportation a atteint un niveau supérieur. de $12 \%$ au niveau de référence. 


\section{NOUVELLES MESURES DANS LE DOMAINE DE L'ENERGIE}

$\ll$ es très graves problèmes d'ajustement qui devaient se produire au milieu ou à la fin des années quatre-vingts se posent peut-être déjà de façon permanente et cela dans un climat lourd d'incertitudes politiques" - tel est l'avertissement qu'émet l'Agence internationale de l'énergie (AIE) dans sa dernière évaluation de la situation énergétique des pays membres (1). Telle est aussi la toile de fond des entretiens sur l'énergie qui ont eu lieu récemment, tout d'abord dans le cadre des réunions de I'AIE et de I'OCDE au niveau ministériel, ensuite au sommet de Venise.

La situation désordonnée des marchés pétroliers au cours de l'année 1979, pendant laquelle les prix du pétrole ont augmenté de $135 \%$ (de décembre 1978 à juin 1980), entraînera une diminution de la croissance économique (voir les Perspectives économiques, page 3 ) et, par conséquent, réduira - de la manière la moins souhaitable possible - la consommation de pétrole. Même dans ces conditions, les estimations actuelles de l'écart entre l'offre et la demande d'énergie sont supérieures à celles faites aussi récemment qu'en décembre dernier. Lors de leur réunion du mois de mai, les ministres de I'AIE sont convenus, sans fixer de chiffres, que les résultats à atteindre devraient «être largement en retrait " par rapport à l'objectif pour 1985, de 26,2 mbj (selon les chiffres les plus récents, les importations totales de 1979 ont été de 24,2 mbj). Le secrétariat de l'AIE estime, en se fondant sur les informations disponibles à l'heure actuelle, que les possibilités de réduction se situent aux environs de $4 \mathrm{mbj}$, compte tenu de toutes les incertitudes existantes. Les ministres sont également convenus de réduire le rapport entre le taux d'augmentation de la consommation d'énergie et le taux de croissance économique à 0,6 au cours de la décennie à venir (le chiffre correspondant était

(1) Politiques et programmes énergétiques des pays membres de l'AIE examen 1979.

\section{COMMENT RENFORCER LES POLITIQUES ÉNERGÉTIQUES ? LE SECRÉTARIAT DE LAIE ANALYSE LES POSSIBILITÉS DES DIFFÉRENTS PAYS}

Les prix intérieurs de pétrole devraient d'une manière générale refléter les prix internationaux. Les États-Unis devraient persévérer dans la voie de la suppression du contrôle des prix du pétrole et du gaz naturel. Le Canada devrait prendre des mesures aussi rapidement que possible pour porter les prix intérieurs du pétrole à un niveau qui favorise de nouvelles économies d'énergie, la substitution d'autres combustibles au pétrole et la mise en ceuvre de nouvelles sources d'énergie. Dans les pays qui possèdent d'abondantes réserves de gaz (Australie, Canada, Nouvelle-Zélande) et qui ont la possibilité d'accroître la production d'électricité à partir d'autres combustibles que le pétrole (Australie, Canada, Nouvelle-Zélande, Suede). la politique des prix de ces combustibles devrait tenir compte de l'opportunité d'encourager leur substitution au pétrole pour les utilisations appropriées.

Des efforts devraient être entrepris dans tous les pays (mais plus particulièrement aux États-Unis, en Italie, au Japon et aux Pays-Bas) pour réduire aussi rapidement que possible la production d'électricité à partir du pétrole. en le remplaçant par d'autres combustibles et en ne l'utilisant que pour les charges moyennes et les charges de pointe. Aucune centrale nouvelle ali- mentée au fuel ne devrait être autorisée, sauf dans des cas particuliers où il n'y aurait pas d'autre solution praticable. Les centrales existantes devraient fonctionner en utilisant au maximum des combustibles autres que le fuel.

\section{常}

Une action vigoureuse est nécessaire dans tous les pays pour réduire l'utilisation du pétrole dans l'industrie à d'autres titres que comme matière première nonénergétique. Une étude approfondie de la situation s'impose aux États-Unis, en Grèce, en Irlande, au Japon et aux PaysBas, où les prévisions indiquent que la consommation de pétrole pourrait s'accroitre rapidement, et en Allemagne, en Italie et au Royaume-Uni, où une action plus vigoureuse est peut-être nécessaire pour obtenir les résultats attendus.

$$
\text { 角 }
$$

Le pétrole devrait être remplacé par d'autres combustibles, employés directement (notamment pour le chauffage urbain) ou transformés en électricité, dans les utilisations résidentielles partout où l'infrastructure nécessaire existe ou peut être mise en place. Les pays qui ont actuellement recours au chauffage urbain ou qui envisagent d'y recourir devraient examiner la possibilité d'utiliser davantage le charbon à cette fin, en convertissant éventuellement des installations alimentées au pétrole. L'Allemagne, l'Australie, le Canada, I'Italie et le Japon devraient s'efforcer de remplacer le pétrole par le gaz naturel, les trois derniers pays cités en augmentant leurs importations. La Suede devrait encourager le remplacement du pétrole par l'électricité dans le secteur résidentiel. Le Royaume-Uni devrait prendre les dispositions voulues pour que ses plans actuels de remplacement du pétrole par le gaz naturel au cours des années quatre-vingts puissent être réalisés.

Tous les pays devraient mettre davantage l'accent sur des programmes vigoureux et complets d'économies d'énergie, pour encourager l'utilisation rationnelle et efficiente de l'énergie en général et du pétrole en particulier. Ces programmes doivent efficacement informer l'opinion des raisons pour lesquelles il faut économiser l'énergie et des moyens de le faire, et ils doivent aboutir a des résultats. En particulier, les normes d'efficacité de l'isolation thermique des logements devraient être examinées et relevées, si nécessaire. Les pays qui n'ont pas de programme de mise en conformité des systèmes d'isolation devraient envisager sérieusement d'en établir promptement, partout où les conditions climatiques le justifient. D'importantes économies d'énergie sont également possibles dans d'autres sec- 
supérieur à l'unité pour les dix ans qui se sont terminés en 1974), et de réduire la part du pétrole dans la demande totale d'énergie des $52 \%$ actuels - à quelque $40 \%$ d'ici à 1990 .

\section{Production pétrolière : inférieure aux prévisions}

L'expérience acquise au cours de l'année 1979 (voir encadré) a clairement montré que les quantités de pétrole fournies par I'OPEP seront très en retrait par rapport aux prévisions. "Le désir qu'ont exprimé de nombreux grands pays de l'OPEP de diminuer leur production est très réell, selon l'analyse la plus récente de I'AIE. L'Arabie Saoudite, le Koweit et Abu Dhabi limiteront vraisemblablement leur production; seul l'Irak semble susceptible de l'accroître. Par conséquent, la production de I'OPEP ne peut pas être prévue avec certitude, mais sera sans nul doute «notablement plus faible" que ne l'envisageaient les examens antérieurs (selon les prévisions pour 1985 de l'année dernière $37,5 \mathrm{mbj}$; selon celles de cette année $30,8 \mathrm{mbj}$ ).

Cette diminution des livraisons pourrait être compensée en partie par l'accroissement des fournitures du Mexique qui, en 1980 , doublera vraisemblablement sa production de 1978 et devrait pouvoir extraire de 3,5 à $5 \mathrm{mbj}$ en 1990; par contre, les autres pays non membres de I'OPEP ne fourniront sans doute pas beaucoup de pétrole au marché mondial. La production pétrolière de l'AIE, bien qu'en augmentation (surtout pour les gisements du versant nord de l'Alaska et de la Mer du Nord) n'a pas suffi à compenser le tarissement des régions productrices existantes.

Ces perspectives pessimistes en matière d'approvisionnements rendent plus urgentes les économies d'énergie et la mise au point d'autres sources énergétiques. Où en sont les différents pays à cet égard?

\section{Économies de pétrole : beaucoup reste à faire}

Certains résultats ont été obtenus. La croissance annuelle de la demande pétrolière, de $6 \%$ au cours des années soixante, a été ramenée à $0,7 \%$ seulement pendant la période $1973-1979$ et, en 1980, la demande devrait être inférieure de $8,6 \%$ à celle de 1979. (Le taux général d'augmentation de la consommation d'énergie a été de $0,8 \%$ de 1973 à 1979 alors que le taux de croissance du PNB était de 2,5\%.) C'est en 1978 que, pour la première fois, les importations pétrolières ont diminué alors que le PNB augmentait, mais cet écart reflète en grande partie l'accroissement de la production pétrolière de l'AIE. La consommation d'énergie par unité de PNB a déjà diminué, et l'utilisation des énergies de remplacement marque une progression d'environ $6 \%$ par an. "Une transition est en cours", souligne Ulf Lantzke, Directeur exécutif de l'AIE. "Par contre, le Secrétariat de I'AIE estime, et n'en fait pas mystère, que cette transition a été trop lente à s'amorcer». "Beaucoup a été fait depuis 1974, mais ce n'est pas teurs, notamment dans l'industrie et les transports, et des mesures appropriées devraient être prises pour faire en sorte qu'elles se matérialisent.

\section{光}

Dans le secteur des transports, des économies substantielles de pétrole peuvent être obtenues par l'amélioration continue des rendements énergétiques. Les pays qui mettent en ceuvre actuellement des programmes d'économies de combustibles (Allemagne, Australie, Canada, États-Unis, Japon, NouvelleZelande, Royaume-Uni, Suedel devraient les proroger pour faire en sorte que les rendements continuent à s'améliorer durant les années quatre-vingts, et examiner les dispositifs existants pour voir s'ils pourraient être renforcés. Ils devraient veiller à ce que ces programmes donnent des résultats, en les rendant obligatoires si nécessaire. Les pays qui n'ont pas établi de tels programmes devraient envisager d'en instituer

II faudrait examiner la possibilité de fixer des normes pour les véhicules a usage commercial et touristique, et tous les pays devraient revoir le niveau et la structure de leurs taxes sur les automobiles à faible rendement énergétique et sur l'essence. Les pays qui n'ont pas établi de limitations de vitesse plus strictes devraient envisager d'en imposer et veiller à leur application, afin de faire prendre conscience aux automobilistes de la nécessité d'économiser le carburant.

Des efforts plus vigoureux sont nécessaires pour accroitre la production charbonnière (Australie, Canada, ÉtatsUnis, pays qui devraient être prêts à augmenter encore leur capacité d'exporter des quantités substantielles de charbon). I'utilisation du charbon (Allemagne, Espagne, Italie, Japon, Royaume-Uni), et le commerce de ce produit. Dans ce dernier domaine, une attention accrue doit être portée à des arrangements contractuels à long terme, afin d'assurer la stabilité et la confiance requises pour exploiter de nouvelles mines et mettre en place les moyens de transport nécessaires. Une action positive doit être entreprise pour régler les problèmes d'environnement, action qui engloberait des projets de démonstration et d'autres mesures en faveur des technologies propres à réduire les incidences sur l'environnement.

Des efforts devraient être déployés pour accroitre les approvisionnements en gaz naturel en augmentant la production intérieure (États-Unis, Norvège, Royaume-Uni) et les importations (Allemagne, Autriche, Belgique, ÉtatsUnis, Italie, Japon, Suede). Dans tous les pays, les stratégies d'utilisation du gaz devraient permettre de réduire au minimum son emploi dans la production d'électricité et dans l'industrie, où il existe d'autres produits de remplacement du pétrole. Le Canada devrait poursuivre et renforcer ses efforts pour favoriser l'accroissement de la consommation intérieure de gaz naturel en remplacement du pétrole importé.

\section{से}

II faut intensifier les efforts pour exécuter les programmes nucléaires proje- tés et créer un environnement dans lequel il soit possible de discuter des questions nucléaires de façon objective et équilibrée, en tenant compte des considérations économiques énergétiques ainsi que des problèmes de sâreté et de prolifération (Allemagne, ÉtatsUnis, Italie, Japon), et pour rationaliser les réglementations relatives à l'autorisation de construire des usines nucléaires et aux autorisations permettant de mener des activités relatives au cycle du combustible nucléaire dans d'autres pays Membres.

Les activités de recherche et de mise en valeur des hydrocarbures devraient être renforcées afin de maximiser la production à long terme (Danemark, Espagne, États-Unis, Norvège, Nouvelle-Zélande, Pays-Bas, RoyaumeUni). Les possibilités d'accroitre la production par la récupération assistée devraient être activement recherchées.

\section{Des mesures devraient être prises} sans tarder afin d'accélérer le développement et la commercialisation de nouvelles technologies énergétiques, en particulier dans le domaine des économies d'énergie et des combustibles liquides et gazeux.

\section{光}

Les pays qui n'ont pas été cités nommément ci-dessus à propos de telle ou telle action spécifique devraient chercher attentivement, eux aussi, les possibilités de mettre en ceuvre des mesures nouvelles ou plus vigoureuses dans les secteurs qui peuvent les concerner. 
AIE : IMPORTATIONS NETTES DE PÉTROLE millions de barils par jour (soutes comprises)

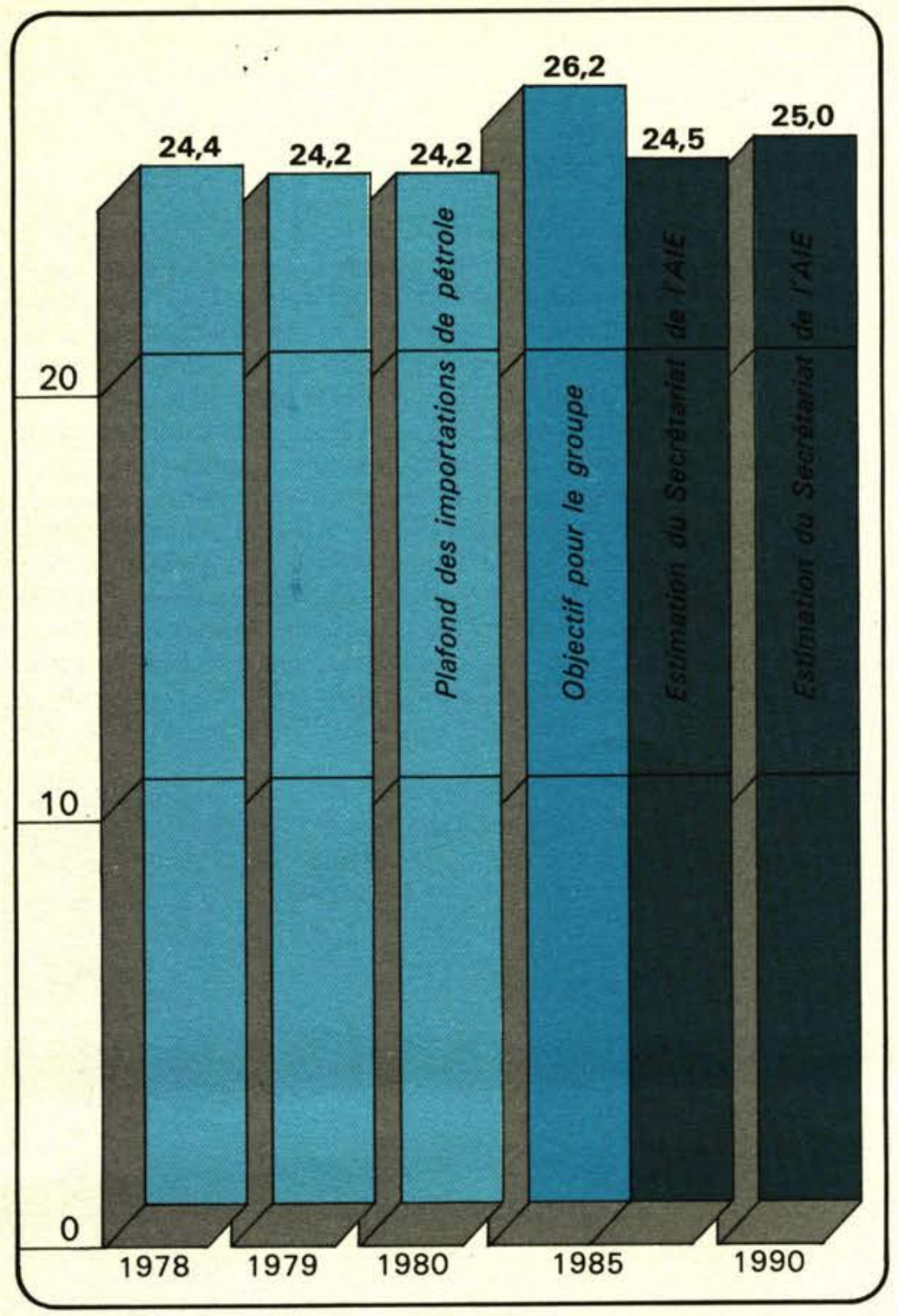

encore assez", ajoute le rapport officiel de l'AIE. Dans l'ensemble, la vigueur de la réaction ne correspond pas à la gravité de la situation ou à l'ampleur de la tâche. II n'y a pas de pays qui ne puisse et ne doive faire plus."

\section{Mesures à prendre}

Le Secrétariat a présenté à la réunion des ministres, fin mai, des suggestions très précises sur les mesures que chaque pays pourrait prendre afin d'améliorer sa politique (voir encadré). II a été convenu que "chaque ministre donnera le poids voulu à cette analyse lorsque son pays décidera des politiques énergétiques nationales requises». Les mesures seront également intensifiées à l'échelon international. Un comité consultatif vient ainsi d'être créé : il regroupe trente cadres supérieurs d'entreprises du secteur de la production et de l'utilisation du charbon ou des activités annexes; cet organisme doit rechercher la manière pratique de supprimer les obstacles considérables qui s'opposent à un accroissement notable de la production du charbon (qu'il est prévu de doubler d'ici à 1990, et de tripler d'ici à l'an 2000). Six groupes de travail vont présenter leurs rapports au mois de septembre et suggérer des mesures à prendre, par exemple pour agrandir les installations ferroviaires ou portuaires et résoudre les problèmes d'environnement.

C'est surtout dans le secteur nucléaire que les programmes nationaux d'approvisionnement en énergie ont pris du retard, et les ministres ont reconnu que «le développement de l'énergie nucléaire, dans des conditions appropriées tenant compte des progrès accomplis dans le cadre de l'évaluation internationale du cycle du combustible nucléaire (2), est indispensable pour assurer les mutations structurelles à moyen termen.

Un nouvel élan politique doit être donné à la recherche, au développement et à la démonstration (R-D et $D$ ) ainsi qu'à la commercialisation des technologies nouvelles qui présentent encore trop de risques pour attirer des investissements importants. L'AIE a mis au point une stratégie de R-D et D qui fixe les priorités en matière de recherche; les ministres ont décidé que cette stratégie servirait à fixer les priorités nationales et les niveaux de financement ainsi que l'ordre de priorité concernant les projets à réaliser en collaboration sur le plan international. Un comité de l'AIE examinera les efforts nationaux en matière de R-D.

Un système de consultation sur les politiques de stocks entre les gouvernements et entre les gouvernements et l'industrie pétrolière, a été adopté et sera utilisé en 1980 pour faire face à la situation du marché pétrolier; les détails restent toutefois encore à fixer et des lignes directrices doivent être élaborées par le Conseil de direction de I'AIE. L'on considère que la concurrence provoquée par la constitution des stocks a sans doute contribué de façon très importante au désordre des marchés en 1979; cette année, les niveaux des stocks sont restés jusqu'ici virtuellement inchangés et n'ont pas subi leur importante baisse hivernale habituelle, ce qui permet de penser qu'ils continueront à croître en 1980.

\section{Instruments de référence et plafonds}

Parmi les dispositions à court terme, l'AIE a examiné chaque trimestre les plafonds d'importation pétrolière fixés pour 1980. Au premier trimestre, l'examen a montré que tous les pays prévoyaient de respecter les limites fixées en décembre 1979 (3). Comme les besoins d'importation et la production sont, du moins à I'heure actuelle, à peu près en équilibre, il ne paraît pas nécessaire de réviser les plafonds existants ou d'en fixer pour 1981. Mais les prix continuent d'augmenter malgré cet équilibre presque parfait, ce qui montre bien à quel point le marché pétrolier est mouvant et avec quelle facilité des événements analogues à ceux d'Iran pourraient se produire. "A court terme, l'offre et la demande ne réagissent que relativement peu aux prix et, dans un marché instable, les premières pressions à la hausse sur les prix ont tendance à être renforcées par un comportement spéculatif », note le rapport de I'AIE.

Un système souple d'instruments de référence et de plafonds a par conséquent été mis au point, aussi bien pour mesurer les progrès accomplis dans la réalisation des mutations structurelles permettant d'atteindre les objectifs fixés pour 1985 et 1990 , que pour pouvoir faire face à bref délai à une éventuelle détérioration du marché pétrolier.

- Les besoins en pétrole de chaque pays seront examinés tous les ans.

- En période de fonctionnement normal du marché, ces estimations serviront d'instruments de référence pour mesurer les mutations structurelles par comparaison avec les objectifs à moyen terme. Elles seront également comparées aux perspectives à court terme de l'offre de pétrole de façon à permettre de suivre l'évolution du marché pétrolier.

- En cas d'apparition de signes d'une tension imminente du marché, les ministres se réuniront à bref délai pour prendre une décision sur l'utilisation des plafonds individuels d'importation de pétrole, fixés en partie sur la base des estimations de référence. "Les plafonds représenteront un engagement politique indiquant le degré d'auto-limitation que les différents pays sont prêts à

(2) L'évaluation internationale du cycle du combustible nucléaire a débuté en octobre 1977; le rapport correspondant a été publié en février 1980.

(3) Voir I'OBSERVATEUR DE L'OCDE n० 102, janvier 1980. 
s'imposer en cas de tension du marché afin d'empêcher que l'insuffisance des approvisionnements n'entraîne une concurrence acharnée», indique le communiqué ministériel. Un système d'ajustement est également prévu pour permettre de modifier les plafonds et les objectifs si l'évolution du marché pétrolier l'exigeait.

\title{
BILAN 1979
}

\section{Production}

Le déficit global d'approvisionnement pétrolier provoqué par l'arrêt des exportations iraniennes n'a jamais dépassé $4 \%$ et, pour les six premiers mois de l'année, a représenté $1 \%$. Au second semestre de 1979, la production de l'OPEP a été supérieure à la consommation et atteint le chiffre record de 31,6 mbj sur l'année.

\section{Consommation}

Après l'arrêt des exportations iraniennes, les gouvernements des pays membres de l'AIE ont décidé, en mars, de réduire leur demande de pétrole sur les marchés mondiaux de $2 \mathrm{mbj}$ (environ $5 \%$ de la consommation) par rapport aux quantités précédemment prévues. Les résultats ont varié d'un pays à l'autre, mais la consommation totale de l'AIE a été réduite de 0,9\% par rapport à celle de 1978 et de 2,6\% par rapport à celle prévue antérieurement pour 1979. Au cours du quatrième trimestre, les économies ont représenté en moyenne $5,7 \%$ de la consommation précédemment prévue. Six pays (Autriche, États-Unis, Luxembourg, Nouvelle-Zélande, Suisse et Turquie) ont réalisé des économies voisines de $5 \%$ ou même dépassant ce chiffre. Le Danemark a économisé plus de $3 \%$. Dans six pays (Allemagne, Belgique, Canada, Grèce, Japon et Royaume-Uni), la consommation pétrolière a été légèrement inférieure aux niveaux prévus. La consommation des sept autres pays a dépassé les niveaux prévus. Comme certains pays de l'AIE ont augmenté leur production pétrolière en 1979 , la réduction de la demande pétrolière sur les marchés mondiaux a été supérieure à la diminution de la consommation.

\section{Mutations structurelles}

Les quantités de pétrole brut auxquelles les grandes compagnies pétrolières internationales ont directement accès et qui font l'objet d'échanges internationaux ont diminué, passant de $16,9 \mathrm{mbj}$ à 14,5 . Ce déficit a été comblé par des négociants en pétrole brut qui achètent directement aux producteurs et vendent à l'industrie pétrolière, et par les compagnies pétrolières nationales augmentant leurs achats directs de brut; ces transactions ont souvent été effectuées aux prix du marché libre ou à des prix voisins de ceux pratiqués sur ce marché, avec l'acquiescement direct ou indirect des gouvernements.

\section{Prévisions pour 1980}

- La consommation pétrolière de l'AIE devrait diminuer d'environ $1 \mathrm{mbj}$.

- Les quantités fournies par des pays non membres de l'OPEP devraient augmenter de $0,8 \mathrm{mbj}$.

- En l'absence de toute nouvelle constitution de stocks, la demande de pétrole de l'OPEP s'en trouverait diminuée de près de $3 \mathrm{mbj}(1,2 \mathrm{mbj}$ en 1979).

- Toutefois, les quantités offertes pourraient, elles aussi, diminuer notablement sous l'influence des difficultés politiques et des problèmes de fixation de prix en Iran, et de baisses de production possibles dans d'autres pays.

- Dans l'ensemble, un petit déficit d'approvisionnement de $0,7 \mathrm{mbj}$ paraît possible. En tout cas, la situation devrait rester instable et sujette à de nouvelles perturbations.

\section{UNE STRATEGIE DE L'AIE POUR LA R-D ET D}

\author{
par Niels de Terra (1)
}

\begin{abstract}
Une nouvelle stratégie en matière de $R-D$ et $D$ qui établit les priorités de recherche a été mise au point par I'AIE (2). Les ministres des pays membres de l'Agence sont convenus de s'en servir comme guide pour les priorités et le financement au niveau national. L'article qui suit décrit les principaux éléments de cette stratégie.
\end{abstract}

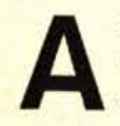

I'heure actuelle, il semble bien que la transition graduelle et sans heurts que l'on envisageait encore il y a seulement deux ou trois ans ne sera pas réalisable. La nouvelle urgence donne une plus grande proéminence au rôle central que des économies d'énergie nouvelles et des technologies du côté de l'offre peuvent jouer dans la réduction de l'utilisation de pétrole.

Les gouvernements des pays membres de l'AIE dépenseront, en 1980, plus de 7 milliards de dollars pour les travaux de recherche, développement et démonstration (R-D et $D$ ) en matière d'énergie. On peut dès lors se demander si ce niveau de dépenses et les priorités actuelles permettront de disposer commercialement, dans la mesure et au moment voulus, des technologies les plus utiles.

Cette question a été posée début 1976 par le Conseil de direction de I'AIE qui a ensuite lancé un vaste projet international visant à mettre au point une stratégie de $R-D$ et $D$ en matière d'énergie qui serait fonction des besoins de l'ensemble des pays membres de l'Agence.

\section{L'analyse}

Pour disposer de la base analytique nécessaire à la mise au point de cette stratégie, on a entrepris une analyse des systèmes qui a duré trois ans et représenté un travail de près de 100 hommes-années. L'analyse a été effectuée par des équipes

(1) Bureau de recherche, développement et applications technologiques de l'AIE.

(2) Agence internationale de l'énergie. 
PROJET DE STRATÉGIE R-D ET D DE L'AIE Importations nettes de pétrole de sources traditionnelles deux scénarios (millions de tonnes)

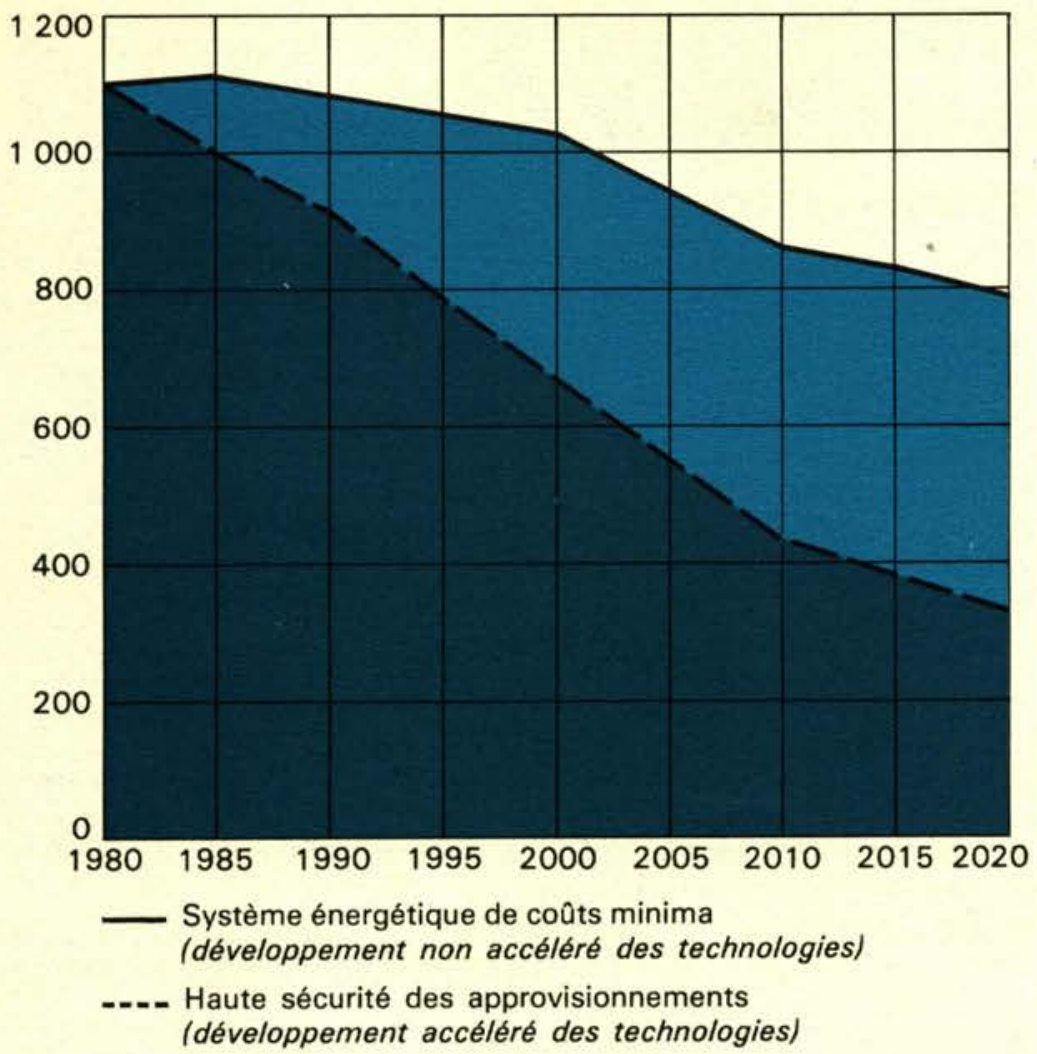

internationales, basées au laboratoire de Brookhaven, États-Unis, et au Centre de recherche nucléaire de Jülich, Allemagne. Le travail le plus long a été de déterminer les caractérisations économiques et techniques de presque toutes les nouvelles technologies énergétiques importantes ainsi que des systèmes énergétiques des quinze pays membres de l'AIE qui ont pris part au projet.

L'analyse des systèmes a été effectuée sur la base des besoins de chaque pays. Toutefois, l'évaluation ultérieure et l'estimation stratégique portent sur les besoins et le potentiel technologiques des pays de I'AIE en tant que groupe.

L'analyse se fonde sur les hypothèses-clefs suivantes:

- taux de croissance économiques modestes et en diminution progressive

- croissance continue des prix réels du pétrole et des autres formes d'énergie

- croissance considérablement réduite des besoins énergétiques par suite d'une utilisation plus efficace de l'énergie.

\section{Le scénario}

L'analyse a permis d'étudier divers scénarios liés aux oriententations adoptées par les pouvoirs publics (graphique). - L'un des scénarios de base suppose que la première priorité consistera à obtenir de l'énergie au coût le plus faible possible et que le rythme actuel de développement et de mise en place de la technologie ne sera pas modifié.

- Le deuxième scénario suppose que la priorité sera accordée à la diminution des importations pétrolières moyennant l'attribution d'une prime de coût pour les nouvelles technologies énergétiques permettant de remplacer le pétrole, et que le rythme de développement et de mise en place des nouvelles technologies de production et d'économies d'énergie sera accéléré.

- Diverses variantes ont permis d'analyser les conséquences de restrictions arbitraires apportées aux technologies de production de l'énergie, comme par exemple une limitation de l'emploi de l'énergie nucléaire ou du charbon.

Ces analyses sont intéressantes en ce qu'elles permettent d'étudier les phénomènes complexes de concurrence entre par exemple les carburants synthétiques et l'électricité, ou entre les technologies d'économies d'énergie, qui rendent possible une utilisation plus efficace de l'énergie, et les technologies de production d'énergie.

Cette méthode présente une caractéristique très importante du point de vue du personnel de la R-D, car elle permet d'estimer le potentiel relatif des diverses technologies énergétiques nouvelles et comment un accroissement des effort de R-D pourrait avancer la date à laquelle il sera possible d'en disposer.

Comme dans toute analyse numérique de ce type, les résultats ne sont significatifs que si les données utilisées sont précises. L'influence des variations de certaines hypothèses a été vérifiée, mais il n'a pas été possible d'étudier toutes les combinaisons d'événements futurs. Par conséquent, l'emploi des résultats détaillés nécessite une connaissance approfondie des hypothèses de base. La stratégie pour le groupe AIE doit tenir compte de ces incertitudes, ce que, effectivement, elle fait.

\section{Les conclusions}

L'analyse fait cependant ressortir un certain nombre de conclusions générales qui, en grande partie, sont indépendantes de la précision des hypothèses détaillées.

II est possible de réduire les quantités de pétrole importé de type classique au cours de la période $1980-2020$. Jusqu'à l'an 2000, la diminution provient essentiellement des économies d'énergie et de la généralisation des techniques existantes. Les nouvelles techniques des combustibles liquides pourront commencer à jouer un rôle notable entre l'an 2000 et 2020 . Mais, au rythme actuel des efforts de $R-D$ et $D$ et de commercialisation en matière d'énergie, les réductions prévues des importations pétrolières s'effectuent très progressivement et les pays de I'AIE continueraient à rester très vulnérables aux interruptions d'approvisionnement.

Sauf si la mise en place des nouvelles techniques est accélérée, celles-ci ne peuvent pas contribuer avant l'an 2000 à réduire de beaucoup plus la demande pétrolière. Avec une accélération de leur développement la réduction est importante.

Les nouvelles techniques d'économies d'énergie peuvent jouer un rôle très important au cours des vingt prochaines années et aider I'AIE à atteindre ses objectifs. La contribution de ces techniques peut d'ailleurs, dans certains pays, dépasser celle des techniques de production d'énergie.

Dans presque tous les pays de l'AIE, l'augmentation de la production d'énergie primaire provient essentiellement du charbon et de l'électronucléaire. II faut donc accorder une priorité élevée aux efforts de R-D et $D$ qui contribuent à permettre ou à faciliter l'emploi de ces sources (par exemple, technologies de protection de I'environnement en cas d'utilisation du charbon, sûreté nucléaire et gestion des déchets radioactifs).

La croissance du secteur "électricité" reste supérieure aux taux de croissance totale de la consommation d'énergie dans tous les cas étudiés, mais diminue dans chaque tranche successive de dix ans. II faudra consacrer des efforts de R-D suffisants aux techniques de production d'électricité, mais certains pays devront mettre plus spécialement l'accent sur la production d'énergie non électrique.

Certaines techniques de production d'énergies renouvelables commenceront à jouer un rôle notable avant l'an 2000. Cependant, même si les efforts pour mettre en place de telles techniques sont accélérés, les sources non renouvelables devront couvrir la majeure partie des besoins. En conséquence, les efforts de $R-D$ et $D$ devraient être concentrés sur les techniques les plus prometteuses de production d'énergie renouvelable, plutôt que de leur accorder une place égale.

L'analyse montre qu'une limitation de l'accroissement de l'emploi des combustibles fossiles autres que le pétrole aurait des répercussions très considérables sur les économies ainsi que sur 


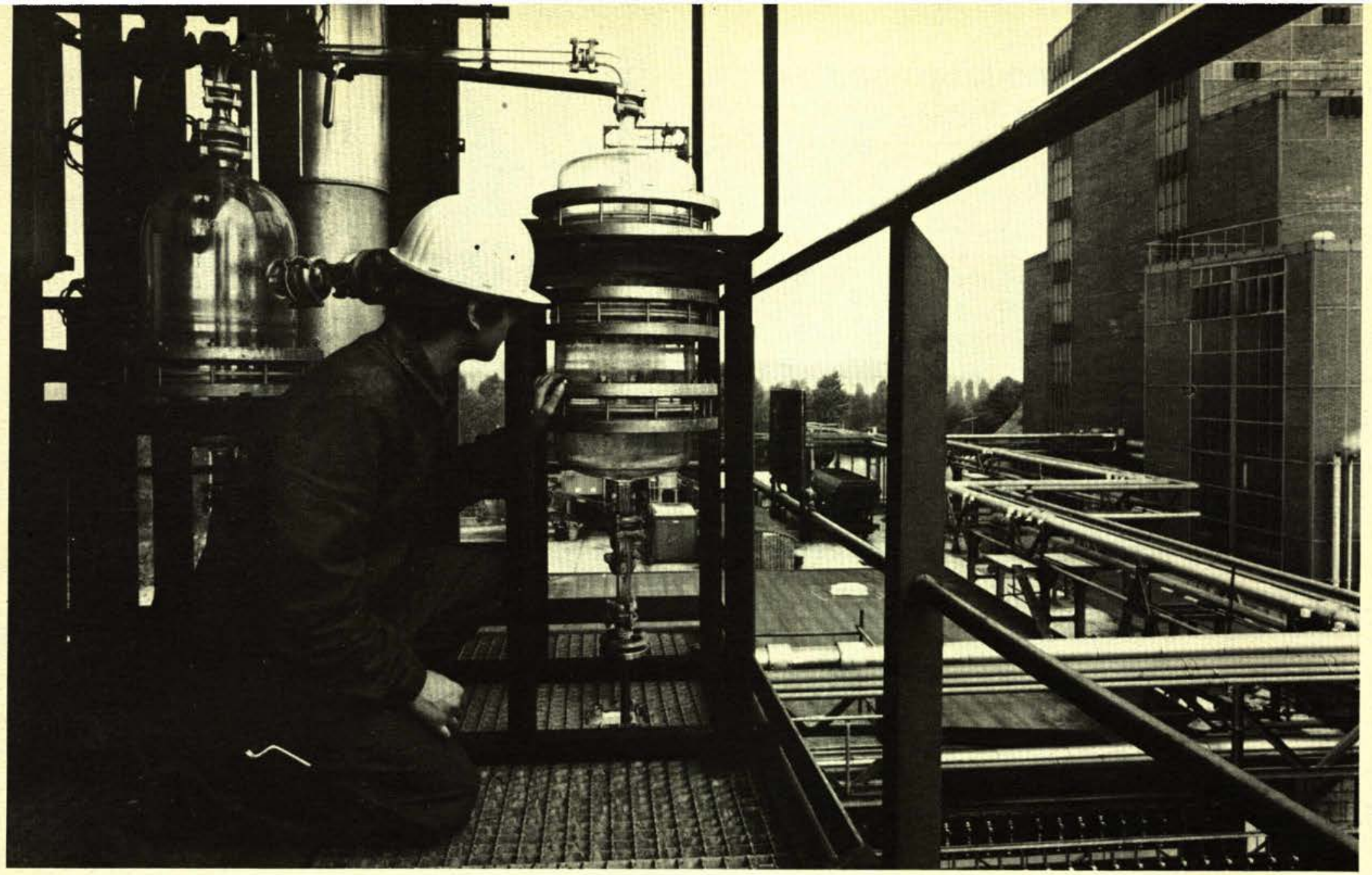

Gazéification de charbon en Allemagne.

les coâts de l'énergie, et provoquerait une augmentation des importations de pétrole et de gaz naturel. Ce scénario aboutirait à une situation plus grave que celles découlant de tous les autres scénarios utilisés; des programmes dynamiques de $R-D$ et $D$ axés sur les techniques de lutte contre la pollution de l'environnement en cas d'utilisation du charbon devraient donc être mis en œuvre pour empêcher cette situation de se matérialiser.

L'analyse du scénario fondé sur l'hypothèse d'une limitation de la croissance de l'électronucléaire montre qu'il ne suffirait pas de quelques techniques de remplacement mais qu'il faudrait faire appel à une large gamme de technologies plus coQteuses. La R-D et D sur la sûreté nucléaire et sur l'extrémité aval du cycle du combustible nucléaire devrait faire l'objet d'efforts soutenus pour permettre une extension des techniques de l'électronucléaire.

\section{Dans quelle mesure sont-elles réalistes ?}

Ces conclusions sont-elles optimistes ou pessimistes? Les données utilisées ont été préparées en faisant appel à l'avis de spécialistes les mieux qualifiés mais, à certains égards, cette méthode suppose des circonstances idéales. II serait donc prudent de penser que des contraintes techniques, économiques et sociales pourraient être plus importantes que prévu. Voici les questions les plus préoccupantes que cette analyse a soulevé.

- Est-il possible de tripler l'extraction et l'utilisation du charbon au cours des trente années à venir?

- La production d'électricité d'origine nucléaire peut-elle être accrue jusqu'au point indiqué comme souhaitable par les résultats de l'analyse?

- Est-il possible de surmonter les obstacles à la commercialisation de façon que les nouvelles technologies soient mises en place aux dates et à la cadence indiquées dans les résultats?

- En conséquence, est-il possible de réduire à l'avenir, et dans la proportion indiquée par les résultats, la demande de pétrole importé?
La réponse à ces questions dépasse le cadre du projet. II faut toutefois noter qu'elles reflètent l'inquiétude que les sources d'énergies classiques pourraient ne pas fournir la part qui leur est fixée par les résultats de l'analyse. Par conséquent, si les pays de l'AIE veulent éviter les conséquences économiques graves d'une désorganisation de leurs approvisionnements en énergie, les nouvelles technologies doivent être mises au point et appliquées avec une urgence accrue.

\section{La stratégie de l'AIE en tant que groupe}

La stratégie pour le groupe AIE a pour objet de soutenir une orientation de l'action visant à un haut degré de sécurité des approvisionnements énergétiques et se traduisant par une réduction considérable des importations de pétrole de l'ensemble des pays membres de l'Agence, sans pour autant entraîner des couts supplémentaires prohibitifs. La stratégie pour le groupe ne cherche pas à s'identifier à chaque stratégie nationale de $R-D$ et $D$; elle doit servir de schéma directeur au programme de $R-D$ et $D$ des différents pays membres et leur permettre de tenir compte des besoins collectifs de l'AIE. Elle porte sur quatre points.

- Elle classe toutes les nouvelles technologies prometteuse des divers domaines en quatre catégories de priorité, la première priorité étant affectée à celles qui devraient permettre de produire les plus grandes quantités d'énergie, notamment sous une forme qui se substitue au pétrole, au cout le plus bas compatible avec un haut degré de sécurité (à notre connaissance, c'est la première fois qu'un groupe de pays a déterminé d'un commun accord les priorités à accorder aux technologies énergétiques).

- Elle indique les actions à mener en vue des prochaines étapes de chaque domaine technologique.

- Elle recommande d'accélérer le rythme de mise au point et d'utilisation des technologies énergétiques pour permettre de réduire considérablement les importations pétrolières.

- Elle souligne l'importance des problèmes d'environnement à chaque étape de la mise au point des technologies en insistant 
STRATÉGIE ÉNERGÉTIQUE DU GROUPE AIE SUR LA R-D et D

\section{PRIORITÉS}

\section{A ACCORDER AUX DIVERSES TECHNOLOGIES(1)}

\section{PRIORITÉ N ${ }^{\circ} 1$}

\section{Utilisation finale}

Économies d'énergie - systèmes de transport automobile

Économies d'énergie - équipements utilisés dans la construction (surtout pompes à chaleur) Économies d'énergie dans l'industrie Chauffage et climatisation solaires pour le secteur résidentiel et commercial

\section{Production}

Récupération assistée du gaz

Récupération assistée du pétrole

Sables asphaltiques et schistes bitumineux

Transformation

Réacteurs convertisseurs nucléaires avancés Combustibles de remplacement pour les transports

Réacteurs surrégénérateurs

Liquéfaction du charbon

Technologies d'appoint essentielles

permettant d'utiliser au mieux les

systèmes énergétiques existants

Technologies du charbon qui assurent une protection de l'environnement (y compris la combustion en lit fluidisé sous pression atmosphérique)

Extraction du charbon

Sûreté des réacteurs nucléaires

Cycle du combustible nucléaire

\section{PRIORITÉ Nº 2}

\section{Production}

Énergie géothermique (hydrothermique)

\section{Transformation}

Cycle combiné (y compris production de gaz à faible pouvoir calorifique

et combustion en lit fluidisé sous pression) Combustibles tirés de la biomasse

Production de gaz à pouvoir calorifique élevé

\section{PRIORITÉ N 3}

Utilisation finale

Automobile électrique

Production

Méthane géopressurisé

Énergie éolienne

\section{PRIORITÉ N॰ 4}

Production

Roches chaudes et sèches - énergie géothermique

Énergie des océans (marées, vagues, transformation de l'énergie thermique des océans)

Électricité d'origine solaire

Transformation

Piles à combustible

Fusion(2)

Production de gaz à pouvoir calorifique faible ou moyen

Énergie magnétohydrodynamique

Systèmes de production d'hydrogène sans utilisation de combustibles fossiles

Gazéification souterraine du charbon

(1) Ces priorités s'appliquent à l'ensemble du groupe des pays de l'AIE et non pas à chaque pays pris isolément.

(2) La fusion est classée en quatrième priorité parce qu'elle ne sera disponible que tardivement (2020). Toutefois, compte tenu de l'importante contribution qu'elle pourrait apporter et du long délai de mise en œuvre, il conviendrait d'y consacrer des efforts suffisants pour mettre au point la technologie correspondante.
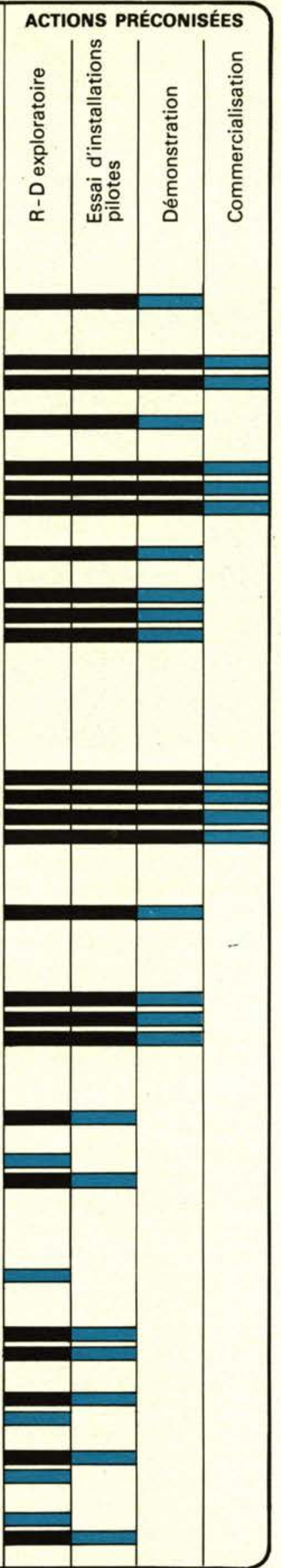


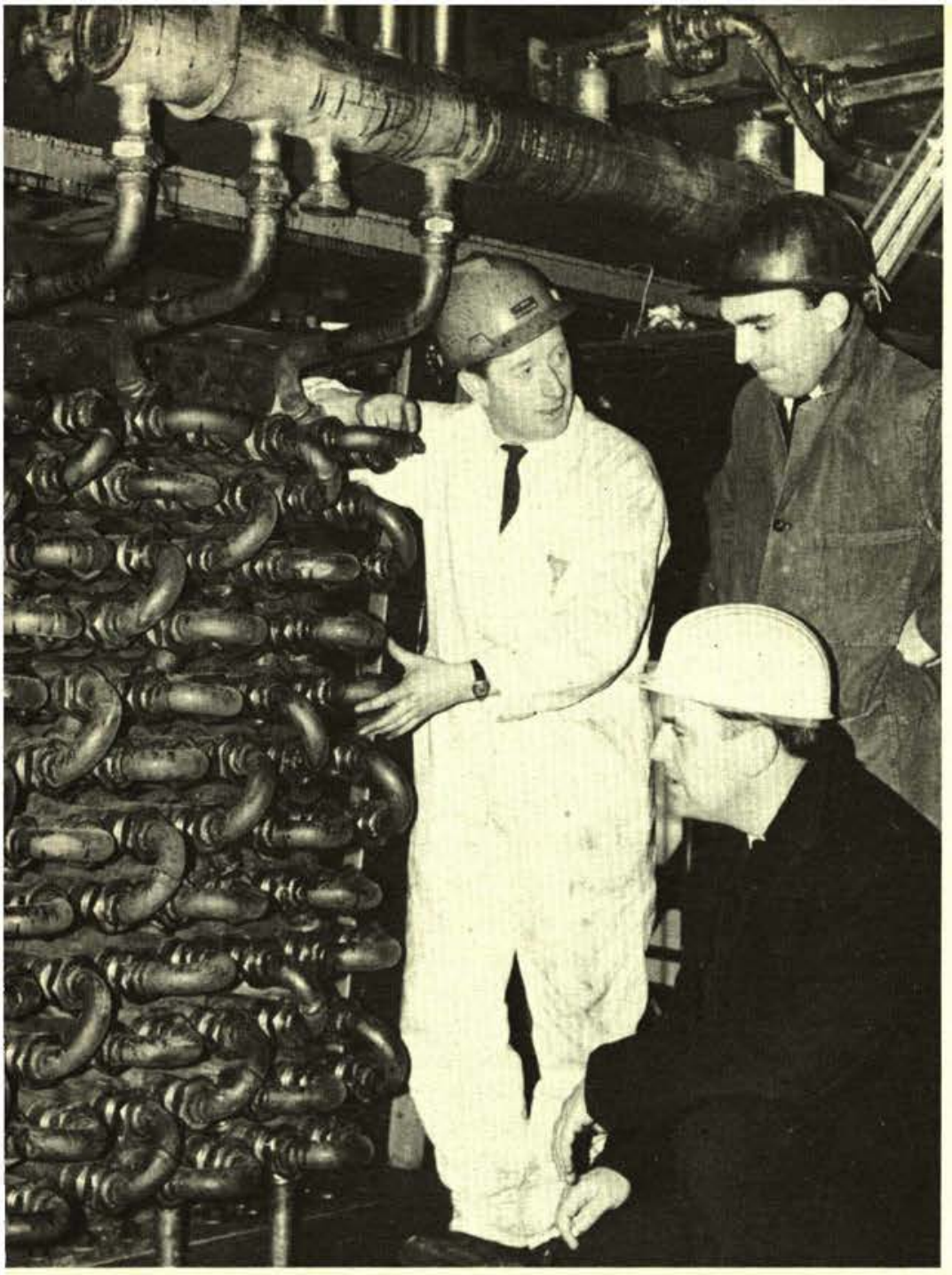

Chaudière à lit fluidisé en Grande-Bretagne.

ritaire qui n'en est qu'au début de la phase exploratoire peut, à juste titre, avoir un budget plus faible qu'une technologie moins prioritaire qui en est au stade de la démonstration. Si toutefois la technologie hautement prioritaire doit disposer de fonds supplémentaires pour concrétiser un progrès important et proche, le financement correspondant doit lui être accordé par priorité - les priorités s'appliquent aux pays de l'AIE pris dans leur ensemble ef non pas à chaque pays séparément.

\section{Actions préconisées}

La stratégie de développement technologique en matière d'énergie doit aussi indiquer les mesures à court terme qu'il conviendrait de prendre, compte tenu de l'état d'avancement et de l'importance potentielle de ces technologies. La stratégie précise donc les actions nécessaires ou possibles et les classe en quatre phases:

- opérations nécessaires pour arriver à l'utilisation commerciale dans un délai aussi bref que possible

- démonstrations à grande échelle

- expériences à échelle pilote

- R-D exploratoire.

Ces presciptions de la stratégie ont pour seul but d'indiquer la phase actuelle ou prochaine qu'il est justifié d'entreprendre. Dans chaque cas, il est parfois nécessaire de poursuivre les travaux de la phase précédente, qui englobe habituellement diverses opérations; la phase est alors désignée par l'indication du processus le plus avancé. L'état de développement indiqué n'est pas nécessairement atteint dans tous les pays, et chacun d'eux peut estimer qu'il ne lui convient pas de s'attaquer à certaines phases, ou même à aucune d'entre elles; certains pays peuvent ne pas travailler dans tel domaine, ou ne contribuer que pour partie à l'ensemble de l'effort nécessaire pour explorer et développer la technologie dont il s'agit.

\section{Accélération}

Les efforts des pays de I'AIE pour développer et mettre en service commercial usuel les nouvelles technologies énergétiques doivent être affectés d'une priorité notablement plus élevée que celle dont ils bénéficient à l'heure actuelle. Cela ne concerne pas seulement les dépenses des secteurs publics et privés pour la R-D et $D$ en matière d'énergie, mais s'applique également aux efforts considérables qui sont nécessaires pour surmonter les obstacles à la commercialisation des nouvelles technologies énergétiques.

L'analyse des systèmes montre bien la différence des résultats obtenus selon que la mise en place des nouvelles technologies énergétiques est accélérée ou non; si les orientations actuelles ne sont pas modifiées, les pays de l'AIE pourraient être obligés d'importer près de $60 \%$ de leur énergie sous forme de pétrole en l'an 2000 , alors que cette proportion pourrait être ramenée à $45 \%$ en cas d'accélération du développement et de la commercialisation des nouvelles technologies. II faut comparer la valeur de ces avantages aux coûts des programmes accélérés de $R-D$, coûts qui n'ont pas encore été estimés. Chaque pays devrait évaluer cas par cas les coûts et avantages de cette accélération.

\section{Environnement}

Presque toutes les technologies énergétiques affectent d'une manière ou d'une autre l'environnement. Les programmes de développement des nouvelles technologies énergétiques doivent comprendre l'étude des effets sur l'environnement, dont il doit être tenu soigneusement compte à toutes les phases du développement et avant la mise en place.

\section{Problèmes non technologiques liés à l'utilisation des nouveaux systèmes énergétiques}

Même lorsque les nouvelles technologies ont été mises au point avec succès, leur mise en place à la cadence voulue peut être affectée par toute une gamme de facteurs non technologiques, dont les plus importants suivent.

- Fixation rationnelle des prix. La mise en place des technologies nouvelles peut être retardée par un abaissement artificiel du prix des différents types d'énergie classique, résultant par exemple, d'un contrôle sélectif des prix.

- Orientations du commerce extérieur. II convient de faciliter les échanges d'énergie primaire ainsi que ceux portant sur les technologies et le savoir-faire en matière d'énergie.

- Incidences sociales. Les gouvernements devront établir des prévisions et des plans pour surmonter les éventuelles incidences sociales gênantes qui peuvent résulter d'une mise en place à grande échelle de certaines technologies énergétiques nouvelles. - Disponibilité des ressources annexes. Certaines ressources comme la main-d'œuvre qualifiée, les matières premières, l'infrastructure, la possiblité de disposer du sol et de l'eau, peuvent nécessiter des dispositions spéciales. Ce facteur est particulièrement important en cas d'utilisation accrue du charbon.

- Cadre réglementaire et structure industrielle. II est fréquent que la réglementation officielle et la structure industrielle aient été conçues en fonction de systèmes classiques et ne soient pas adaptées au caractère des nouvelles technologies.

$$
\text { *** }
$$

La réunion au niveau ministériel du Conseil de direction de l'AIE qui s'est tenue en mai 1980, a approuvé cette stratégie et a chargé le Comité pour la recherche et le développement en matière d'énergie de suivre de près et d'examiner périodiquement l'ensemble des efforts nationaux en matière de $R-D$ et $D$ pour voir s'ils correspondent bien à la stratégie pour le groupe. Au niveau national, chaque pays s'inspirera de la stratégie pour réaliser sa planification en matière de R-D et $D$ énergétique et pour fixer les ordres de priorité. 


\section{Diagnostic et analyse d'un problème tenace LE CHÔMAGE DES JEUNES}

e "Diagnostic révisé» du Secrétariat sur l'ampleur et les caractéristiques des problèmes posés par l'emploi et le chômage des jeunes (16-25 ans) mettent clairement en évidence la gravité de la situation actuelle et des perspectives à court terme. II existe peu de pays Membres - l'Allemagne, l'Autriche, le Danemark, le Japon, le Luxembourg et la Suisse - où le chômage des jeunes ne se présente pas comme un phénomène sérieux. Depuis la Conférence de l'OCDE à haut niveau de 1977 qui traitait spécifiquement de cette question, peu ou point de progrès ont été réalisés.

A très peu d'exceptions près, dont l'Allemagne, les États-Unis et le Royaume-Uni sont les exemples les plus notables, le taux de chômage des jeunes n'a cessé d'augmenter, passant pour l'ensemble de la zone OCDE (Turquie exclue) de 10,3 à $11,3 \%$. Et cela à un moment où le taux global a accusé une légère baisse (de 5,4 à $5,1 \%$ ). La part des jeunes dans le chômage total a par conséquent augmenté, passant de 44 à $47 \%$. Quant à la durée moyenne du chômage des 16-25 ans, elle s'est allongée dans pratiquement tous les pays, dont on dispose de données, de même que s'est accrue la proportion du chômage de longue durée pour l'ensemble des jeunes chômeurs. On note par ailleurs dans plusieurs pays Membres une détérioration de la situation des jeunes adultes (20-24 ans) par rapport à celle des adolescents (1620 ans) et de la main-d'œuvre féminine par rapport à la main-d'œuvre masculine dans les deux groupes d'âge.

De nombreuses recherches ont été entreprises en vue d'arriver à une meilleure compréhension des trois causes principales qui, de l'avis général, sont à l'origine du chômage des jeunes.

- La faiblesse des taux de croissance économique a évidemment réduit la demande de main-d'œuvre dont notamment la main-d'œuvre jeune.

- Des facteurs démographiques ont accentué le problème, la génération très nombreuse d'après-guerre étant arrivé sur le marché du travail au milieu des années 1970 .

- Les facteurs structure/s ont également leur importance. Le premier d'entre eux est la faiblesse de la position concurrentielle des jeunes sur le marché du travail qui reflète naturellement tout d'abord leur manque d'expérience. En outre les travailleurs plus âgés ont tendance à être protégés, souvent au détriment des jeunes, par les législations prévues pour la sécurité de l'emploi, les conventions collectives et les pratiques traditionnelles des entreprises en matière de gestion du personnel.

Un autre facteur souvent évoqué est l'évolution des coûts salariaux relatifs qui aurait contribué en partie au chômage des jeunes dans certains pays Membres. II convient cependant de souligner que cette thèse est très fragile et que l'on ne peut pas tirer des conclusions des données dont on dispose actuellement.

Par ailleurs, on ne doit pas perdre de vue l'importance pour les entreprises de toute la gamme des coûts non salariaux cotisations d'assurance-chômage, d'assurance-maladie et autres cotisations de sécurité sociale, cotisations aux caisses de retraite, frais de formation, avantages accessoires, comme les congés payés, etc. Dans la mesure où, comme c'est le cas dans bon nombre de pays Membres, les cotisations de sécurité sociale sont pla- fonnées, elles représentent une proportion d'autant plus importante des couts totaux de main-d'œuvre que le niveau des rémunérations est plus faible, ce qui est généralement le cas pour les jeunes.

Dernier facteur structurel: il se peut qu'un changement soit intervenu au niveau des aspirations (voir encadré) des jeunes générations en matière de travail. Cette tendance, ajoutée à l'extension des programmes de revenus complémentaires et l'augmentation du nombre des familles à salaires multiples, a pu réduire l'attachement des jeunes à leur travail, surtout parmi les moins de vingt ans.

Si les points de vue divergent quant au rôle exact joué par chacune des trois causes principales - conjoncturelle, démographique, structurelle - du chômage des jeunes, la prolongation de la période de croissance médiocre a, de l'avis général, joué un rôle prépondérant. II est peu probable que la situation à cet égard s'améliore sensiblement au cours des cinq prochaines années. Par contre, dans certains pays, les facteurs démographiques limiteront l'ampleur du problème. En effet, dans la mesure où la proportion de jeunes dans la population en âge de travailler diminue, les difficultés en seront quelque peu atténuées. Toutefois, le moment où l'importance relative de la cohorte des jeunes commencera à décroître n'est pas le même partout, et dans certains pays Membres, ce groupe devrait continuer à augmenter au début des années quatrevingts, ce qui créera des difficultés supplémentaires.

\section{Recherches récentes}

Des travaux récents sur le fonctionnement du marché du travail des

\section{LE CHÔMAGE DES JEUNES DANS DIX PAYS DE L'OCDE - 1970-1979}

\section{pourcentage}
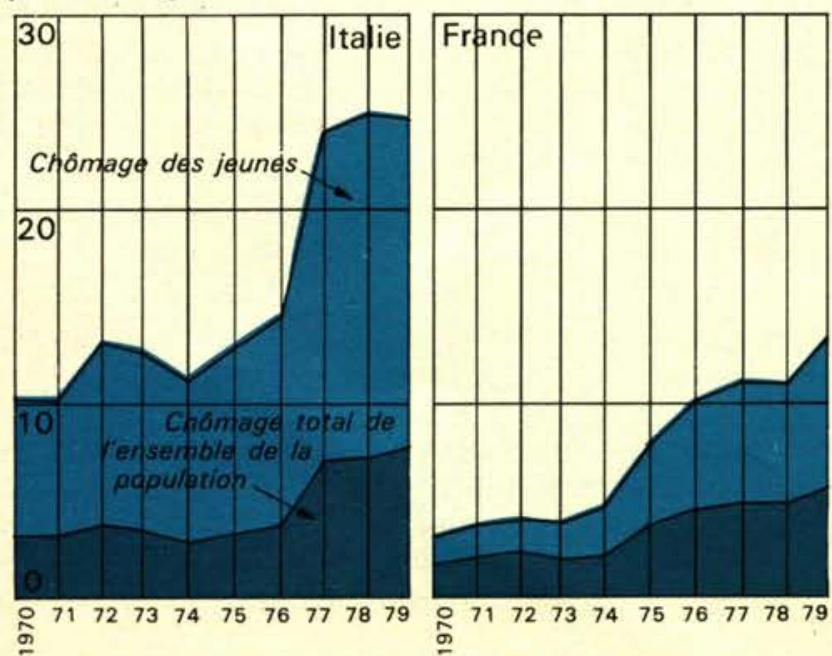
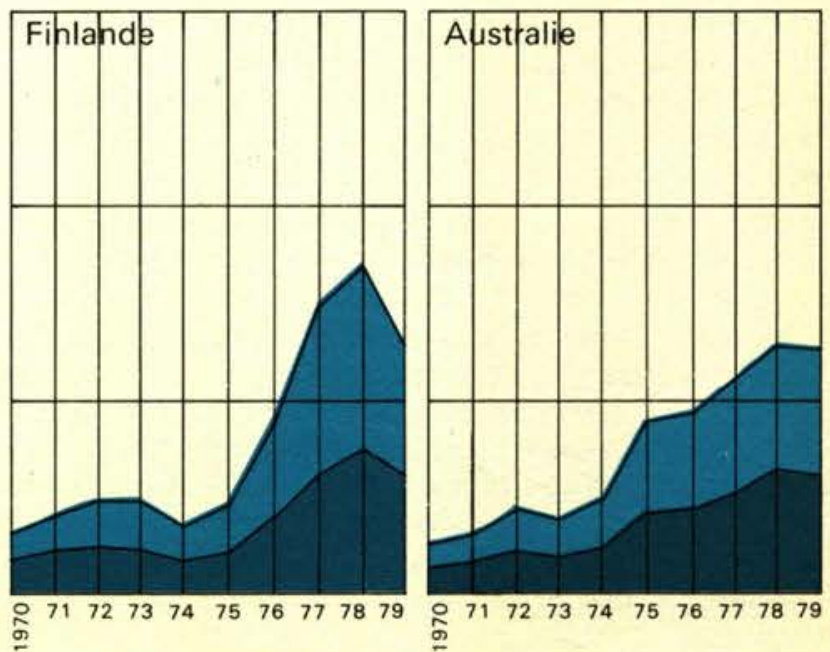
jeunes, notamment en Amérique du Nord, ont abouti à plusieurs constatations importantes. Si l'on ne sait pas avec certitude dans quelle mesure elles sont applicables aux autres pays Membres, un certain nombre d'indications fragmentaires donnent à penser que le schéma qu'elles esquissent est assez général pour mériter quelques développements.

\section{Taux de rotation élevés ...}

Diverses études montrent qu'un taux de rotation élevé est une caractéristique essentielle du comportement de la maind'œuvre jeune, surtout des moins de vingt ans. Un aspect remarquable de ces travaux est leur insistance, non seulement sur le passage de l'emploi au chômage, mais aussi sur le rôle que joue le fait d'entrer dans la population active et d'en sortir. C'est ainsi qu'une de ces études montre qu'en moyenne, au cours de chaque mois de la période allant de 1968 à 1976 , quelque $15 \%$ des hommes de moins de vingt ans faisant partie de la population active s'en étaient retirés, tandis que simultanément $21 \%$ de ceux qui n'en faisaient pas partie y étaient entrés. Avec l'âge, ces changements rapides diminuent de manière significative : la même étude montre qu'au cours d'un mois donné, la probabilité d'une renonciation à l'emploi est environ $60 \%$ moins grande pour un jeune homme adulte que pour un de moins de vingt ans.

La très grande fréquence des mouvements des jeunes entre les trois situations d'emploi, de chômage et de non-activité se traduit également par la durée très brève, en moyenne, des périodes qu'ils passent dans chacune d'entre elles. Chez les moins de vingt ans, la durée moyenne d'une période ininterrompue d'activité était dans les années 1968-76 d'environ six mois, contre quinze mois pour les jeunes adultes et soixante-six pour les hommes âgés de 25 à 59 ans. Cette brièveté vaut également pour les périodes de non-activité, dont la durée moyenne était de cinq mois pour les moins de vingt ans. En moyenne, la durée d'une période inin-

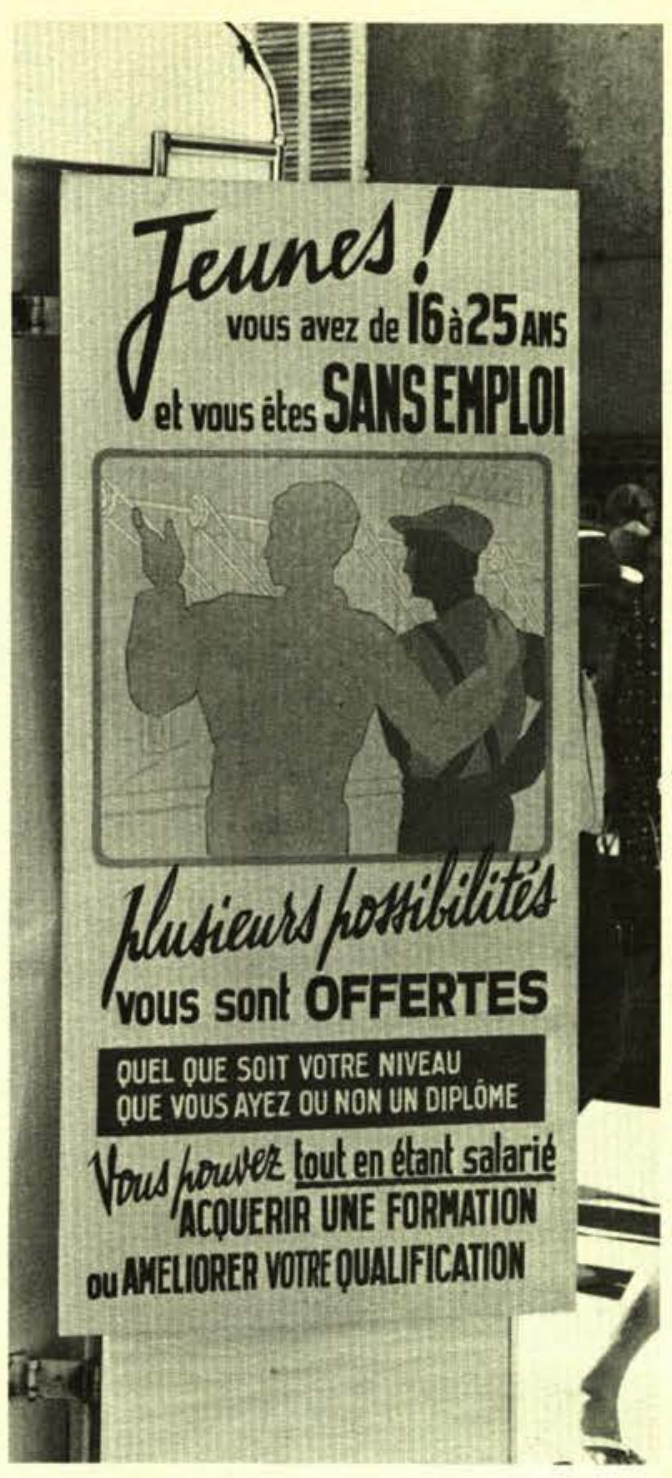

terrompue de chômage est également moindre pour les moins de vingt ans que pour les jeunes adultes; c'est ainsi qu'au cours des années 1968-76, elle était d'environ 1,7 mois pour les premiers, contre 2,3 pour les seconds. On peut donc penser que les fortes différences de taux de chômage entre les diverses classes d'âge correspondent essentiellement à des différences de taux de rotation.

La brièveté des périodes de chômage peut s'expliquer, soit par la facilité avec laquelle on trouve un nouvel emploi, soit par un taux élevé de sortie du marché du travail. De données relatives à 1974, il ressort que chez les garçons de moins de vingt ans, près de la moitié des périodes de chômage se sont terminées par leur sortie du marché du travail contre le quart seulement chez les jeunes adultes.

\section{... et leurs conséquences}

Les avis divergent quant aux conséquences de ces taux de rotation élevés. Les tenants de la "théorie du capital humain" soulignent les avantages que présente, pour l'amélioration du profil de carrière, un investissement précoce dans une formation et une expérience professionnelles continues. Si l'individu n'effectue pas cet investissement au cours des premières années de sa participation au marché du travail, du fait qu'il multiplie ses périodes de chômage, ses gains en seront réduits tout au long de sa vie:

"Les théories de la segmentation du marché du travail» confirmeraient également cette hypothèse de «l'effet traumatisant». Des périodes prolongées de chômage et d'inactivité risquent d'avoir des effets négatifs sur les habitudes de travail et l'expérience professionnelle, et de pousser l'individu vers le marché des emplois secondaires et instables.

Par contre, les modèles du marché du travail qui mettent l'accent sur la valeur de la recherche de l'emploi donnent à penser que quelques allées et venues sont à la fois souhaitables et nécessaires pour les moins de vingt ans. Le marché du travail des jeunes se caractérise en effet habituellement par de graves insuffisances de l'information, aussi bien côté employeurs que côté demandeurs d'emploi. Une période de recherche de travail pourrait donc constituer un investissement productif.

On ne dispose guère de données empiriques au sujet des "effets traumatisants". Une étude récente donne à penser qu'une longue période de chômage intervenant au cours des premières années peut accroître le risque pour l'intéressé de connaître le chômage par la suite. Toutefois, les résul-
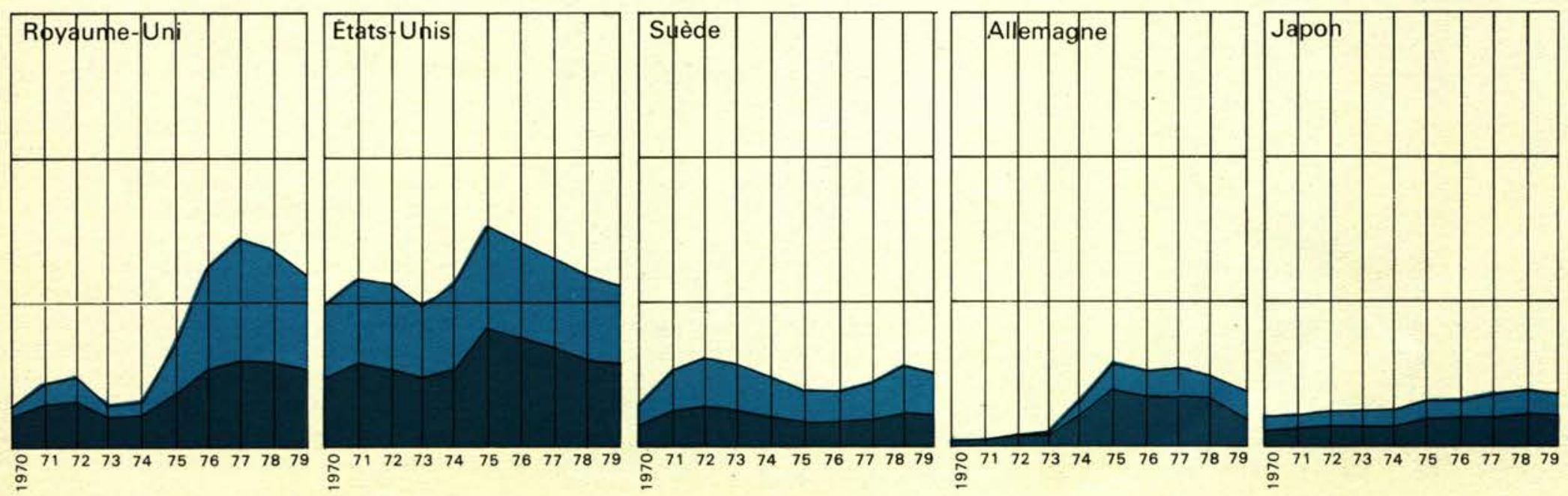


\section{LES ASPIRATIONS DES JEUNES}

U n point se dégage très nettement d'un projet de recherche sur l'attitude de la jeunesse récemment lancé par le CERI: l'attitude et les valeurs que l'on trouve chez les jeunes vis-à-vis de la vie active sont très diverses et semblent en corrélation avec des facteurs socio-économiques tels que le statut des parents, le niveau d'instruction, etc.

Jeunes non qualifiés. On considère systématiquement cette catégorie comme un objectif prioritaire de mesures spéciales. On justifie habituellement ce traitement du fait que cette catégorie est représentée de manière plus que proportionnelle parmi les chómeurs et que, chez les jeunes en question, un chómage prolongé pourrait avoir des conséquences graves pour la cohésion sociale.

Les évaluations effectuées au sujet des mesures prises en faveur de ces jeunes, ainsi que les recherches portant sur leur comportement «social $\|$, tendent à montrer qu'aussi bien les objectifs fixés à l'action que les craintes politiques qui s'expriment à leur sujet sont assez éloignés de la réalité. Loin d'étre systématiquement agités et furieux, ces jeunes peuvent plutôt être définis comme de plus en plus apathiques et coupés de leur environnement social; ils montrent peu d'intérét pour l'amélioration de leurs perspectives de carrière ou pour les efforts qui leur permettraient d'échapper aux emplois sans avenir que leur ouvre leur faible qualification. En outre, en dépit du manque de possibilités d'emploi intéressantes dans certaines régions, la plupart des jeunes issus de familles d'un bas niveau socio-économique refusent souvent de se déplacer de plus de quelques kilométres pour trouver un meilleur emploi.

D'une manière générale, les éléments dont on dispose donnent à penser que ces jeunes, au moment où ils abordent le marché du travail, sont déjà conditionnés pour profiter le moins possible des possibilités existantes. En d'autres termes, ces jeunes sont souvent si «réalistes „ quant à leurs perspectives d'emploi qu'ils en arrivent à nier d'avance toute possibilité de trouver du travail - prophétie qui ne manque pas dès lors de se vérifier.

Diverses sources corroborent l'opinion selon laquelle le probleme du chómage des jeunes les moins favorisés tient autant à leur manque de confiance en eux et d'esprit d'entreprise qu'à leurmanque de formation de base. «Le programme éducatif à l'intention des jeunes chómeurs „ mis en ceuvre en Australie a tiré les conclusions pratiques de cette hypothese: l'accent y est explicitement mis sur l'accroissement de la confiance en soi des jeunes qui y participent. De méme, une récente étude effectuée dans les pays nordiques montre combien il est important, pour le succès d'un programme consacré aux jeunes chómeurs, d'assurer une participation active de ces derniers à des situations de travail réelles.

II s'ensuit que les programmes visant les catégories défavorisées devraient se consacrer par priorité à renforcer leur confiance en soi. Il va sans dire que la meilleure façon d'atteindre ce but consiste à fournir aux jeunes qui ont définitivement tourné le dos à l'école et à l'instruction, ce dont ils ont le plus besoin, à savoir une expérience réelle du travail plutót qu'une formation en laboratoire.

Jeunes plus instruits. Si parmi les jeunes le taux de chómage tend habituellement à baisser à mesure que le niveau d'instruction s'élève, un nombre croissant de jeunes d'un niveau d'instruction supérieur à la moyenne subissent des périodes de chómage dans le cadre de la mauvaise situation que connaît actuellement le marché du travail. En fait, dans certains pays Membres (tels que I'Italie ou I'Espagne), on observe des taux de chómage très élevés parmi les jeunes diplómés de l'enseignement supérieur.

Jusqu'à présent, les responsables de l'action gouvernementale ne se sont guère préoccupés de mesures visant expressément à améliorer les perspectives d'emploi de ces jeunes. Or, certains signaux d'alarme peuvent donner à penser que, de tous les jeunes, c'est cette catégorie qui risque le plus d'avoir de "mauvaises devant la difficulté de trouver du travail et peut-étre d'adopter un comportement antisocial, tel que la délinquance ou l'abus de stupéfiants.

Dans l'ensemble, ces jeunes sont issus de classes sociales oú l'on est engagé à subordonner le gain immédiat au principe de la "récompense à retardement . Beaucoup d'entre eux ont supporté des années d'études et de retard dans la prise de responsabilité, avec comme perspective une promotion sociale. Si le chemin qu'ils suivaient n'était pas toujours facile, ni rectiligne, l'aboutissement en était généralement clair, prévisible et satisfaisant.

Cependant, ce processus risque d'être mis en cause par le niveau élevé du chómage des jeunes et la difficulté qui en résulte, pour des jeunes instruits, à trouver un emploi satisfaisant. Pour la première fois depuis des décennies les jeunes - surtout ceux qui sont issus des classes moyennes - affrontent de plus en plus le sentiment d'impuissance et d'absence de maitrise de leur existence qui de longue date sont le pain quotidien des catégories socio-économiques inférieures. Face à cette situation inhabituelle, source de tension, qu'aggrave encore l'impression que l'on ne fait rien pour résoudre les problèmes qui leur sont propres, ces jeunes risquent d'adopter de plus en plus un comportement social négatif. tats obtenus permettent également de penser que cet effet s'atténue assez rapidement. Quant au modèle qui met l'accent sur la valeur de la recherche de l'emploi, la brièveté des périodes de travail des moins de vingt ans a été invoquée pour mettre en doute que ce modele s'applique effectivement à cette classe d'âge.

Ces interprétations contradictoires aussi bien des causes que des conséquences des taux de rotation élevés dépendent de toute évidence beaucoup de la mesure dans laquelle on considère ces comportements comme volontaires ou involontaires. Ceux qui interprètent les observations effectuées comme l'indication d'un manque d'intérêt pour le travail au cours de cette période de l'existence soutiennent que le chômage précoce et les phases de non-activité peuvent correspondre à une consommation de loisirs, ou, en d'autres termes, que les moins de vingt ans souhaitent "traînailler» quelque peu avant de s'installer dans la vie professionnelle. D'autres, au contraire, estiment que le niveau élevé des taux de rotation reflète essentiellement le manque d'emplois. A l'appui de cette affirmation, ils font valoir que de nombreuses observations empiriques montrent la très forte influence exercée sur le chômage des jeunes par les fluctuations de la conjoncture.

\section{Un «noyau dur»}

Les taux de rotation élevés et les brèves périodes de chômage ininterrompues pourraient faire penser que les jeunes chômeurs souffrent peu de leur situation. En fait, ces constatations vont de pair avec celles qui indiquent l'existence d'un "noyau dur» de jeunes qui subissent de façon répétée des périodes de chômage prolongé.

Plusieurs études récentes indiquent clairement que la charge du chômage des jeunes est inégalement répartie : il existe un petit groupe qui rencontre de manière persistante de graves difficultés sur le marché du travail. Les résultats de l'enquête américaine annuelle de 1974 sur l'expérience professionnelle permettent de comparer la répartition des chômeurs et celle des semaines de chômage. Si quelque $70 \%$ des périodes de chômage subies par les garçons de moins de vingt ans durent moins d'un mois, plus de la moitié du total du chômage que connaît cette catégorie concerne ceux de ses membres $(8,4 \%$ de l'ensemble) qui ont été en chômage durant plus de six mois au cours de l'année. Ainsi, loin de se répartir également entre les jeunes, comme le donnerait à penser la brièveté moyenne des périodes de chômage consécutives, la charge du chômage est fortement concentrée. Les renseignements limités dont on dispose donnent à penser que la situation des membres de ce noyau dur ne s'améliore pas de manière significative lorsqu'ils passent dans la catégorie des 20 
à 24 ans. (On constate par ailleurs une concentration analogue parmi les adultes.)

A beaucoup d'égards, le marché du travail des jeunes aux États-Unis est très particulier. En premier lieu, les rapports entre l'école et la vie active sont très différents de ceux qui existent ailleurs; c'est ainsi qu'en $1976,30 \%$ des garçons et $26 \%$ des filles de moins de vingt ans poursuivaient des études à temps complet tout en travaillant. En second lieu, la concentration du chômage des moins de vingt ans se trouve largement au sein des minorités raciales et elle est en train de s'aggraver. Enfin, on ne saurait négliger l'évolution différente de la conjoncture depuis $1974 / 75$. Tandis que les États-Unis connaissaient une période de croissance économique soutenue, conduisant à la création d'un nombre d'emplois sans pré- cédent, l'économie de la plupart des autres pays de I'OCDE était languissante entraînant une augmentation de la durée moyenne des périodes de chômage interrompues pour les jeunes.

Les résultats obtenus par des recherches effectuées au Canada confirment l'observation sur le "noyau dur» faite aux États-Unis, selon laquelle une part importante de l'ensemble du chômage des jeunes (y compris les retraits du marché du travail) frappe ceux qui restent très longtemps sans emploi. C'est ainsi que l'enquête annuelle sur le comportement au travail montre qu'environ un quart des jeunes (15-24 ans) qui avaient été en chômage en 1977 ont subi plus de la moitié du total des mois de chômage que ces classes d'âge avaient supportés au cours de l'année. Cela pourrait signifier qu'une sous-catégorie de jeunes chômeurs est frappée de manière systématique et répétée, car celui qui, au cours d'une année, est resté longtemps sans emploi risque fort de se retrouver dans la même situation l'année suivante. Cette observation suggère à son tour que l'on pourrait peut-être, comme aux États-Unis, attribuer l'existence d'un grave chômage parmi les jeunes à certaines caractéristiques de leur personnalité ou de leur milieu. De fait, l'analyse effectuée au Canada donne à penser que le «chômage aggravé " présente une corrélation systématique et significative avec le manque d'instruction, la difficulté à passer de l'école au monde du travail (obtention du premier emploi), et le fait d'habiter une région de fort chômage en dehors des grandes villes.

\section{LES JEUNES ETRANGERS ET LE MONDE DU TRAVAIL}

Qui n'a pas sa petite idée sur la situation des jeunes étrangers sur le marché du travail? Faible niveau d'éducation, formation professionnelle inexistante ou insuffisante, métiers peu qualifiés, emploi instable, discrimination .....

Un rapport de l'OCDE(1) se propose de mesurer ce qui auparavant n'était que pressenti, d'établir le lien entre la réglementation et la réalité de façon à favoriser un aller-retour entre le droit et le fait.

Tâche ardue que celle de démontrer avec un outil très largement inadapté des phénomènes seulement perçus. Les statistiques existantes sont en effet soit conçues pour d'autres utilisations, soit tellement disparates que toute comparaison internationale devient difficile.

Par des recoupements entre diverses sources, des dépouillements minutieux, des discussions entre experts et en utilisant simultanément trois approches pour mener l'investigation - statistique, juridique, qualitative - la Direction des affaires sociales, de la main-d'œuvre et de l'éducation a réussi à établir un rapport novateur qui marque un progrès réel. I/ permet de mieux cerner certains aspects de la condition économique des jeunes étrangers de la deuxième génération(2), base essentielle pour agir en leur faveur.

L'article qui suit donne un bref aperçu des principales conclusions du rapport et esquisse ce qui reste à accomplir pour affiner ce nouvel instrument.

(1) II sera publié prochainement.

(2) Le rapport est fondé sur les travaux menés dans cinq pays : Allemagne, Belgique, France, Suede, Suisse.

\section{Une recherche statistique ...}

Dans les cinq pays étudiés, les jeunes étrangers âgés de moins de 25 ans sont au nombre d'environ 3800000 et représentent entre moins de $40 \%$ (France, Suisse) et près de $50 \%$ (Belgique) du total de la population étrangère résidante; par ailleurs, ils constituent entre 6,6\% (France) et 15,3\% (Belgique) de l'ensemble des jeunes (nationaux plus étrangers) appartenant à la même classe d'âge.

Près de 750000 d'entre eux sont actifs, soit de 14,3\% (France et Suisse) à 22,6\% (Belgique) du total des travailleurs étrangers et de $5,8 \%$ (France, Allemagne) à 9,9\% (Suisse) des actifs (nationaux plus étrangers) du même âge.

En 1978, les jeunes migrants représentaient entre $20 \%$ (France) et près de $33 \%$ (Suède) du total des étrangers à la recherche d'un emploi et entre $6,1 \%$ (France) et $15,9 \%$ (Belgique) des jeunes (nationaux et étrangers) dans la même situation.

Enfin, ils sont entre 5,6 (France) et $15,6 \%$ (Belgique enseignement francophone uniquement) dans l'ensemble des effectifs scolarisés dans l'enseignement secondaire.

Outre ce constat statistique, l'analyse met en relief de nombreuses caractéristiques dont notamment :

- la propension de plus en plus forte des étrangères à occuper un emploi; dans tous les pays étudiés, la part des immigrées actives dans l'ensemble de la main-d'œuvre étrangère est d'autant plus élevée que les classes d'âge sont plus jeunes

- dans tous les pays également, le taux d'activité des jeunes étrangers (les deux sexes réunis) est toujours inférieur à celui des jeunes nationaux alors qu'une situation inverse caractérise les travailleurs âgés de plus de 25 ans (graphique)

- une certaine reproduction de la force de travail étrangère en ce sens que les jeunes migrants se retrouvent au début de leur vie active en proportion aussi élevée que leurs pères dans quelques catégories professionnelles, celle d'ouvrier plus particulièrement - une très grande similitude entre l'évolution du chômage des jeunes (nationaux et étrangers) et celle du chômage des migrants de la seconde génération; en conséquence, chaque fois que la part des demandeurs d'emploi âgés de moins de 25 ans progresse ou diminue, celle des jeunes étrangers du même groupe d'âge à la recherche d'un emploi s'inscrit dans la même tendance - enfin, une présence des jeunes étrangers plus marquée que celle des jeunes nationaux du même âge dans les cycles d'ensei- 


\section{NATIONAUX ET ÉTRANGERS : TAUX D’ACTIVITÉ COMPARÉS PAR TRANCHE D’ÂGE pourcentage}

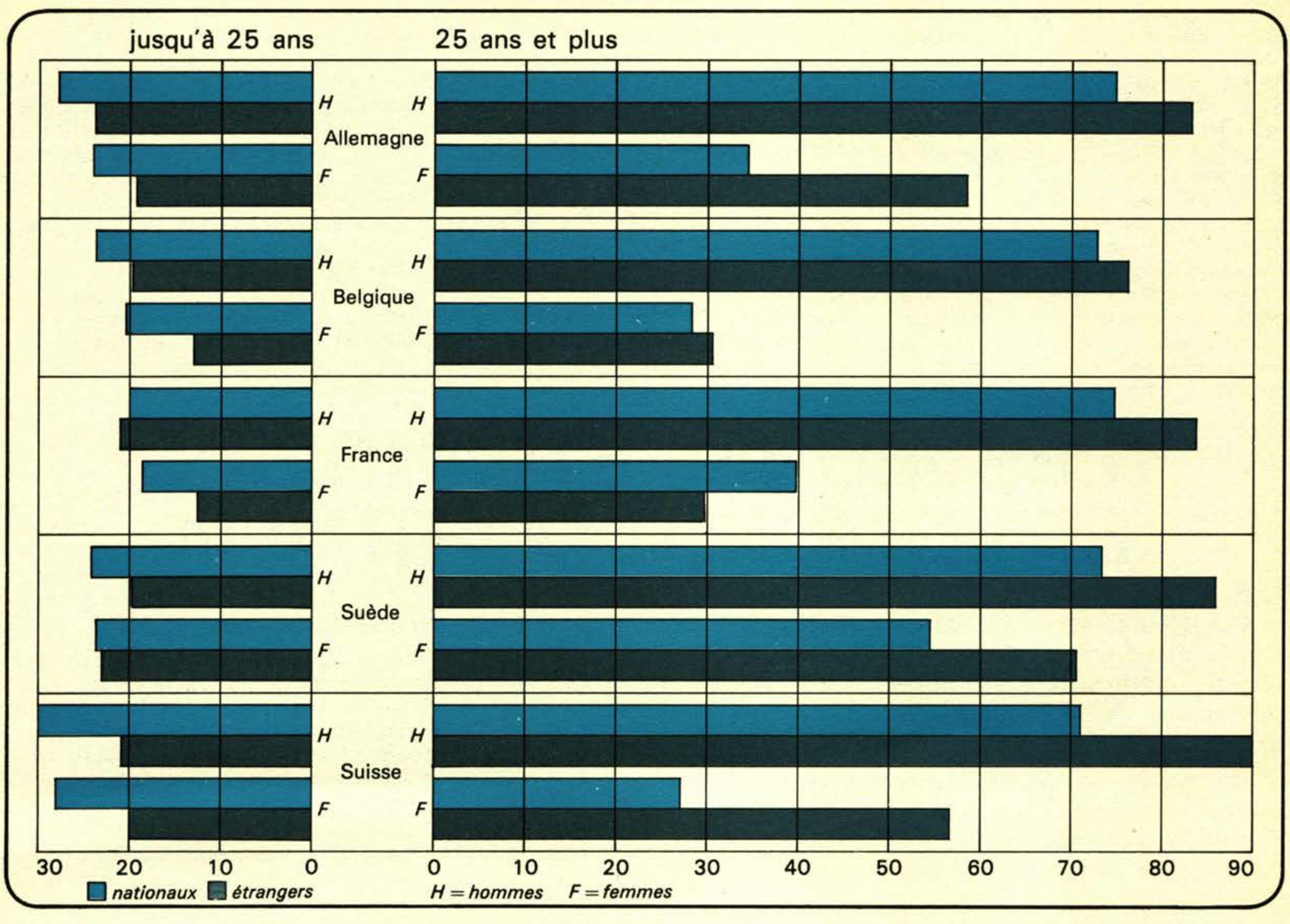

TOTAL DES HOMMES + FEMMES

pourcentage

\begin{tabular}{|l|c|c|c|c|c|c|c|c|c|c|}
\hline \multicolumn{1}{|c|}{ Tranche d'áge } & \multicolumn{2}{|c|}{ Allemagne } & \multicolumn{2}{c|}{ Belgique } & \multicolumn{2}{c|}{ France } & \multicolumn{2}{c|}{ Suède } & \multicolumn{2}{c|}{ Suisse } \\
\hline Jusqu'à 25 ans & 26,0 & 21,5 & 22,0 & 16,3 & 19,3 & 16,8 & 23,8 & 21,4 & 29,0 & 20,4 \\
\hline 25 ans et au-dessus & 52,4 & 73,8 & 49,0 & 55,5 & 54,8 & 64,6 & 63,5 & 78,8 & 47,4 & 75,7 \\
\hline
\end{tabular}

\section{$\square$ nationaux $\square$ étrangers}

gnement les plus courts et dans les actions de formation préparant à des métiers où le taux d'implantation étrangère est déjà élevé, et, dans le cas des jeunes filles notamment, sans correspondance avec les besoins d'une société industrialisée.

\section{... juridique ...}

L'examen de la réglementation relative au séjour et à l'emploi des jeunes migrants a permis de constater les faits suivants quant à leur situation juridique vis-à-vis de l'emploi :

- dans tous les pays étudiés, les jeunes étrangers venus au titre de l'immigration familiale ou nés dans le pays hôte ont, en droit, la possibilité d'accéder à l'emploi selon les modalités plus ou moins libérales qui, en règle générale, dépendent de la durée de leur séjour avant l'entrée sur le marché du travail et de la nature du titre détenu par le travailleur rejoint au moment où s'opère le regroupement de la famille

- des dispositions permettant un accès sans entrave à l'emploi ont été édictées dans les différents pays, mais il subsiste des cas où les jeunes migrants qui ne remplissent pas les conditions nécessaires pour en bénéficier, n'ont pas l'assurance de pouvoir exercer une activité à l'issue de leur scolarité ou de leur période de formation.

\section{... et qualitative}

La recherche bibliographique réalisée à partir des études effectuées sur le thème central du rapport dans les cinq pays a apporté à ce travail une connotation qualitative sur des faits qui échappent à toute appréhension statistique ou réglementaire. Cette prise en compte du vécu quotidien des migrants de la seconde génération est un complément utile aux autres types d'investigation.

Elle a permis dans un premier temps, de vérifier que les aspects dégagés par un examen des statistiques et des textes étaient corroborés par le résultat des travaux menés sur des échantillons et des aires géographiques plus limités. En un mot, l'approche micro-économique, dont on pouvait craindre le côté parcellaire et anecdotique, est venue étayer les conclusions de l'analyse macroéconomique, trop détachée, elle, de la réalité. Que ce soit en 


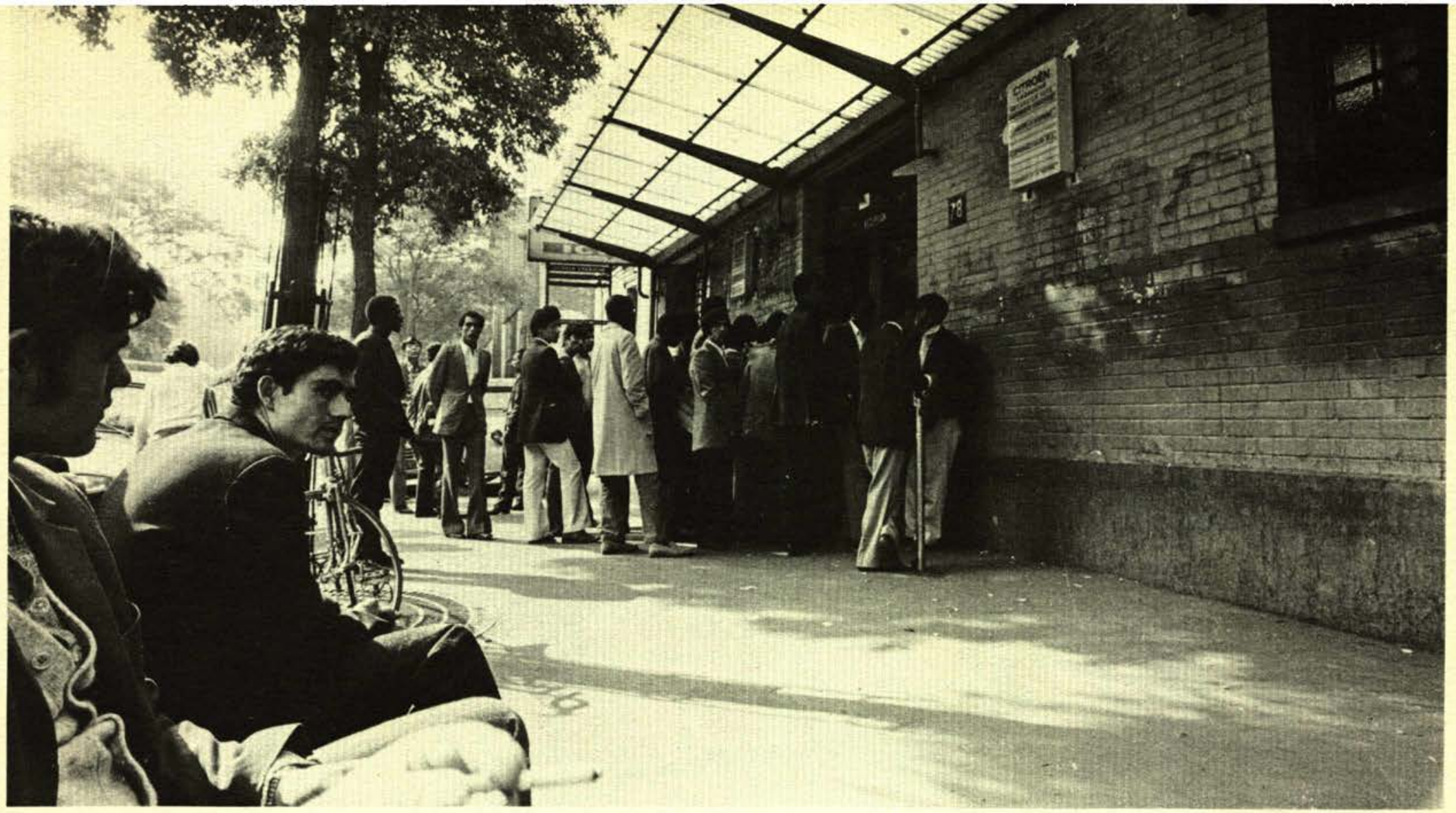

Les jeunes étrangers sont plus exposés au chómage que les nationaux.

\section{DE QUI S'AGIT-IL?}

Rien n'est plus imprécis que le terme «deuxième génération de migrants «. Pour plusieurs raisons:

- pour certaines nationalités, dont l'expatriation est antérieure à la dernière guerre mondiale, il s'agit plus de la troisième que de la deuxième génération

- les contours juridiques sont flous: un certain nombre d'enfants présentement considérés comme étrangers seront des nationaux dans quelques années (soit parce que compris dans l'effet collectif de la naturalisation de leurs parents, soit parce qu'ils auront réclamé, avant la majorité, le bénéfice de la procédure plus souple généralement instituée par les pays d'accueil pour les jeunes nés sur leur soll sans que, pour autant, leurs caractéristiques socio-économiques en soient profondément modifiées. Et même, dans certains cas, leurs parents les croient étrangers alors que le pays d'emploi les compte parmi ses nationaux lcas des jeunes Algériens nés en

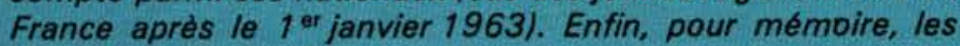
situations de double nationalité.

Cela n'est pas sans poser des problemes pour l'analyse. A double titre.

- une législation permettant une naturalisation rapide tend à "révacuer » hors de la catégorie cjeunes étrangers »-et donc à laisser en dehors du champ de l'étude - un nombre important d'individus qui, dans d'autres pays récepteurs, y demeureraient. C'est le cas de la Suede où, entre 1968 et 1978, 75500 jeunes de 0 d 24 ans ont été naturalisés (soit 45,6\% du total) parmi lesquels 46700 sont nés en Suède et 28800 à l'étranger

- un biais important peut résulter du rapprochement de deux séries statistiques, fondées l'une sur les déclarations des personnes elles-mémes (recensement, par exemple), l'autre sur un enregistrement par une autorité "rextérieure » à la famille : inscription à l'école, à l'agence pour l'emploi, etc....

Quitte à rester approximatif, la population analysée dans le rapport est celle des jeunes de moins de 25 ans, d'origine étrangère et qui n'ont pas formellement acquis la nationalité du pays d'accueil. matière de déroulement de la scolarité, d'absence de qualification, de propension à l'emploi, de type d'activité exercée ou de vulnérabilité au chômage, aucune dissonance n'est apparue entre les deux modes d'évaluation.

En second lieu, cela a permis de compléter le tour d'horizon en éclairant certains aspects qui se prêtent mal (ou pas du tout) à une mesure globale. Ainsi, les études monographiques ont mis en évidence le handicap majeur que constitue une médiocre connaissance de la langue du pays d'accueil non seulement au cours de la période de formation mais également au moment où s'effectue l'entrée dans la vie active, par suite de l'ignorance des filières à suivre, des voies à choisir. De même, ont pu être mieux saisies les conditions de travail - et la perception qu'en ont les intéressés eux-mêmes -, le comportement - réservé chez la plupart - visà-vis de la mobilité géographique, l'absence ou la faible mobilité sociale et l'attitude - variable suivant les pays - face à la participation syndicale et associative.

Pour compléter ces recherches et donc avoir une vue encore plus précise de la seconde génération, il faudrait étudier plus avant un certain nombre d'éléments : les flux (interne et externe) d'entrée sur le marché du travail, les rémunérations perçues, les modalités réglementaires d'accès à la formation professionnelle et aux services de l'emploi, l'indemnisation en cas de chômage.

Cependant, tel qu'il est, ce rapport présente un aspect novateur et constitue une base suffisamment étayée pour avancer certaines conclusions.

L'amélioration des appareils statistiques nationaux paraît être la condition première pour que l'on puisse affiner le diagnostic. C'est ainsi que l'absence fréquente de la variable "âge " a fait qu'on a do abandonner plusieurs pistes de recherche. A titre d'exemple, il n'a pas été possible de déterminer ni les types d'emploi, ni les qualifications professionnnelles de jeunes migrants entrant sur le marché du travail (à la suite d'une primo-immigration, phénomène désormais rare, mais surtout à un accès à l'emploi à la fin de la scolarité dans le pays d'accueil). Sur ce point, le recours aux statistiques de "stock" n'a été qu'un pis-aller. Cette même lacune a été rencontrée dans plusieurs autres domaines (chômage, formation professionnelle, etc.).

Par ailleurs, lorsque l'âge était pris en compte, la répartition par 


\section{TENDANCES}

\section{RECENTES DE L'AIDE}

\section{Volume}

\section{Aide publique au développement (APD) des pays du CAD}

En 1979, les versements nets d'aide publique au développement effectués par les membres du CAD se sont chiffrés à 22,3 milliards de dollars. Par rapport à 1978 , ils ont progressé en dollars courants de 2,3 milliards, soit de $11 \%$, ce qui après correction de la hausse des prix (1) correspond à une augmentation en termes réels de l'ordre de $2 \%$, légèrement au-dessous du taux d'accroissement tendanciel de ces dernières années.

Le PNB de l'ensemble des membres du CAD s'étant accru en dollars courants d'à peu près $13 \%$ par rapport à 1978 , le rap- port de leur APD totale à leur PNB global a légèrement fléchi en 1979, passant de $0,35 \%$ à $0,34 \%$.

Sur les dix-sept pays du CAD, douze ont accru leur APD par rapport à leur PNB, Toutefois, le montant en dollars des versements nets d'APD des États-Unis a sensiblement diminué, de sorte que le rapport de leur APD à leur PNB a fortement baissé (2). Le Canada, bien que son APD ait augmenté légèrement en monnaie nationale, a également enregistré une baisse de I'APD exprimée en dollars et en pourcentage du PNB. S'agissant de deux donneurs importants, la baisse de l'APD des États-Unis et, dans une moindre mesure, la stabilité relative du programme du Canada, a freiné l'accroissement de parfois dans une mesure très substantielle.

\section{A. APD EN POURCENTAGE DU PNB}

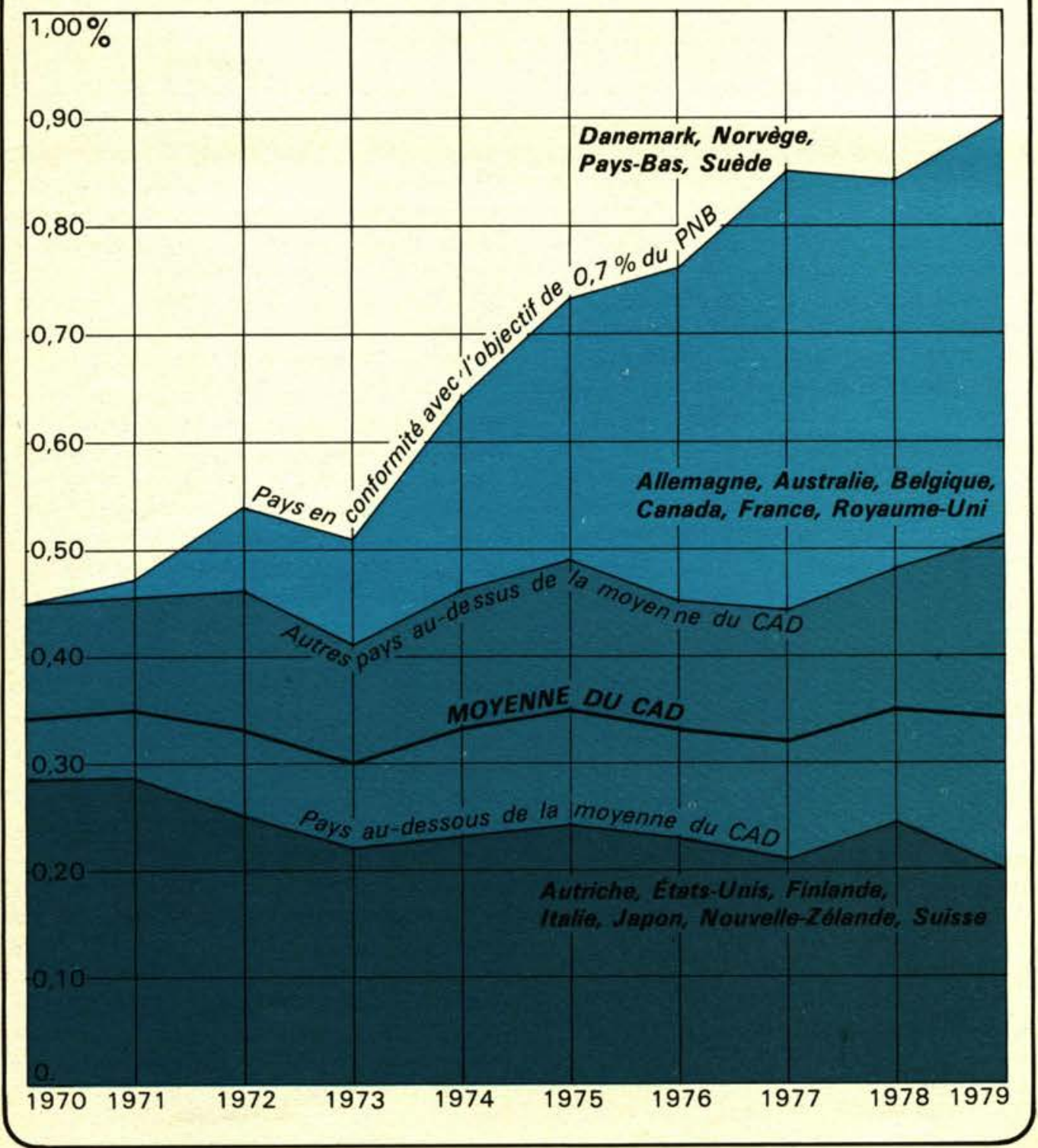

I'APD de l'ensemble des pays membres. Abstraction faite de ces deux pays, l'APD fournie par les autres pays du CAD pris collectivement est passée de $0,39 \%$ à $0,43 \%$ de leur PNB global, et son accroissement en termes réels est estimé à $15 \%$.

La situation inflationniste a conduit un certain nombre de pays du CAD à restreindre leurs dépenses publiques globales, ce qui risque de se traduire par un plafonnement, voire, dans un ou deux cas, une réduction de I'APD en termes réels. Toutefois, la plupart des membres ont affirmé leur intention d'élargir sensiblement leur programme d'APD et plusieurs d'entre eux mettent actuellement à exécution les plans qu'ils avaient annoncés antérieurement en vue d'accroître les crédits budgétaires affectés à l'aide : aussi espère-t-on que la tendance à un accroissement modéré en termes réels de l'APD dispensée par l'ensemble des Membres du CAD pourra se poursuivre.

\section{Évolution par pays (3)}

En 1979, la Suede où les crédits ouverts au titre de l'aide ont représenté $1 \%$ du PNB, a accru de $22 \%$ ses versements nets d'APD (tout particulièrement ses dons bilatéraux) qui ont atteint 0,94\% du PNB (contre $0,90 \%$ en 1978). Cela fait ainsi cinq années de suite que la Suède occupe, seule ou avec un autre membre, la première place parmi les pays du CAD. Les Pays-Bas, qui partagent le second rang avec la Norvège, ont porté en 1979 le rapport APD/PNB au niveau le plus élevé jamais enregistré, soit $0,93 \% \quad(0,82 \%$ en 1978). Dans leur plan actuel à moyen terme, les Pays-Bas se sont fixés pour but d'affecter un montant de ressources budgétaires de l'ordre de 1,20\% du PNB au financement du développement, en majeure partie sous forme d'APD. La Norvège a elle aussi établi un record avec un rapport APD/PNB de 0,93\%. Ses dons bilatéraux ont augmenté dans une mesure particulièrement importante en 1979. Le Danemark qui avait réalisé l'objectif de $0,70 \%$ en 1978 et était ainsi le quatrième pays du CAD à l'avoir atteint, a maintenu à $0,75 \%$ en 1979 le rapport APD/PNB.

Dans le cas de la France, la tendance à la baisse du rapport APD/PNB que l'on observait ces dernières années s'est renversée $(0,59 \%$ en 1979 contre $0,57 \%$ en 1978), du fait notamment d'une forte augmentation des contributions de ce pays à la CEE et d'un accroissement sensible de ses dons bilatéraux. L'APD fournie par la

(1) Mesurée par un indice implicite synthétique des prix du PNB de l'ensemble des pays membres du CAD.

(2) LAPD fournie par l'Australie, I'Autriche, la Nouvelle-Zélande a également diminué en pourcentage du PNB, mais son montant en dollars a augmenté.

(3) Les différents pays sont étudiés dans l'ordre de leur classement d'aprés la part de I'APD dans le PNB 1979. 


\section{B. APD : PERFORMANCE PAR PAYS \\ pourcentage du PNB}

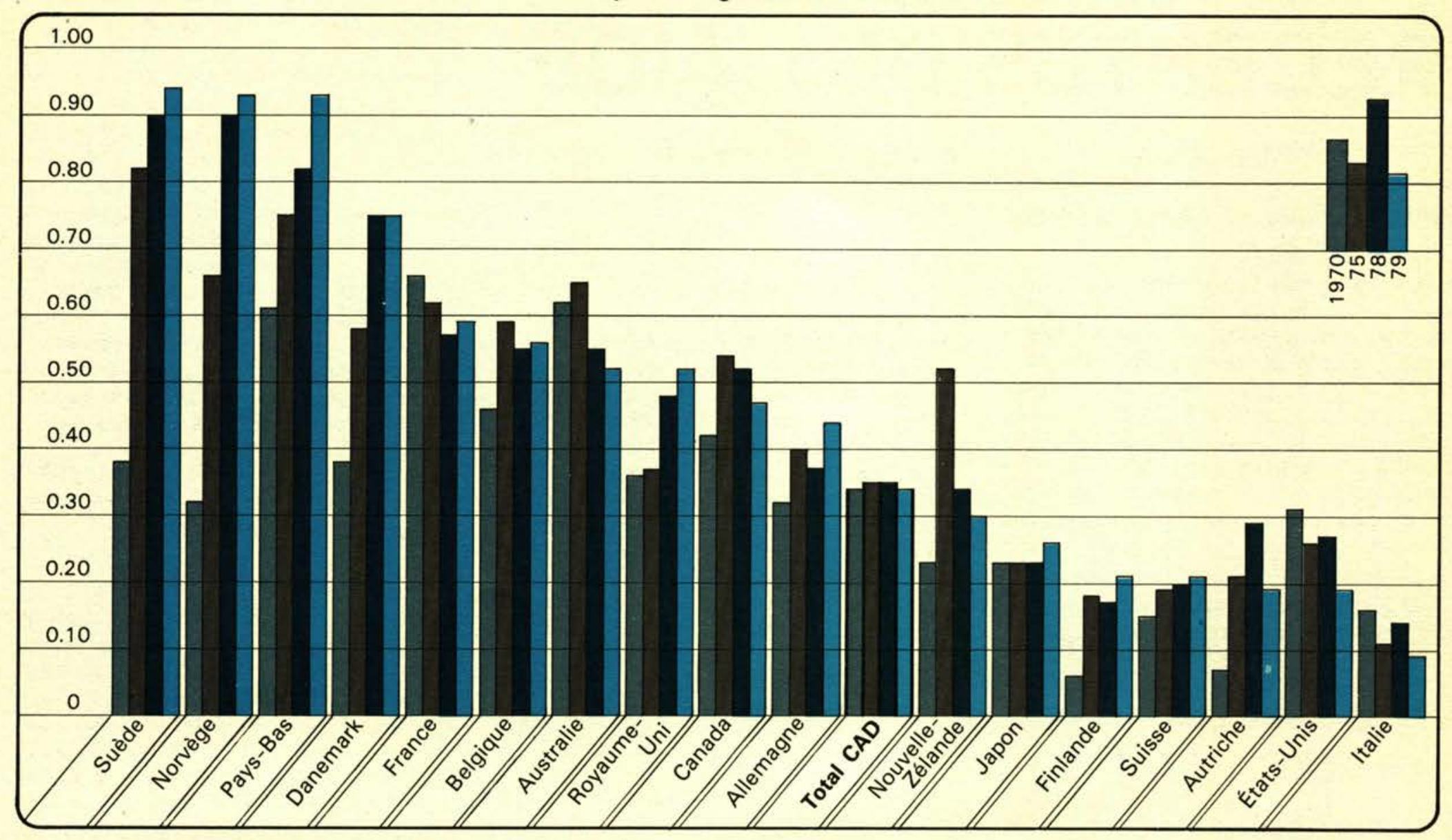

Belgique, qui a accru ses dons et ses prêts bilatéraux, a marqué un nouveau redressement en $1979-0,56 \%$ du PNB $(0,55 \%$ en 1978). Grâce à une progression spectaculaire de ses apports bilatéraux aussi bien que multilatéraux, le Royaume-Uni a porté le rapport APD/PNB à 0,52\%, ce qui est de loin son meilleur résultat depuis le début des années soixante. Ce rapport était de $0,37 \%$ en 1977 et de $0,48 \%$ en 1978. Toutefois, le Livre blanc sur les dépenses publiques paru récemment prévoit une réduction de l'aide extérieure du Royaume-Uni de l'ordre de $13 \%$ à prix constants en trois ans, de sorte que le chiffre de 1979 pourrait fort bien ne correspondre qu'à une pointe passagère. En dépit d'une augmentation de son montant en dollars, l'aide de l'Australie n'a représenté que 0,52 \% du PNB en 1979 au lieu de $0,55 \%$ en 1978 , ce fléchissement étant essentiellement du au profil temporel des émissions de billets à l'ordre des organismes multilatéraux, en particulier de I'IDA. Le montant en dollars des versements du Canada au titre de l'aide a diminué pour la première fois en dix ans, malgré une modeste augmentation en monnaie nationale, toutefois limitée en raison essentiellement de la politique budgétaire suivie depuis 1978. En pourcentage du PNB, I'APD du Canada est tombée de $0,52 \%$ en 1978 à $0,47 \%$ en 1979 .

Pour ce qui est du montant absolu en dollars de l'aide (3,4 milliards de dollars),
l'Allemagne est devenue en 1979 pratiquement exaequo avec la France, le second donneur du CAD (le donneur le plus important étant les États-Unis avec 4,6 milliards de dollars). Ses versements bilatéraux aussi bien que multilatéraux ont augmenté de façon notable et elle a fortement accru le rapport APD/PNB, qui est passé de $0,37 \%$ en 1978 à $0,44 \%$; d'après le plan actuel à moyen terme, le montant des crédits affectés au ministère de la coopération doit augmenter jusqu'en 1983 de $12 \frac{1}{2} \%$ par an, ou bien du double du pourcentage d'accroissement de l'ensemble du budget.

L'APD de la Nouvelle-Zélande, qui est restée à peu près inchangée en dollars, a de nouveau diminué en pourcentage du PNB pour tomber de $0,34 \%$ en 1978 à $0,30 \%$ en 1979. Le Japon a presque réussi, en 1979, à atteindre l'objectif qu'il s'était fixé pour 1980, à savoir porter le montant en dollars de son APD au double de son niveau de 1977 (1977: 1,4 milliards de dollars; 1979 : 2,6 milliards de dollars). De ce fait, le rapport APD/PNB est passé de $0,23 \%$ en 1978 à $0,26 \%$. Cet accroissement a porté sur tous les éléments du programme d'aide japonais, et plus particulièrement ses dons bilatéraux. Des mesures sont actuellement à l'étude en vue d'accroitre encore I'APD fournie par le Japon. Le rapport APD/PNB notifié pour 1979 par la Finlande, soit 0,21\% contre $0,17 \%$ en 1978 constitue le meil- leur résultat qui ait jamais été enregistré par ce pays; et il y a de bonnes raisons de compter sur un nouvel accroissement de l'effort d'aide finlandais. Pour la Suisse, le rapport correspondant est passé de $0,20 \%$ en 1978 à $0,21 \%$ en 1979 , poursuivant sa tendance régulière, encore qu'assez modeste, à la hausse.

Les statistiques font apparaître une chute brutale du rapport entre I'APD et le PNB des États-Unis, qui est tombé de $0,27 \%$ en 1978 à $0,19 \%$ en 1979 , ce qui est le plus bas niveau qu'il ait jamais enregistré. Toutefois, ces faibles pourcentages donnent une idée exagérée de la diminution effective de l'effort d'aide des ÉtatsUnis. En fait, ceux-ci ont accru leurs versements nets au titre de la plupart des éléments de leur programme d'aide, et le montant de leurs engagements a également été plus élevé qu'en 1978. La dégradation de leurs résultats tient essentiellement à deux causes. D'une part, il y avait eu un rattrapage des émissions de lettres de crédits en faveur d'institutions financières internationales en 1978 (ce qui a gonflé le chiffre des versements des ÉtatsUnis pour cette année-là); d'autre part, les procédures législatives nécessaires à l'émission de nouvelles lettres de crédit n'étaient pas terminées en 1979 (ce qui a fait baisser les chiffres de 1979). Le processus d'émission de lettres de crédit a repris depuis. Ainsi, la somme de $1,1 \mathrm{mil}-$ liard de dollars a été mise à la disposition 


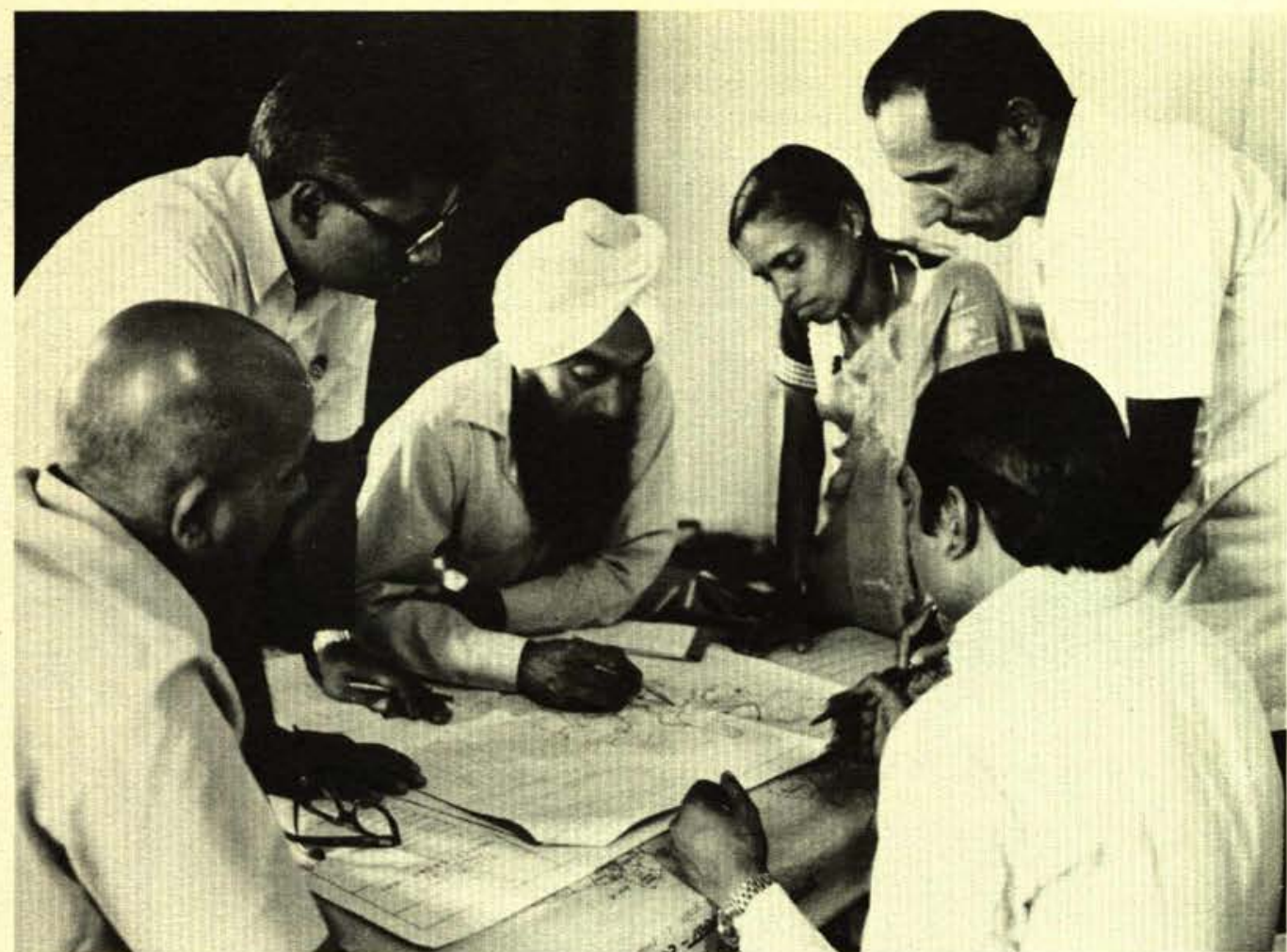

Le peloton de téte: Suède, Pays-Bas, Norvège, Danemark. Ci-dessus : mise sur pied d'un centre sanitaire danois dans le Madhya Pradesh, Inde; Ci-dessous : une clinique en Tanzanie établie grâce à l'aide suédoise.

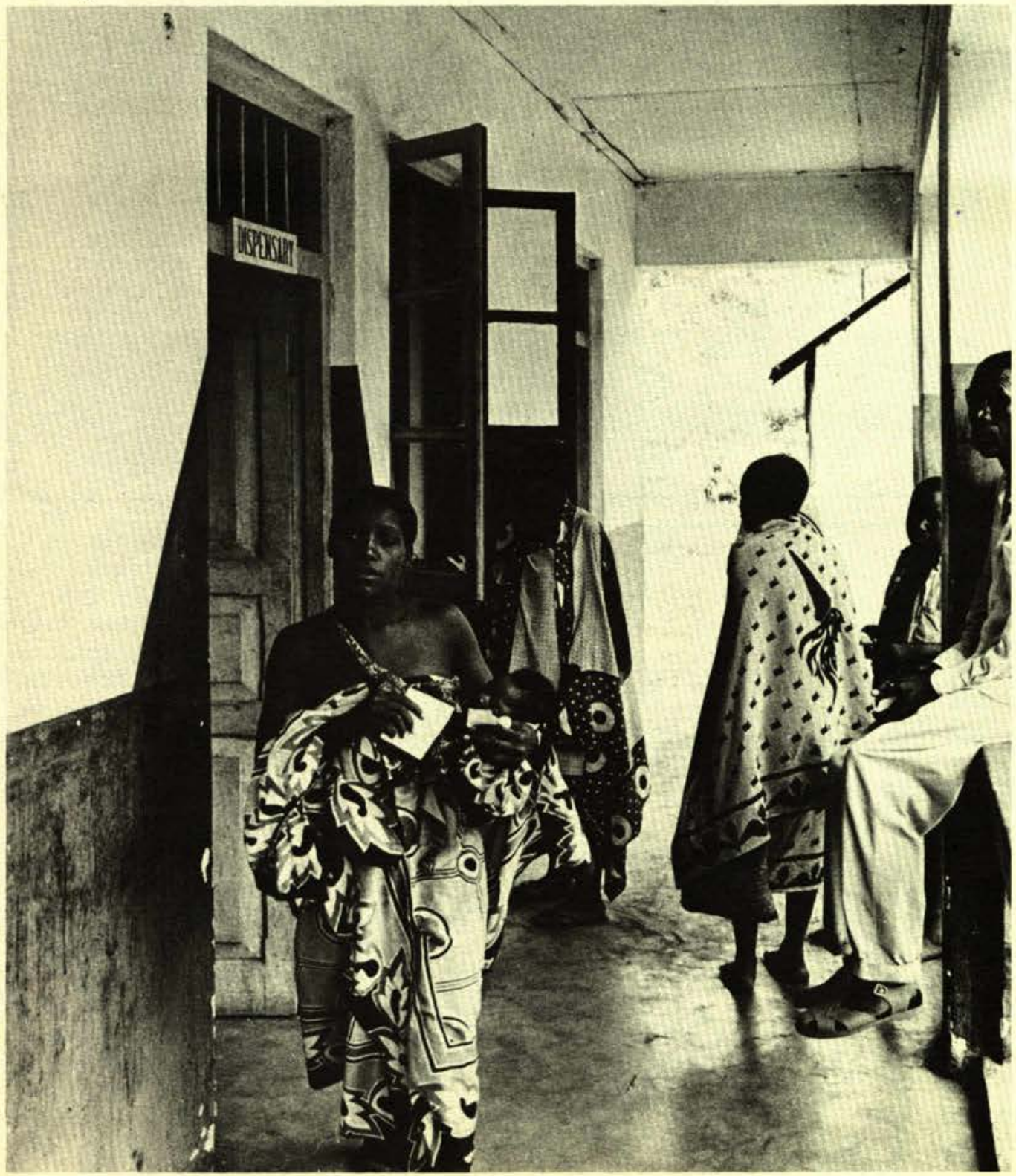

de I'IDA, ce qui suffit à compléter les contributions des États-Unis à la quatrième opération de reconstitution des ressources de I'IDA et à compléter celle de la cinquième reconstitution à raison de $99 \%$. Toutefois, un sérieux problème risque de se poser à propos de la sixième reconstitution des ressources de I'IDA. Pour que celle-ci entre en vigueur, il faudra en effet que les États-Unis aient déposé leurs instruments d'engagement.

Les dons bilatéraux et les contributions multilatérales de l'Autriche ont augmenté, mais les crédits publics à l'exportation assortis de conditions libérales n'ont représenté en 1979 que le quart de leur montant en 1978. En conséquence, l'APD est tombée de 0,29\% du PNB en 1978 à $0,19 \%$ en 1979.

Les versements de I'/talie au titre de l'aide s'élevaient à $0,09 \%$ en 1979 par rapport au PNB. Toutefois, l'administration centrale de l'aide, qui a été créée dernièrement au sein du ministère des Affaires étrangères, a présenté des propositions qui pourraient avoir pour effet de tripler le montant du programme de l'Italie d'ici 3 à 4 ans. Ces propositions ont été bien accueillies au Parlement, qui a voté, pour commencer, une augmentation substantielle des crédits affectés à l'aide pour 1980 , en portant le montant à environ 0,5 milliard de dollars.

\section{L'apport total de ressources des pays du CAD}

D'après les premières estimations, en 1979 l'apport total net des ressources des pays du CAD (aide publique au développement, apports aux conditions du marché des secteurs public et privé et dons du secteur privé) s'est élevé à 70,4 milliards de dollars si l'on exclut les prêts accordés par des banques opérant dans des centres "off-shore" qui sont affiliées à des banques résidant dans les pays du CAD, et à un peu plus de 75 milliards de dollars si I'on tient compte de ces prêts. En dollars, ces deux chiffres sont l'un et l'autre un peu supérieurs aux chiffres correspondants de 1978 (71,4 et 73,4 milliards de dollars), mais ils représentent des pourcentages plus faibles du PNB de l'ensemble des membres du CAD $(1,09 \%$ et $1,16 \%$ contre $1,23 \%$ et $1,31 \%)$.

\section{Donneurs non membres du CAD}

\section{Pays de I'OPEP}

On estime que les versements nets d'aide à des conditions libérales des pays de I'OPEP, dont le montant avait diminué en 1978 d'environ 1,5 milliard de dollars pour tomber à 4,3 milliards, sont remontés à 4,7 milliards en 1979. En pourcentage de leur PNB global, l'aide fournie par les pays donneurs de l'OPEP a enregistré un recul, passant de $1,35 \%$ en 1978 à $1,28 \%$ en 1979 . Ceux de ces pays qui ont 
le revenu le plus élevé (Koweit, Qatar, Arabie Saoudite et Emirats arabes unis) ont pourtant consacré à l'APD une part

\section{APPORTS TOTAUX DE DIFFÉRENTES CATÉGORIES} DE DONNEURS

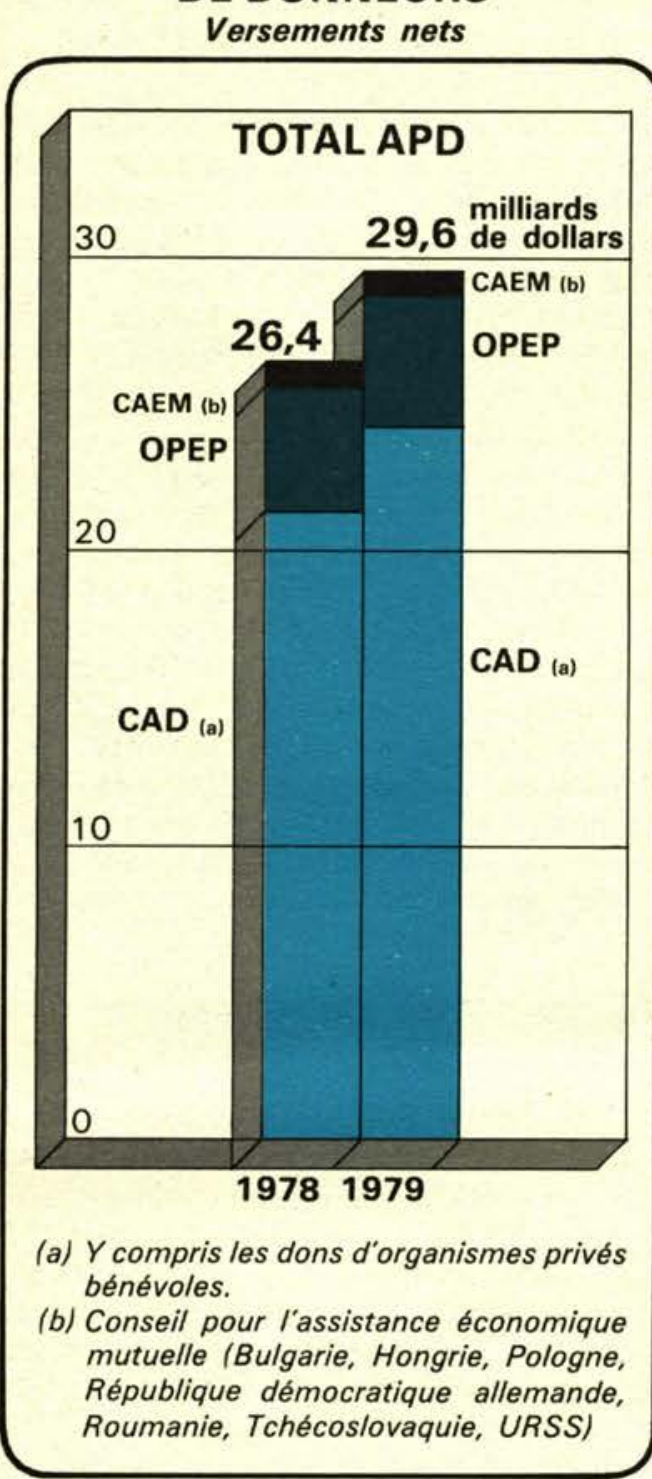

beaucoup plus importante de leur PNB en 1979 (3,48\%) qu'en 1978. Les versements de l'Arabie Saoudite, le plus important des donneurs de I'OPEP, ont augmenté de 0,5 milliard de dollars pour atteindre 2 milliards. Ceux du Koweit sont restés supérieurs à 1 milliard. L'Irak est devenu le troisième donneur de l'OPEP, avec des versements de 0,9 milliard de dollars contre 0,2 milliard en 1978 .

\section{DESTINATION DE L'APD}

a) Par type. 1979. Versements nets : 22,3 milliards de $\$ E U=100 \%$

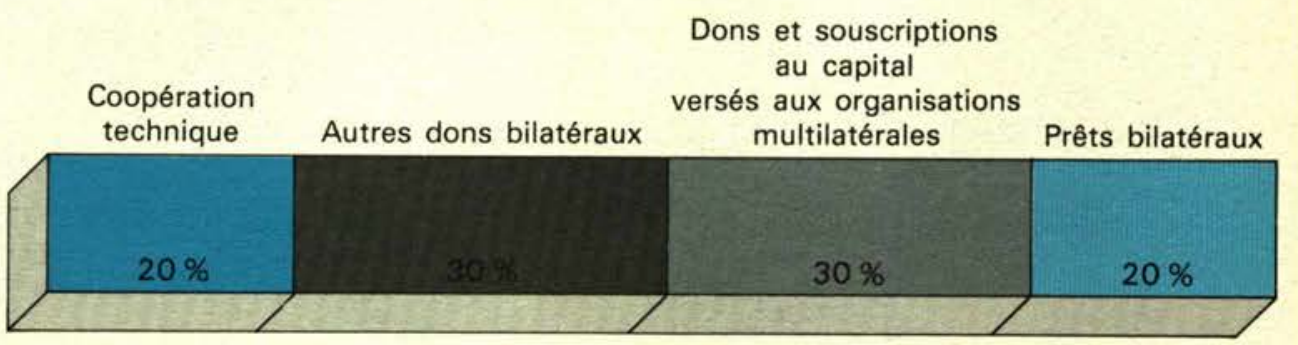

b) Par destination. 1979. Versements nets : 22,3 milliards de $\$ E U=100 \%$

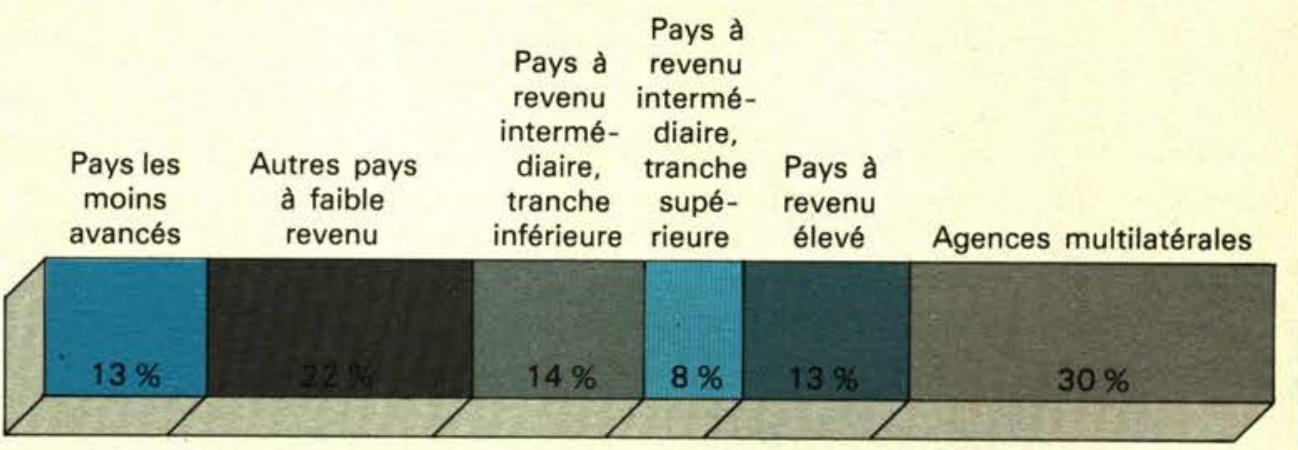

c) Par objet. 1978. Engagements bilatéraux : 18,7 milliards de $\$$ EU $=100 \%$

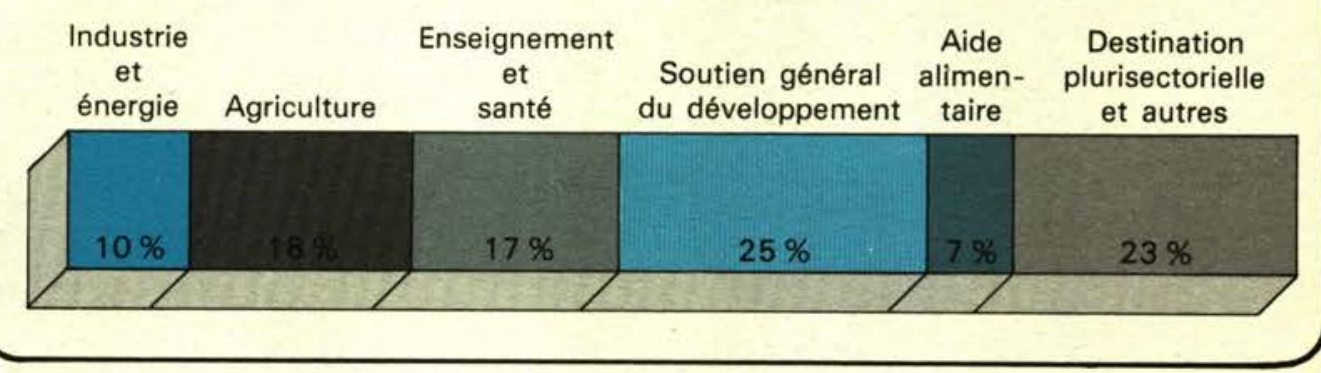

\begin{tabular}{|c|c|c|c|c|c|c|c|}
\hline \multirow{3}{*}{ Pays donneurs } & \multicolumn{4}{|c|}{$\begin{array}{c}\text { APD de l'OPEP (1) } \\
\text { Versements nets }\end{array}$} & & & \\
\hline & \multicolumn{5}{|c|}{ En pourcentage du PNB } & \multicolumn{2}{|c|}{ millions de $\$$} \\
\hline & 1975 & 1976 & 1977 & 1978 & 1979 & 1978 & 1979 \\
\hline Algérie & 0,28 & 0,33 & 0,25 & 0,18 & 0,14 & 44 & 45 \\
\hline Arabie saoudite & 5,40 & 5,73 & 4,32 & 2,76 & 3,15 & 1470 & 1970 \\
\hline Iran & 1,13 & 1,13 & 0.27 & 0,33 & 0,03 & 278 & 21 \\
\hline Iraq & 1,65 & 1,44 & 0,32 & 0,76 & 2,94 & 172 & 861 \\
\hline Kuwait & 8,12 & 4,36 & 10,61 & 6,35 & 5,14 & 1268 & 1099 \\
\hline Libye & 2,31 & 0,63 & 0,65 & 0,93 & 0,58 & 169 & 146 \\
\hline Nigeria & 0,05 & 0,25 & 0,16 & 0,08 & 0,05 & 38 & 28 \\
\hline Qatar & 15,62 & 7,95 & 7,93 & 3,65 & 5,60 & 106 & 251 \\
\hline \multicolumn{8}{|l|}{ Union des } \\
\hline Emirats arabes & 14,12 & 11,02 & 10,22 & 5,60 & 1,58 & 690 & 207 \\
\hline Venezuela & 0,11 & 0,33 & 0,14 & 0,28 & 0,18 & 109 & 83 \\
\hline TOTAL & 2,71 & 2,27 & 1,96 & 1,35 & 1,28 & 4344 & 4711 \\
\hline
\end{tabular}

En ce qui concerne les composantes de l'aide des pays de l'OPEP, leurs dons multilatéraux ont augmenté de 0,5 milliard de dollars pour atteindre 2,7 milliards. Leurs vesements au titre de prêts bilatéraux ont légèrement progressé mais leurs contributions aux institutions multilatérales ont fléchi à 1,2 milliard de dollars.

\section{Pays du CAEM (4)}

Sur la base des informations limitées disponibles, on estime qu'en 1979 les versements nets de I'URSS et des six pays d'Europe de l'Est ont représenté 0,7 milliard de dollars, soit un pourcentage du PNB qui demeure au bas niveau de $0,04 \%$. Ces chiffres ne tiennent pas compte de l'aide substantielle, mais dont le montant est inconnu, accordée à Cuba.

(4) CAEM (Conseil pour l'assistance économique mutuelle): Bulgarie, Tchécoslovaquie, RDA, Hongrie, Pologne, Roumanie et URSS. 


\section{L'apport total de ressources aux pays en développement}

Les apports de ressources analysés plus haut comprennent les apports des pays du CAD, les prêts bancaires accordés par l'intermédiaire de centres financiers "offshore", les apports des pays de l'OPEP et ceux des pays du CAEM. On estime pour le moment qu'en 1979 le montant en dollars de l'apport total de ressources (82 milliards) n'a que très peu augmenté par rapport à 1978 (81 milliards). Les apports des pays du CAD (y compris les prêts des banques établies dans des centres "off-shore" et affiliées à des banques des pays du CAD) ont représenté $92 \%$ du total.

II est intéressant de noter que l'apport total de ressources (à des conditions libérales et aux conditions du marché) exprimé en pourcentage du PNB de l'ensemble des pays en développement est passé d'environ $3 \%$ au début des années 1970 à plus de $5 \%$; à l'intérieur de ce total, l'aide publique au développement fournie au groupe de pays "les moins avancés" exprimée en pourcentage du PNB de ces derniers a progressé régulièrement, de $4 \%$ au début de la décennie à plus de $10 \%$ en 1978 ; ce niveau, d'après les indications partielles actuellement disponibles, a été au moins maintenu en 1979.

\section{La dette des pays en développement}

L'augmentation de la dette nominale de l'ensemble des pays en développement s'est accélérée durant la crise pétrolière de 1973-75, elle est restée rapide au cours de la période 1976-78 durant laquelle elle a également été liée à un important accroissement des réserves extérieures, et elle s'est de nouveau accélérée en 1979.

Au cours de la dernière décennie la part de la dette envers les pays du CAD, y compris la dette née d'emprunts sur les

\section{COOPÉRATION CAD/PAYS ARABES}

Des représentants d'organismes d'aide arabes et des membres du Comité d'aide au développement (CAD) se sont rencontrés au siège de l'OCDE du 18 au 20 juin 1980, pour la troisieme fois à un an d'intervalle.

A cette occasion, huit organismes d'aido arabes étalent représentés, pour la plupart par leur président ou leur directeur exécutif, la participation des membres du CAD étant de niveau équivalent. La Banque mondiale, le FMI et la Banque africaine de développement étaient également représentés. Abdlatif Al Hamad, directeur général du Fonds koweitien pour le développement économique arabe, et John P. Lewis, président du CAD, ont assuré conjointement la prásidence de la réunion. Les débats ont essentiellement porté sur les problèmes opérationnels que rencontrent les donneurs pour ce qui est de leurs programmes d'aide destinés aux pays de l'Afrique sub-saharienne, région dont les problèmes et les besoins prennent, de l'avis général, une acuité croissante.

L'objet de ces réunions est non seulement de fournir l'occasion d'une discussion libre et informelle sur les problèmes de cette nature, qui intéressent l'ensemble des donneurs d'aide, mais aussi de faciliter les contacts personnels entre responsables des organismes d'aide. Un des résultats de la collaboration entre les deux groupes est le nombre croissant des projets financés conjointement. Ces dernières années, plus de 120 projets cofinancés par des membres du CAD et des organismes d'aide arabes

ont vu le jour.

narchés des capitaux internationaux, est restée de l'ordre de $75 \%$ à $80 \%$, mais les créances du secteur privé, qui sont toutes assorties des conditions du marché, en représentent maintenant la moitié au lieu du quart. La part dans le total de l'encours de la dette envers les pays de l'OPEP a augmenté pour atteindre $4 \%$ (mais la part de la dette due par les pays de I'OPEP a doublé pendant cette période pour atteindre $25 \%$ ).

Le montant nominal de la dette est constitué par la somme des crédits non remboursés pour lesquels les paiements de service de la dette présentent des profils très différents : l'important pour les débiteurs comme pour les créanciers c'est que les paiements dus chaque année à ce titre restent dans les limites de la charge supportable par les emprunteurs. Bien que ces paiements aient fortement augmenté en valeur nominale au cours de la décennie 1970, le "coefficient de service de la dette" (rapport en pourcentage entre le service de la dette et le total des recettes d'exportation) n'a pas en moyenne beaucoup varié (encore que cette moyenne de quelque $16 \%$ masque d'importantes différences d'un pays à l'autre). Durant toute la décennie 1970, les paiements au títre du service de la dette née de crédits à l'exportation et de la dette née d'emprunts aux marchés des capitaux ont constitué la majeure partie du total. Ils en représentaient ensemble environ $70 \%$ au début des années soixantedix, mais cette proportion s'élève maintenant à plus de $80 \%$.

En 1979, les deux pays les plus lourdement endettés, le Brésil et le Mexique, comptaient pour $\mathbf{3 0} \%$ dans le total du service de la dette. Les dix pays les plus lourdement endettés, comprenant non moins de cinq pays de l'OPEP (Iran, Algérie, Arabie Saoudite, Indonésie et Venezuela), comptaient pour près des trois-quarts dans le total de la dette des pays en développement.

\section{L'AIDE EN 1979}

commentée par John Lewis, président du Comité d'aide au développement de l'OCDE (CAD)

\begin{abstract}
e bilan de l'aide pour 1979 est préoccupant. Pour deux raisons. Les sommes versées pendant l'année sont déjà loin d'être extraordinaires, mais, en plus, si l'on cherche à voir un peu plus loin - en examinant notamment les chiffres des engagements - on s'aperçoit que, même dans le cas d'une amélioration prochaine, l'aide sera probablement loin de répondre aux besoins. Pour l'ensemble des pays membres, c'est pratiquement le point mort en ce qui concerne l'aide publique au développement (APD) exprimée en pourcentage du PNB - l'élément auquel le CAD s'attache le plus. Le rapport APD/PNB a tout juste glissé d'un peu moins de $0,35 \%$ à un peu plus de $0,34 \%$, c'est-à-dire qu'il n'y a pas eu de changement.
\end{abstract}

Ce résultat est particulièrement peu satisfaisant à un moment où le pourcentage devrait augmenter. L'aide aux pays les plus démunis était déjà insuffisante avant le choc pétrolier. Depuis lors, ces pays ont pris de plus en plus de retard, si bien que leurs besoins de développement pour les années quatre-vingts sont immenses. Maintenant, il faut en plus compter avec le renchérissement du pétrole et ses répercussions indirectes sur les pays importateurs de pétrole. Les déficits des balances des paiements de ces derniers prennent des proportions effrayantes, et les pays les plus démunis n'ont que peu d'accès aux ressources financières aux conditions du marché. Ils sont donc forcément tributaires, pour l'essentiel, de l'APD. II est indispensable que l'aide destinée 


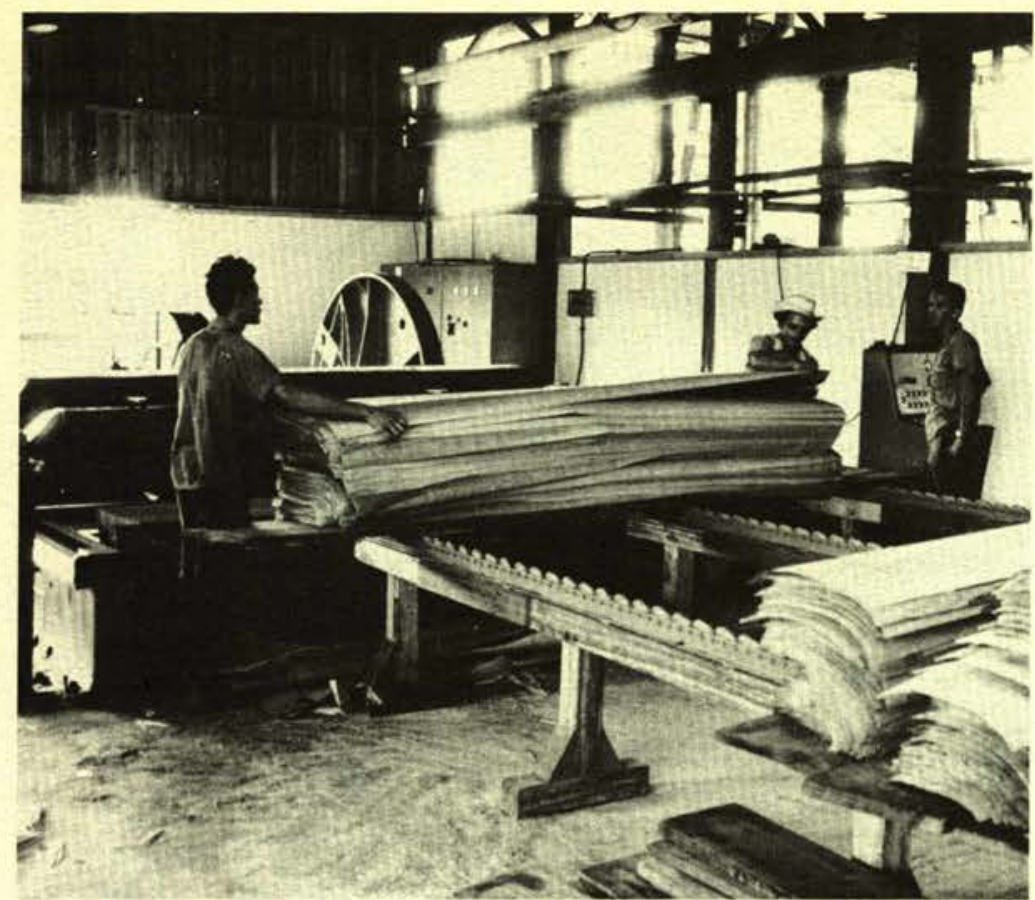

C'est l'aide allemande qui a le plus progressé. Ci-dessus: projet en Colombie destiné à protéger la forêt et créer des emplois locaux.

à ces pays exprimée en pourcentage du PNB, double d'ici à 1985 . II n'existe pas, à ma connaissance, d'étude ayant conclu que l'aide qui leur est accordée est suffisante.

II y a un an, je pensais que l'aide aurait plutôt tendance à monter qu'à baisser et j'espérais que pendant plusieurs années elle pourrait s'accroître de façon appréciable. Pour savoir ce qui s'est en fait passé, il faut considérer la situation des différents pays et groupes de pays. D'abord, le peloton de téte : les quatre pays qui en font partie - la Suede, les Pays-Bas, la Norvège, le Danemark - ont vaillamment continué sur leur lancée. II y a bien quelques changements mais, fondamentalement, ces pays montrent la voie que tous les donneurs devraient suivre. Vient ensuite le groupe intermédiaire : ces pays maintiennent à peu près leurs résultats, avec un léger progrès pour certains, un léger recul pour d'autres. La France et la Belgique, notamment, augmentent quelque peu leurs contributions. Le Royaume-Uni constitue - on le verra plus loin - un cas à part ; son aide publique a, en fait, fortement augmenté de 1978 à 1979. Parmi ceux qui ont cédé un peu de terrain, on trouve l'Australie et la Nouvelle-Zélande. En bas de liste, l'ordre s'est quelque peu modifié. L'Italie accorde désormais une plus grande attention à l'aide et cela commence à apparaître dans les chiffres; cependant, le niveau reste très faible. L'Autriche a marqué un net recul du fait d'une diminution des versements au titre des crédits à l'exportation assortis de conditions libérales. Si, enfin, les résultats de la Finlande sont encore modestes, l'aide de ce pays suit pourtant une pente nettement ascendante. Le rapport APD/PNB - de 0,17 et $0,16 \%$ les deux années précédentes est en effet passé en 1979 à $0,22 \%$, le meilleur niveau jamais atteint. Son programme est bien conçu et bien mené, et l'on espère que c'est le début d'une progression soutenue.

Le pays qui remporte la palme est l'Allemagne : en faisant passer le rapport APD/PNB de 0,33 à $0,44 \%$ en deux ans, elle a réalisé un progrès considérable qui non seulement représente d'ores et déjà une nette amélioration, mais qui indique aussi dans quel sens les autorités entendent à l'avenir orienter leur politique. Pour les trois années qui viennent, l'Allemagne s'est engagée à augmenter le volume de son APD, soit deux fois plus vite que le budget global, soit à une cadence annuelle de $12 \mathrm{r} / 2$; l'on peut en déduire que cette tendance devrait se poursuivre. II s'agit là d'un engagement remarquable. L'Allemagne se fait aussi fort d'atteindre l'objectif de $0,7 \%$ d'ici à la fin de la décennie, ce qui témoigne d'une réorientation très profonde et très intéressante de sa politique.

Dans le cas du Japon, l'évolution est moins spectaculaire, mais les Japonais vont certainement tenir l'engagement qu'ils ont pris, il y a trois ans, de doubler entre 1977 et 1980 le volume de I'APD en dollars courants. II faut noter cependant que le PNB de ce pays s'accroît plus vite qu'ailleurs et que le rapport APD/PNB n'augmentera par conséquent pas dans des proportions extraordinaires ; il est néanmoins passé de $0,21 \%$ il y a deux ans à $0,26 \%$ en 1979 et l'on espère qu'il va continuer de progresser.

Les résultats obtenus par le Royaume-Uni en 1979 pourraient paraître surprenants, si l'on en jugeait d'après les orientations actuelles en la matière. Cela tient au décalage qui se produit entre les engagements et les versements. Ces derniers ont considérablement augmenté, leur part dans le PNB ayant progressé de 0,04 point - de 0,48 à $0,52 \%$ - et, par rapport à il y a deux ans, l'accroissement s'établit à non moins de 0,15 point. Cette augmentation des versements a été observée pour la plupart des composantes du programme d'aide, qu'il s'agisse des contributions bilatérales ou multilatérales, et elle correspond à une série de décisions positives prises il y a deux ou trois ans, dont les effets, synchronisés dans le temps, se sont additionnés. Rien cependant ne permet de dire ce qui va se passer dans la période qui vient. Le Royaume-Uni prévoit pour les prochaines années une réduction sensible de l'APD en termes réels, ce qui veut dire que la part de celle-ci dans le PNB sera encore plus faible.

Quant aux États-Unis et au Canada, ils ont déjà enregistré une diminution en valeur réelle de leurs versements en 1979 et ce sont les résultats de ces deux pays - du premier surtout, parce que le programme américain est toujours de loin le plus important en termes absolus - qui ont le plus pesé sur les résultats globaux des membres du CAD l'année dernière. Le rapport APD/PNB du Canada est beaucoup plus élevé que celui des États-Unis. II peut se comparer à celui du groupe intermédiaire, à savoir la France, la Belgique, le Royaume-Uni, I'Australie et l'Allemagne. Le recul absolu observé l'année dernière traduit la réaction assez rapide des versements à la décision prise, à la fin de l'été, de ralentir les engagements. Actuellement, les perspectives d'évolution à moyen terme de l'aide canadienne sont incertaines, mais le ferme attachement que ce pays a toujours manifesté à la cause du développement permet d'espérer.

Dans le cas des États-Unis, la chute brutale du rapport APD/PNB - tombé de $0,27 \%$ en 1978 à $0,19 \%$ seulement en 1979 - exagère les données réelles de la situation. II y a d'abord une question de comptabilisation, un changement de méthode décidé d'un commun accord au sein du CAD ayant eu pour effet de rendre la comparaison entre 1978 et 1979 un peu plus défavorable qu'elle ne l'est en réalité (1). Mais il faut surtout tenir compte du fait que les transferts des États-Unis à la Banque mondiale et d'autres institutions multilatérales ont été concentrés sur 1978 , alors que diverses difficultés et complications d'ordre législatif n'ont pas permis au gouvernement de disposer des pouvoirs nécessaires pour procéder à des opérations analogues en 1979 . Celles-ci ont aujourd'hui repris, mais les problèmes posés dans ce pays par les contributions multilatérales demeurent préoccupantes pour l'avenir, et, si l'on en juge par les prévisions budgétaires actuelles, le rapport engagements/PNB devrait en moyenne être plus faible pendant les quelques prochaines années.

Cette dernière évolution, dont les effets se conjuguent au fléchissement attendu pour le Royaume-Uni et à la hausse prévue dans le cas de l'Australie, de la Nouvelle-Zélande et peut-être du Canada, va peser sur les résultats globaux des membres du CAD au cours des années qui viennent. II n'est pas certain que l'expansion des programmes d'aide de l'Allemagne, du Japon et de quelques autres pays arrivera à contrebalancer ces incidences négatives. Même si c'était le cas, on ne peut guère s'attendre que les résultats moyens du CAD progressent au cours des prochaines années à un rythme qui se rapproche de celui qui serait nécessaire.

(1) En 1979, les pays du CAD ont normalisé la comptabilisation des contributions versées aux organismes multilatéraux, de manière que celles-ci soient enregistrées par tous - et non plus seulement par quelques-uns - à la date du dépót, et non plus de l'encaissement, des effets représentatifs de ces contributions. 


\section{VERS UNE REGLEMENTATION INTERNATIONALE DES PRODUITS CHIMIQUES TOXIQUES}

es produits chimiques présentent l'un des grands paradoxes du vingtième siècle. Au cours des trente dernières années, les progrès des sciences chimiques ont probablement apporté une plus grande contribution à l'amélioration de la qualité de la vie dans la société moderne que toute autre technologie. II est peu d'industries auxquelles les produits chimiques ne soient pas utiles et les progrès en matière de médicaments, de produits agricoles et de produits de consommation sont légion. Cependant ces progrès ne se traduisent pas seulement par la mise au point de produits pharmaceutiques qui sauvent des vies humaines et de produits qui rendent la vie plus facile, mais aussi par une pollution de l'environnement et par une perception de plus en plus nette des effets négatifs à long terme qu'a sur la santé humaine l'accumulation de produits chimiques. Dans tous les pays de I'OCDE, les pouvoirs publics sont de plus en plus conscients des dangers potentiels de ces produits pour l'homme et pour l'environnement. Mais on est également conscient, surtout dans le climat économique actuel, de l'importance du rôle économique de l'industrie chimique -300 milliards de dollars de chiffre d'affaires annuel, 80 milliards d'exportations, 4 millions d'emplois - et donc de la nécessité de concilier les préoccupations relatives à l'environnement et celles liées à la croissance économique.

Pour protéger l'homme et l'environnement, les gouvernements des pays de I'OCDE prennent des mesures nouvelles et plus strictes en vue non seulement de maîtriser les émissions et les rejets de déchets, comme c'était le cas auparavant, mais aussi pour empêcher que des substances dangereuses soient introduites sur le marché et pour réglementer leur utilisation. En outre, plutôt que de porter leur attention sur les seuls produits chimiques connus pour être dangereux, comme les PCB et le mercure, ou sur des groupes déterminés de substances, comme les pesticides, les produits pharmaceutiques ou les additifs alimentaires, les autorités s'intéressent aujourd'hui à toute la gamme des produits chimiques. L'éventail des dangers envisagés s'est également élargi pour inclure non seulement la toxicité aiguë mais aussi les effets à long terme comme l'apparition de cancers (pouvoir cancérigène), ainsi que des phénomènes de plus en plus complexes tels que la persistance dans l'eau et dans l'air, l'accumulation dans la chaîne alimentaire et les dommages possibles aux conditions mêmes qui entretiennent la vie sur la terre.

\section{L'histoire d'une législation}

La législation concernant les produits chimiques devient de plus en plus fréquente. La "doyenne» des lois a été adoptée en Suisse en 1969; elle consiste à classer chaque produit chimique introduit sur le marché suisse selon son danger potentiel pour la santé humaine en termes de toxicité aiguë, toutefois sans tenir compte de ses effets sur l'environnement. En 1973, le Japon, qui avait récemment subi la catastrophe de la baie de Minimata, due au mercure, et la Suède, qui a toujours été à l'avant-garde en matière d'environnement, ont également adopté des lois visant à étendre la réglementation des produits chimiques et prenant en compte des considérations d'environnement. Le Royaume-U̇ni, le Canada et la Norvège ont pris la même voie en 1974 et 1975 , et en 1976 l'adoption par les États-Unis - le plus important producteur de produits chimiques du monde et l'un des principaux importateurs et exportateurs - de la loi sur le contrôle des substances toxiques, a donné une vive impulsion aux mesures législatives en ce domaine. Début 1978, la France a adopté une législation analogue. D'autres pays, ainsi que les Communautés européennes, s'apprêtaient à faire de même. Les inconvénients d'une action des pays en ordre dispersé devenaient en même temps plus qu'évidents. Et cela pour plusieurs raisons.

\section{Les inconvénients d'actions séparées}

Tout d'abord, le nombre de produits chimiques à réglementer est véritablement énorme. Personne ne sait exactement combien de dizaines de milliers sont déjà sur le marché, et plus d'un millier de produits nouveaux apparaissent chaque année. La réglementation implique des essais, généralement réalisés par l'entreprise productrice, et une évaluation, à laquelle procèdent l'industrie ou les pouvoirs publics. Les essais exigent des ressources importantes, non seulement en termes monétaires (le coût des essais va de quelques milliers à quelques centaines de milliers de dollars, mais aussi en termes de laboratoires et de personnel scientifique, actuellement peu abondant (il y a par exemple une pénurie mondiale de toxicologues). Si chaque pays exigeait la réalisation d'essais différents - comme cela semblait probable à un certain moment - les coûts seraient beaucoup plus élevés pour une entreprise qui souhaite commercialiser ses produits à l'étranger. Pour certaines petites entreprises, peut-être novatrices, les coûts des essais pourraient être prohibitifs. Les coûts administratifs, visant à faire en sorte que les essais et l'évaluation soient corrects, sont également élevés. Pour les gouvernements, notamment des petits pays, la collecte des renseignements et la surveillance des produits chimiques pourraient poser des problèmes considérables. Même si une législation stricte était en vigueur, la mise en application de celle-ci serait difficile du point de vue pratique en raison du personnel et du temps nécessaires pour l'examen des dossiers relatifs aux produits chimiques.

II y avait tout lieu de craindre que les nouvelles réglementations, si elles n'étaient pas coordonnées, puissent bouleverser les échanges de produits chimiques et entraîner l'apparition d'obstacles non tarifaires et d'autres formes de protectionnisme. Par exemple, lorsque le Japon a exigé que l'on vérifie qu'un produit est biodégradable, qu'il ne s'accumule pas dans la chaîne alimentaire et qu'il n'est pas toxique, certains fabricants ne savaient initialement pas comment réaliser de tels essais, et il leur a fallu un certain temps pour être à même de répondre aux réglementations japonaises et par conséquent d'exporter à ce pays.

\section{La poussée vers la coopération}

Ayant à l'esprit ce type de considérations, des spécialistes de l'environnement et des hauts fonctionnaires de seize pays et de six organisations internationales se sont réunis à Stockholm en avril 1978, pour examiner ce qui pouvait être fait pour promouvoir cette forme de protection de l'environnement, d'une manière qui ne perturberait pas les échanges et n'imposerait pas des coûts intolérables à l'industrie chimique. Les participants à cette réunion ont confié à l'OCDE la mission de renforcer, pour une période de trois ans, un programme déjà existant relatif aux produits chimiques.

Le programme de I'OCDE, commencé en 1970, portait initialement sur des substances déterminées, comme les PCB et le mer- 
cure (en 1973, le Conseil de I'OCDE a décidé qu'il y avait lieu d'interdire la plupart des utilisations des $\mathrm{PCB}$ et a recommandé une réduction des émissions de mercure), mais son champ d'action avait déjà été élargi en réponse aux préoccupations croissantes que manifestaient les pouvoirs publics au sujet de l'ensemble de la gamme des produits chimiques. En 1974, le Conseil a recommandé une évaluation et une réglementation étendues des produits chimiques ainsi que l'harmonisation des systèmes adoptés par les différents pays. Le Comité de l'environnement de I'OCDE travaillait déjà dans cette direction, mais avec des ressources limitées.

En octobre 1978, le programme élargi, axé sur les produits chimiques nouveaux, a été lancé avec l'aide de fonds, d'experts, de laboratoires etc. fournis par les dix-neuf gouvernements qui ont choisi de participer au programme spécial, ainsi que par la Commission des Communautés européennes. L'un des objectifs principaux est d'élaborer un système international d'essai et d'évaluation qui permettra à un gouvernement d'accepter les données d'essai d'un produit chimique nouveau obtenues dans un autre pays. Du point de vue des exportateurs, cela signifie que les résultats d'une série unique d'essais d'un produit nouveau seraient valables pour l'évaluation dans tous les autres pays de I'OCDE, tandis que les importateurs pourraient se fier aux données sur un produit nouveau reçues de l'exportateur.

\section{L'élaboration d'un ensemble d'actions}

La première étape consistait à élaborer un manuel sur la façon de tester les produits chimiques, ce que le professeur SchmidtBleek, directeur de l'Agence allemande de l'environnement, responsable d'une partie du projet, a appelé «le livre de cuisine». En 1000 pages, le manuel, sous sa forme actuelle, décrit comment, concrètement, les laboratoires devraient procéder aux essais relatifs à quelque 100 propriétés, dont la biodégrabilité et l'accumulation dans l'environnement, le pouvoir cancérigène, la toxicité et les effets sur la reproduction. Ces descriptions devraient permettre à tout laboratoire disposant d'un personnel et d'équipements appropriés de réaliser les essais demandés par les pouvoir publics.

\section{QUELQUES LÉGISLATIONS SUR L'ESSAI DES PRODUITS CHIMIOUES NOUVEAUX}

\section{Communautés européennes}

Conformément à la sixième modification de la directive sur la classification, l'emballage et l'étiquetage des substances dangereuses, une entreprise chimique sera tenue d'avertir les autorités nationales compétentes quarante-cinq jours avant qu'elle se propose d'introduire un produit chimique nouveau sur le marché. (Un produit chimique nouveau est défini comme un produit commercialisé après septembre 1981, date qui est également l'échéance pour laquelle les pays de la CEE devront avoir donné force de loi à la directive.) La notification doit comporter un «ensemble minimal" ou "dossier de base " de données ou de résultats d'essais (appelé niveau 0). Au cours de la période de quarante-cinq jours, les autorités compétentes doivent examiner le dossier en vue de s'assurer de sa conformité aux prescriptions de la directive et notamment vérifier les conclusions du notifiant quant aux risques prévus, à la classification, à l'étiquetage et aux précautions recommandées de sécurité. Si aucune lacune ou erreur n'est relevée dans le dossier avant l'échéance de quarante-cinq jours, l'entreprise peut introduire le produit sur le marché. Si des défauts sont constatés, le dossier doit d'abord être corrigé. Les données communiquées au gouvernement d'un pays membre du Marché commun seront accessibles aux autres gouvernements pour examen. Si l'un de ceux-ci constate un défaut, il en avisera le premier gouvernement, qui en informera alors l'entreprise. Si l'organisme de contrôle découvre quelque raison de penser que le produit chimique présente un danger, il peut prendre des mesures appropriées relatives à la sûreté d'utilisation du produit, dans
I'attente de l'application des dispositions communautaires. L'hypothèse est donc que l'entreprise prendra grand soin de réaliser les essais de façon rigoureuse.

Lorsque la production atteint 100 tonnes par an, l'entreprise doit soumettre un deuxième ensemble de résultats d'essais (appelé niveau 1), et lorsqu'elle atteint 1000 tonnes par an, un troisième "panier» (niveau 2), plus orienté vers les éventuels dommages de grande ampleur pour l'environnement.

\section{États-Unis}

Conformément à la section cinq de la loi sur le contrôle des substances toxiques, adoptée en 1976, les mesures de contrôle sont mises en œuvre avant la fabrication et non avant la commercialisation comme au sein du Marché commun. Une entreprise qui désire produire un nouveau composé chimique doit informer I'EPA quatrevingt-dix jours avant le début de la fabrication.

Le fabricant est simplement tenu de communiquer ce qu'il sait sur le produit. L'EPA examine alors le dossier en vue de déterminer s'il existe ou non quelque indication que le produit présente un danger potentiel. Si elle estime que les renseignements sont insuffisants, elle peut demander des informations complémentaires, mais l'entreprise peut repousser cette demande et porter la question devant les tribunaux, comme cela est effectivement arrivé dans un cas récent. L'EPA soutenait que les composés en question - une série d'esters de phtalate - étaient chimiquement analogues à certains composés dont on avait montré qu'ils pouvaient être cancérigènes et l'entreprise a retiré la notifi- cation plutôt que de se conformer aux prescriptions formelles. Si I'EPA n'a mis en évidence aucun danger potentiel à l'échéance des quatre-vingt-dix jours, l'entreprise peut entamer la production du composé chimique, celui-ci est ajouté à l'inventaire des produits chimiques existants et peut-être fabriqué librement. Si des dangers sont découverts, I'EPA a le pouvoir de réglementer l'utilisation ou d'interdire à l'entreprise de produire le composé chimique nouveau.

\section{Japon}

Une législation existe depuis 1974 en vertu de laquelle une entreprise chimique, qui désire fabriquer ou exporter un produit, doit informer le ministère de la Santé et le ministère du Commerce et de l'Industrie de son intention et des résultats d'au moins un parmi trois ensembles d'essais : biodégradabilité, bio-accumulation et toxicité. Le premier d'entre eux est de loin le moins coûteux et il est par conséquent généralement choisi. Si les autorités responsables de la réglementation constatent que le produit est biodégradable, celui-ci fera l'objet d'une publication au journal officiel et l'entreprise pourra commencer la fabrication. Si l'on constate le contraire, la firme doit procéder à un deuxième essai, qui porte généralement sur la bioaccumulation; celui-ci est plus côteux que l'essai de biodégradabilité, mais moins que celui de toxicité. Les produits chimiques qui sont définis selon le processus comme persistants, susceptibles de bio-accumulation et toxiques font l'objet d'une publication au journal officiel en tant que produits chimiques "classés" et leur importation ou leur fabrication est en principe interdite. 
Pour que les résultats soient acceptés - par les autorités nationales responsables de la réglementation ou par d'autres gouvernements - les pouvoirs publics doivent avoir l'assurance que le laboratoire travaille effectivement en conformité avec les normes de qualité convenues et que les essais sont réalisés de façon correcte. C'est pourquoi, en plus de l'élaboration de lignes directrices pour les essais, les travaux de l'OCDE ont porté simultanément sur la mise au point d'un code de bonnes pratiques de laboratoire qui est à présent achevé.

Troisième partie de ce travail initial : décider lesquels parmi les 100 essais étaient susceptibles de fournir les données dont l'industrie et les pouvoirs publics ont besoin pour procéder à une première évaluation significative avant l'introduction sur le marché d'un produit chimique. Cet «ensemble minimal de données préalables à la commercialisation" permettra de déterminer si un produit chimique nouveau est ou non susceptible de présenter un danger pour l'homme et pour l'environnement.

\section{L'accord des ministres}

A la mi-mai de cette année, alors que la moitié du programme de travail était réalisée, l'ensemble de résultats a été présenté à une réunion à haut niveau du Groupe des produits chimiques de l'OCDE, à laquelle ont participé des ministres de l'environnement et des hauts fonctionnaires responsables des politiques de l'environnement au sens large, ainsi que des personnes spécifiquement chargées des questions touchant aux produits chimiques. A l'unanimité, les participants à cette réunion, que présidait Blair Seaborn, sous-ministre de l'Environnement du Canada, sont convenus d'accélérer les efforts visant à assurer l'acceptation des données entre les pays et ont approuvé l'ensemble des résultats obtenus par l'OCDE, c'est-à-dire les lignes directrices pour les essais, les principes relatifs aux bonnes pratiques de laboratoire et l'ensemble minimal de données préalables à la commercialisation. Selon Rune Lönngren, vice-président de l'Office suédois de la réglementation de produits, qui assistait à la réunion, ce sont là "les outils qui rendront la législation réellement efficace».

Les participants sont également convenus d'appliquer autant que possible ces mesures à l'échelon national avant même qu'elles soient formellement soumises au Conseil de I'OCDE pour adoption en 1981.

Les résultats de la réunion à haut niveau témoignent du réel besoin d'harmonisation que ressentent les pays Membres de I'OCDE au moment où bon nombre d'entre eux formulent de nouvelles lois sur les produits chimiques, où d'autres envisagent de réviser la législation en vigueur et où d'autres encore mettent en application des réglementations d'une portée plus large (1). Les pays du Marché commun doivent par exemple se conformer pour le mois de septembre 1981 à ce que l'on appelle généralement la "sixième modification» de 1979 qui impose la notification et l'essai des produits chimiques nouveaux (voir encadré); les méthodes et les lígnes directrices pour les essais de l'OCDE formeront la base d'une annexe à cette modification relative aux prescriptions en matière d'essai. Les pays qui ont le projet d'adopter de nouvelles lois ou de mettre a jour des lois anciennes se proposent de les formuler conformément au mécanisme d'acceptation mutuelle de I'OCDE. Les États-Unis, où la loi ne définit pas d'exigences formelles pour l'essai des produits chimiques nouveaux, envisagent de publier le texte de I'OCDE sur les prescriptions minimales en matière d'essai ainsi que les descriptions des essais et le manuel des bonnes pratiques de laboratoire en tant que lignes directrices pour les entreprises chimiques. "L'industrie souhaite des indications à cet égard", explique Douglas Costle de l'Agence pour la protection de l'environnement (EPA) des États-

(1) Les pays suivants ont déjà une législation:Suisse (1969), Japon (1973), Suede (1973), Royaume-Uni (1974), Canada (1975), Norvège (1975), États-Unis (1976), France (1977), Danemark (1979) et Nouvelle-Zélande (1979). La CEE a récemment (1979) publié une directive concernant l'essai, la notification et l'évaluation des produits chimiques.
Unis. "Les entreprises se rendent compte qu'au lieu d'avoir à se conformer à cinquante ensembles de prescriptions touchant les échanges internationaux", il leur sera demandé dans chaque pays la même série de données.

\section{Les problèmes non résolus}

Quelques problèmes difficiles doivent encore être résolus pour que l'acceptation mutuelle des données d'essais devienne une réalité. L'un d'entre eux touche à la manière d'assurer le respect des normes acceptées de bonnes pratiques de laboratoire. Un autre a trait à l'élaboration d'un mécanisme visant à définir les circonstances et les conditions dans lesquelles des essais complémentaires sont nécessaires après l'évaluation initiale, par exemple selon une méthode séquentielle ou par étapes. Cela permettrait de passer, lorsqu'il y a lieu, de l'ensemble minimal de données préalables à la commercialisation à des essais plus approfondis.

Un troisième problème touche à la façon de tenir les essais à jour. La science de l'évaluation des produits chimiques est relativement neuve et les essais évoluent rapidement, à mesure que l'on met en évidence de nouveaux dangers et que progressent les connaissances relatives à l'incidence des produits chimiques sur I'homme et sur l'environnement. Par conséquent il faut trouver les ressources permettant de faire en sorte que les «lignes directrices» demeurent pertinentes. "Nous nous trouvons au premier échelon d'une haute échelle de connaissancesn, a fait observer Douglas Costle. "Nous n'avons encore qu'une connaissance insuffisante des phénomènes scientifiques que nous étudierons», a ajouté le président de la réunion, Blair Seaborn.

\section{Le caractère confidentiel}

Cependant le plus difficile de tous ces problèmes est peut-être celui qui a trait au caractère confidentiel des données. Le problème réside dans le fait que certaines des données communiquées par l'industrie chimique aux pouvoirs publics touchent à des questions sensibles sur le plan commercial et une bonne part d'entre elles représente des investissements considérables de la part de l'entreprise, en termes de colt des essais et de délais dans la fabrication du produit. Certaines des données peuvent constituer un secret commercial - par exemple le volume de la production - et l'industrie se considère en général propriétaire de l'ensemble des données d'essais. Les travaux d'un groupe d'experts de I'OCDE ont montré que la façon dont ces données sont protégées et traitées diffère entre les pays. Cette situation suscite des problèmes si un gouvernement désire utiliser des données d'essais communiquées à un autre.

Le mécanisme adopté par le Marché commun prévoit l'échange de certaines données entre les Neuf. Lorsqu'une entreprise informe un gouvernement de son intention de commercialiser un produit, le dossier, ou un résumé de celui-ci, sera automatiquement transmis aux huit autres pays. Cependant, même au sein de la CEE, on prévoit certaines difficultés. Pour la zone OCDE beaucoup plus vaste - les problèmes sont plus complexes, et les positions officielles diffèrent.

Certains gouvernements insistent traditionnellement plus que d'autres sur le rôle qu'ils ont à jouer dans la protection des renseignements couverts par la propriété industrielle, des secrets commerciaux ou de la propriété intellectuelle. Les différences entre les politiques des pays en ce qui concerne la divulgation des renseignements administratifs et le droit pour le public de connaître les justifications des décisions des pouvoirs publics qui ont une influence sur la santé et sur l'environnement compliquent encore le problème. On peut dès lors considérer que certains gouvernements sont moins à même que d'autres de protéger contre une divulgation publique les informations couvertes par la propriété industrielle. En théorie, un exportateur pourrait tout simplement renoncer à vendre ses produits dans un pays où il estime que des données dont il est propriétaire ou d'autres renseignements confidentiels risqueraient d'être portés à la connaissance de ses concurrents. La démarche de l'OCDE est autre et tente de conci- 


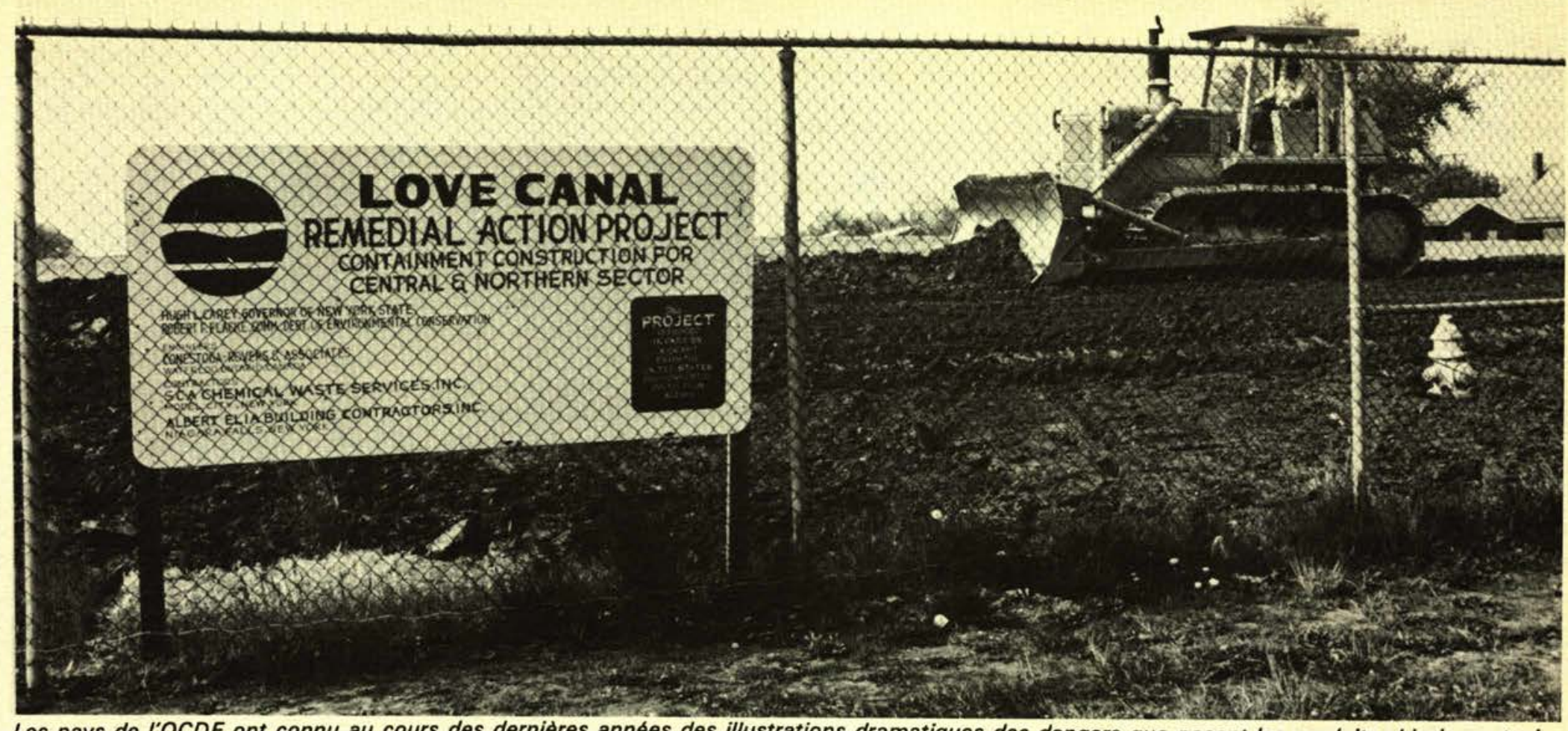

Les pays de l'OCDE ont connu au cours des dernières années des illustrations dramatiques des dangers que posent les produits chimiques toxiques - maladies et déformations foetales provoquées par des déchets immergés dans le Love Canal, New York State (ci-dessus) et par l'échappement de dioxine à Seveso (en bas à gauche). Les sciences chimiques ont cependant fait plus pour améliorer la qualité de la vie que n'importe quelle autre technologie. En bas à droite : la fabrication de produits pharmaceutiques et d'autres produits chimiques en Australie.

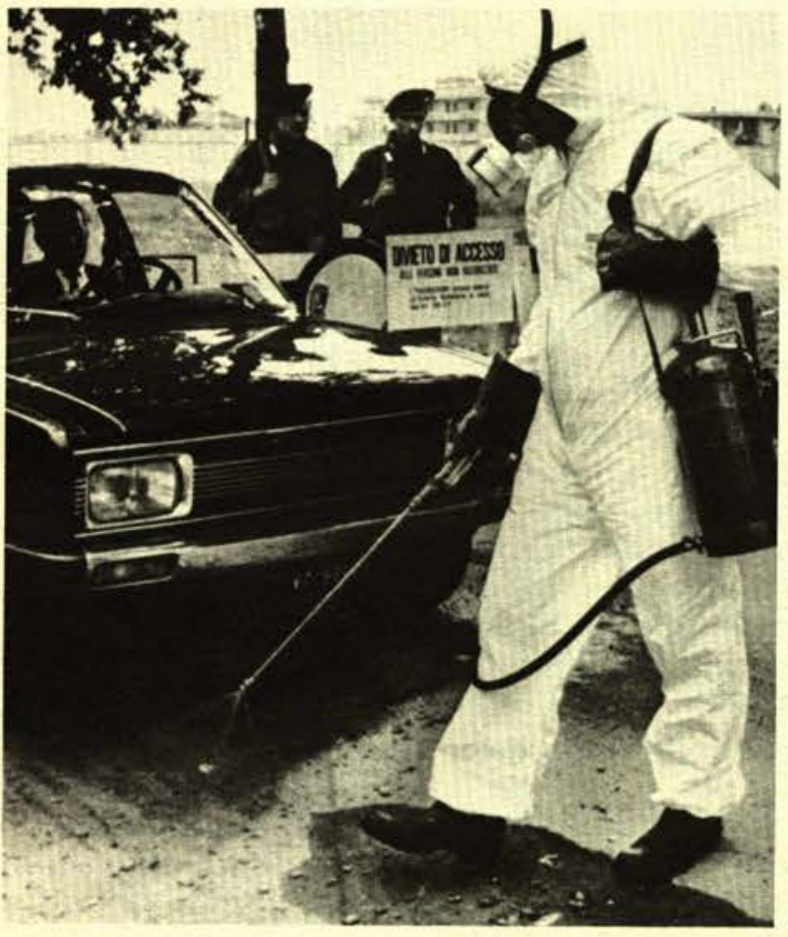

lier des intérêts opposés, afin de permettre la circulation de l'information lorsqu'il y a lieu. C'est pourquoi on étudie le principe d'un accord international qui permettrait aux gouvernements de diffuser les données tout en assurant le secret des renseignements "sensibles» et en protégeant les droits de propriété.

Bien que l'accord n'ait pu se faire sur cette question complexe, la réunion à haut niveau a mis en évidence un terrain d'entente. Comme le disait, R. Robinson, sous-ministre adjoint de l'Environnement, Canada, "nous devons restreindre la définition du caractère confidentiel et élaborer un mécanisme efficace de protection des données réellement confidentielles». Peter Crawford, chef de la Division. des produits chimiques de I'OCDE, a ajouté : «il faut créer un climat tel que, lorsque c'est nécessaire, les données puissent circuler».

Le problème du caractère confidentiel dépasse le cadre de l'acceptation mutuelle des données et les participants à la réunion à haut niveau ont également examiné ses répercussions d'ordre plus général (un groupe d'experts traite de l'échange international d'informations). Le délégué du Canada a souligné "qu'il ne devrait pas y avoir d'obstacles aux échanges de connaissances scientifiques. Nous devons disposer de mécanismes qui fassent en sorte que nous puissions demander des renseignements à d'autres pays et vice-versa, même au sujet de produits qui sont couverts par la propriété industrielle. Nous devons mettre nos connaissances en commun de façon à faire progresser la science de l'évaluation et à réduire les domaines de préoccupation. Douglas Costle, (EPA), lui a fait écho: «Nous avons besoin de techniques assurant la protection du caractère confidentiel, mais nous devons informer le public sur la nature des dangers auxquels il est exposé. Lorsqu'un pays a identifié un danger possible, il est dans I'obligation d'en informer les autres."

\section{Produits chimiques existants}

On a enfin examiné la question de savoir si l'OCDE devrait à l'avenir aborder les problèmes que pose le contrôle des produits 
chimiques existants. Les législations diffèrent à cet égard. Aux États-Unis et au Japon, ces produits sont pris en compte dans les mécanismes de réglementation, mais ils ne le sont pas dans la sixième modification de la directive des Communautés européennes. II ne faut pas sous-estimer les difficultés d'une telle réglementation. En raison du grand nombre de produits chimiques sur le marché (plus de $\mathbf{5 0} \mathbf{0 0 0}$ ont une utilisation commerciale), il est extrêmement difficile de décider quels sont ceux qui doivent être soumis à des essais. Par ailleurs, des pays qui n'ont pas adopté de tels programmes ont estimé nécessaire d'interdire certaines substances bien connues qui précédemment étaient considérées comme "sûres".
Afin que l'on puisse poursuivre l'examen de cette importante question, Günther Hartkopf, secrétaire d'État à l'Intérieur, chef de la délégation allemande à la réunion à haut niveau de l'OCDE, a annoncé que son pays organiserait en avril 1981 à Berlin un symposium sur le contrôle des produits chimiques existants.

"L'important, c'est que la réunion a ouvert la voie à un accord international sur la réglementation des produits chimiques, mais beaucoup reste à fairen, a déclaré Jim MacNeill, Directeur de l'environnement de l'OCDE. "Tous les pays ont réaffirmé leur ferme engagement de poursuivre le programme et ont fourni des orientations précises pour nos travaux futurs. C'était très encourageant", a-t-il ajouté.

\section{REDUIRE LE BRUIT DES VEHICULES A MOTEUR Une conclusion essentielle de la Conférence de l'OCDE}

$\mathbf{U}$ ne conférence de trois jours, organisée récemment par 'OCDE sur le problème du bruit(1), a conclu notamment qu'il y avait lieu de réduire de 5 à 10 décibels d'ici à 1985-90 les limites d'émission sonore applicables aux automobiles, aux poids lourds, aux véhicules de transport en commun, et aux motocyclettes (2).

Pour obtenir une réduction significative de l'exposition au bruit de la circulation, la réduction du bruit à la source est indispensable. Aux États-Unis, par exemple, des prévisions montrent que si rien de plus n'est fait pour réduire le bruit des véhicules à moteur, le nombre de personnes exposées à des niveaux de bruit "inacceptables" (plus de 65 dBA sur l'échelle Ldn)(3); passera de 17,8 millions en 1977 à 21,6 millions en I'an 2000. Si les limites de bruit des poids lourds étaient réduites de $10 \mathrm{dBA}$, et celles des véhicules légers de $5 \mathrm{dBA}$, 6,5 millions de personnes seulement seraient exposées à plus de $65 \mathrm{dBA}$ ( $\mathrm{Ldn}$ ). Certaines estimations européennes montrent de même qu'une réduction généralisée à la source d'environ 10 décibels pour les véhicules lourds et de 5 décibels pour les véhicules légers ramènerait, de $15 \%$ à l'heure actuelle, à 3-5\% environ la proportion des personnes exposées à des niveaux de bruit supérieurs à $65 \mathrm{dBA}$ (Leq).

En Europe et au Japon, les limites d'émission de bruit sont actuellement fixées à $80-81$ dBA pour les automobiles, à 86 88 dBA pour les poids lourds, à 82-91 dBA pour les véhicules de transport en commun et à 81-86 dBA pour les motocyclettes de forte cylindrée(4).

Les gros camions et les véhicules de transport en commun constituent une source majeure de la détérioration de l'environnement sonore et doivent faire l'objet d'une action prioritaire et soutenue. Les motocycles et cyclomoteurs sont une source de gêne d'autant plus importante que le parc continue de s'accroître dans certains pays. Une réduction des limites d'émission de bruit de ces véhicules est également nécessaire. Les émissions sonores des véhicules à deux roues étant largement tributaires des modalités de conduite et de leur entretien, une action complémentaire sur les comportements et l'entretien reste essentielle.

Les limites actuellement appliquées aux nouveaux modèles de véhicules sont insuffisantes pour assurer une protection adéquate de la santé et du bien-être publics. La technologie permet actuellement d'obtenir à un cout raisonnable d'importantes réductions pour les 5 à 10 prochaines années. Les pays Membres devraient donc imposer à ces véhicules les limites les plus strictes possibles sur la base de la meilleure technologie disponible et économiquement acceptable. Certains pays proposent ou ont déjà décidé des réductions de 4 à 5 dBA sur les automobiles, de 3 à 8 dBA sur les motocyclettes et de 5 à 10 dBA sur les véhicules lourds (camions et véhicules de transport en commun) pour la période 19851990. Toutefois, des réductions supplémentaires seront nécessaires à l'avenir afin de procurer un environnement acoustique satisfaisant. Par conséquent, les pays Membres devraient encourager et, le cas échéant, aider financièrement la recherche et le développement de nouvelles technologies de lutte contre le bruit, afin de faciliter un nouvel abaissement des niveaux d'émission dans le futur. II sera également nécessaire d'améliorer les procédures de mesure du bruit des véhicules à moteur afin d'établir un lien plus étroit entre les limites fixées et le bruit effectivement émis.

Compte tenu de cette situation et considerant la lenteur de renouvellement du parc automobile ainsi que de la nécessité d'informer suffisamment à l'avance les constructeurs automobiles, il faudrait que les instances appropriées décident, dès que possible, et avec le plein appui des pays Membres de l'OCDE, une réduction des niveaux d'émission sonore des véhicules à moteur de 5 à $10 d B A$ selon le type de véhicule(5). Cette réduction devrait être mise en œuvre entre 1985 et 1990 afin que les effets s'en fassent pleinement sentir en l'an 2000.

En outre, la gestion de la circulation constitue un moyen important pour lutter contre le bruit, réduire la pollution de l'air et pour économiser l'énergie.

Au cours de la conférence, la délégation américaine a proposé d'organiser, aux États-Unis avant la fin de l'année, une réunion spéciale destinée à explorer les dispositions propres à assurer une harmonisation internationale de procédures de mesure du bruit, en particulier celui des véhicules à moteur. Cette proposition a retenu toute l'attention des pays Membres.

(1) Voir l'Observateur de l'OCDE $N^{\circ} 103$, mars 1980.

12) Les autres conclusions portent notamment sur des mesures à caractère préventif et incitatif, le bruit des avions, I'information et l'éducation, la compensation, la coopération et l'harmonisation internationales.

(3) L'échelle Ldn est un indice de bruit utilisé aux États-Unis : il correspond à la moyenne jour/nuit du niveau $L e q$, avec pondération aggravante de 10 dBA pour la période "nuit \# (de 22 heures à 7 heures).

(4) Au Japon, les limites sont des maxima sans tolérances de dépassement alors que dans les Communautés européennes une tolérance de $1 \mathrm{dBA}$ est admise.

(5) A titre d'exemple de réduction, on peut citer les limites proposées par le gouvernement de la RFA aux Communautés européennes pour 1985: 75 dBA pour les voitures, et 80 dBA pour les poids lourds et véhicules de transports en commun. 


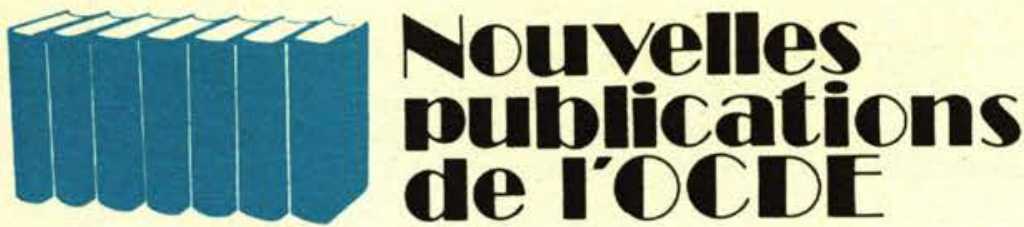

Prix de vente au public à la librairie du siège de l'OCDE

"Études économiques de I'OCDE „ Série 1980 :

Études annuelles, très détaillées, pour chaque pays de l'OCDE, des tendances et de la politique économiques.

ESPAGNE (avril 1980)

(1080 242) ISBN $926422047 \times 74$ pages TUROUIE (mai 1980)

(1080 272) ISBN 926422066666 pages YOUGOSLAVIE (mai 1980)

(108041 2) ISBN 926422075590 pages

PAYS-BAS (mars 1980)

(1080 202) ISBN 926422031396 pages

SUĖDE (avril 1980)

(1080252) ISBN 926422061598 pages Lo numéro …… F14,00 \& 1.60 US $\$ 3.50$ ISSN 03043363 ... F/4,00 L1.60 US\$3.50

Abonnement ….F F240.00 ₹26.60 US $\$ 60.00$

$A$ paraitre en juillet/aoQt :

L'ACIER DANS LES ANNÉES 80.

Symposium, Paris, février 1980 (juillet/aoat 1980)

(Voir l'Observateur de l'OCDE, $n^{\circ} 103$, mars 1970).

(58 8002 2) ISBN $926422081 X$

$F 90,00 £ 10.00$ US $\$ 22.50$

L'INDUSTRIE SIDÉRURGIQUE

EN 1978 (avril 1980)

(58 8001 3) ISBN 9264020586

40 pages, bilingue .... F18,00 ₹ 2.00 US $\$ 4.50$ (mai 1980) Série "Documents" (9780042) ISBN 926422063 1

180 pages ......... F $40,00 £ 4.40$ US $\$ 10.00$

"Études du Centre de développement : :

LA PARTICIPATION DE LA POPULATION AUX SYSTEMMES D'APPROVISIONNEMENT D'EAU

EN MILIEU RURAL (avril 1980)

Série "Documents"

(4180012) ISBN 9264220275

176 pages ........... F36,00 ₹4.00 US\$9.00

OFFRES A PRIX EXCEPTION-

NELS ET AUTRES PRATIQUES COMMERCIALES ANALOGUES

(mai 1980) Série "Documents"

(2480012) ISBN $926422033 X$

66 pages ...........F F24.00 £2.70 us $\$ 6.00$

EXAMEN DES POLITIQUES

NATIONALES D'ÉDUCATION :

DANEMARK (juin 1980)

$(9180032$ 2) ISBN 926422071

184 pages........ F42,00 £ 4.70 us $\$ 10.50$

SÉCURITÉ ROUTIÈRE DE NUIT (mai 1980)

(778003 2) ISBN 9264220690

110 pages .........F F $40,00 € 4.40$ US $\$ 10.00$

PROBLÈMES DE CAPACITE

INFRASTRUCTURELLE POSÉS

PAR LE TRANSIT INTERNA-

TIONAL. 45ème Table ronde CEMT (avril 1980)

(758002 2) ISBN 9282120597

156 pages ........F F18,00 £ 2.00 US $\$ 4.50$

COMPTES NATIONAUX DES

PAYS DE L'OCDE, 1950-1978.

Vol. I. Principaux agrégats

(avril 1980)

(308001 3) ISBN 9264020594

90 pages, bilingue .... F F30,00 £ 3.30 US $\$ 7.50$
STATISTIQUES DE L'ÉNERGIE, 1974-1978 (avril 1980)

(618002 3) ISBN 9264020578

316 pages, bilingue F60,00 $\mathrm{E} 6.70$ US $\$ 15.00$

BILANS ENERGETIQUES

DES PAYS DE L'OCDE, 1974-

1978 (mars 1980)

(618001 3) ISBN $926402056 \times$

170 pages, bilingue . F $36,00 £ 4.00$ US $\$ 9.00$

STATISTIQUES - TRIMES-

TRIELLES DU PÉTROLE. QUatrième trimestre 1979. $\mathrm{N}^{\circ} \mathrm{I} / 1980$ (mai 1980)

(6080013) 292 pages, billingue Le numéro ........F60,00 ₹6.70 US\$15.00 ISSN 0378 6536. Abonnement 1980

F200,00 $₹ 22.00$ US $\$ 50.00$

L'INDUSTRIE CHIMIQUE, 1978 (juin 1.980)

(598001 3) ISBN 9264020918

56 pages, bilingue ... F $26,00 £ 2.90$ us $\$ 6.50$

BILANS DE LA VIANDE DANS

LES PAYS DE L'OCDE, $1973 / 1978$ - avril 1980 (mai 1980) Série "Documents"

(51 8005 3) ISBN $926402090 X$

86 pages, bilingue ... F $F 30,00$ £ 3.30 US $\$ 7.50$

PATES ET PAPIERS. Statistiques trimestrielles. Quatrième trimestre 1979. $\mathrm{N}^{\circ} \mathrm{I} / 1980$ (mai 1980)

56 pages, bilingue

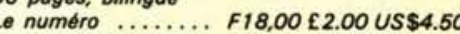
ISSN $0335377 X$. Abonnement

F54.00 ₹ 6.00 US $\$ 13.50$

TENDANCES DES MARCHES DES CAPITAUX N० 13 février 1980 (juin 1980)

$12780012) 126$ pages

Le numéro ........F. F28,00 £3.10 us\$7.00 ISSN 03786529 Abonnement 15 numeros par an) .......F F120,00 £ 13.30 US $\$ 30.00$

\section{LE NOUVEAU CENTRE DE BONN}

Un nouveau centre de publications et d'information de I'OCDE a été créé à Bonn pour desservir la région centrale d'Europe, en particulier les pays Membres germanophones. II a été officiellement inauguré le $\mathbf{2 5}$ juin par le Secrétaire général de I'OCDE, Emile van Lennep.

Lors de la cérémonie d'ouverture, à laquelle assistaient Otto Graf Lambsdorff, ministre des Affaires économiques de la RFA, d'autres personnalités allemandes, la presse et des ambassadeurs de pays Membres de l'OCDE, le Secrétaire général a tenu les propos suivants : "L'ouverture du centre de Bonn marque une nouvelle étape dans les efforts que nous

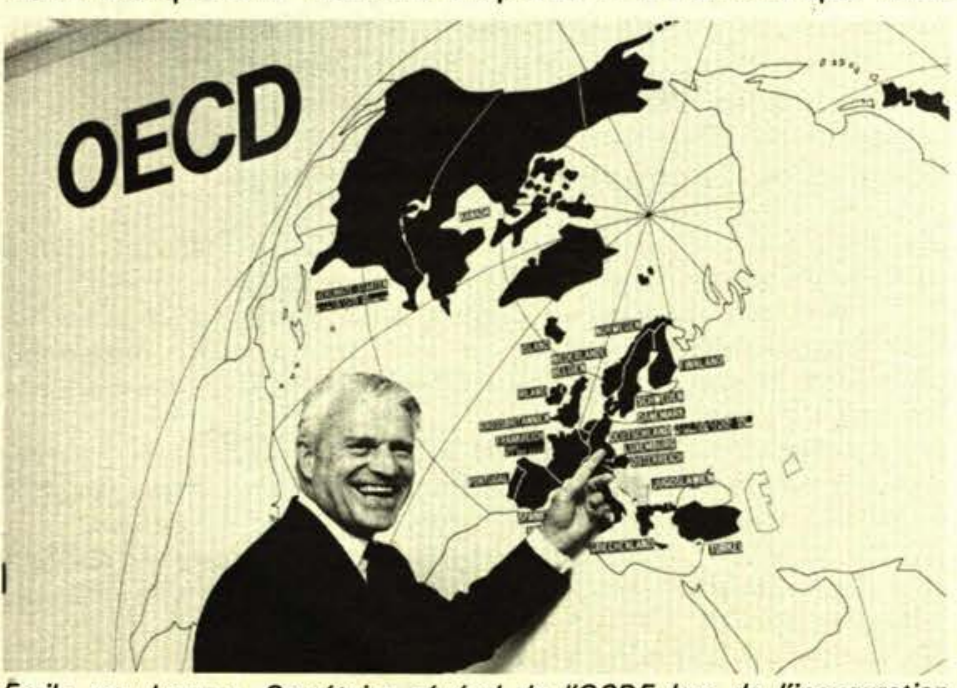

Emile van Lennep, Secrétaire général de l'OCDE, lors de l'inauguration du Centre de Bonn déployons pour aider les pays Membres à faire mieux comprendre à l'opinion publique les politiques et les priorités qu'ils définissent dans le cadre de l'OCDE. Les gouvernements des pays Membres nous incitent de plus en plus à accélérer la diffusion des travaux de l'Organisation - ses analyses, ses recommandations. Nous avons entrepris de le faire en intensifiant les programmes d'information et de publications avec le concours, notamment, des centres existants de Washington et de Tokyo. Maintenant, le Conseil a décidé d'élargir le champ de nos efforts en permettant l'établissement du centre régional de Bonn créé à l'intention de tous les segments de la population - parlementaires, journalistes, universitaires, organisations d'employeurs et de travailleurs."

Otto Graf Lambsdorff a, quant à lui, souligné le rôle de plus en plus important que joue I'OCDE. "L'Organisation contribue de manière appréciable à répondre aux besoins sans cesse croissants d'information, de consultation et d'action qui se font jour dans les pays industrialisés du monde occidental. A cet égard, le nouveau centre est appelé, par ses activités dans le domaine de l'information et des publications, à assumer une tâche essentielle.»

Le centre est situé au 4 Simrockstrasse, 5300 Bonn; numéro de téléphone : $216045-8$. II est dirigé par Dieter Menke.

Toutes les publications de l'Organisation peuvent être commandées au centre qui, en outre, met une salle de lecture à la disposition de ceux qui désirent les consulter. Les journalistes allemands et les correspondants étrangers stationnés à Bonn peuvent y écouter en direct des transmissions de conférences de presse données au siège de l'OCDE à Paris. 


\section{Où obtenir les publications de l'OCDE}

ALLEMAGNE

OECD Publications and Information Center, 4 Simrockstrasse,

5300 Bonn

Tél. (0228) 216045

ARGENTINE

Carlos Hirsch S.R.L.,

Florida $165,4^{\circ}$ Piso,

(Galeria Guemes) 1333 Buenos Aires

Tél. 33-1787-2391 Y 30-7122

\section{AUSTRALIE}

Australia and New Zealand Book Co. Pty. Ltd., 23 Cross St. (P.O.B. 459)

Brookvale, New South Wales, 2100

Tél. 938.22.44

\section{AUTRICHE}

OECD Publications and Information Center, 4 Simrockstrasse,

5300 Bonn (Allemagne). Tél. (0228) 216045

Agent local:

Gerold \& Co., Graben 31, Wien 1.

Tẻl. 52.22 .35

BELGIQUE

LCLS,

44 rue Otlet, 1070 Bruxelles.

Tél. 02-521 2813

\section{BRÉSIL}

Mestre Jou S.A.,

Rua Guaipá 518, Caixa Postal 24090,

05089 Sao Paulo 10

Tél. 261.1920

Rua Senador Dantas $19 \mathrm{~s} / 205-6$,

Rio de Janeiro - G.B.

Tél. 232.07.32

CANADA

Renouf Publishing Company Limited,

2182 St. Catherine Street West,

Montréal, Québec H3H 1 M7.

Tél. (514) 937.3519

CORÉE

Pan Korea Book Corporation

P.O. Box $\mathrm{n}^{\circ} 101$, Kwangwhamun, Séoul.

Tél. 72.7369

\section{DANEMARK}

Munksgaards Boghandel,

Nørregade 6, DK-1165 København K. Tél. (01) 12.85 .70

ESPAGNE

Mundi-Prensa Libros, S.A.

Castello 37, Apartado 1223, Madrid 1. Tél. 275.46.55/276.02.53

Libreria Bastinos de José Bosch,

Pelayo 52, Barcelona 1. Tél. 222.06.00

ÉTATS-UNIS

OECD Publications and Information Center,

Suite 1207, 1750 Pennsylvania Ave. N.W.,

W ashington, D.C. 20006.

Tél. (202) 724.1857

FINLANDE

Akateeminen Kirjakauppa,

Keskuskatu 1, 00100 Helsinki 10.

Tél. 65.11 .22

FRANCE

Bureau des Publications de l'OCDE

2 rue André-Pascal, F 75775 Paris Cedex 16.

Principal correspondant:

Tél. (1) 524.81 .67

Librairie de l'Université,

13602 Aix-en-Provence.

Tél. (42)26.18.08

GRĖCE

Librairie Kauffmann,

rue du Stade, Athènes 132.

Tél. 322.21 .60
HONG KONG

Government Information Services,

Sales and Publications Office,

Beaconsfield House, 1st floor,

Queen's Road Central.

Tél. 5-233191

INDE

Oxford Book and Stationery Co.,

Scindia House, New Delhi I.

Tél. 45896

17 Park St., Calcutta.

Tél. 240832

ISLANDE

Snaebjörn Jónsson \& Co., h.f.,

Hafnarstraeti 4 \& 9 ,

P.O.B. 1131 - Reykjavik.

Tél. 13133/14281/11936

ITALIE

Libreria Commissionaria Sansoni,

Via Lamarmora 45, 50121 Firenze.

Tél. $579751 / 2 / 3$

Via Bartolini 29, 20155 Milano. Tél. 365083

Sub-depositari:

Editrice e Libreria Herder,

Piazza Montecitorio 120, 00186 Roma

Tél. 6794628

Libreria Hoepli,

Via Hoepli 5, 20121 Milano. Tél. 865446

Libreria Lattes,

Via Garibaldi 3, 10122 Torino. Tél. 519274

La diffusione delle edizioni OCSE è inoltre assicurata dalle migliori librerie nelle città più importanti.

\section{JAPON}

OECD Publications and Information Center,

Landic Akasaka Bldg., 2-3-4 Akasaka,

Minato-ku, Tokyo 107.

Tél. 586-2016

LIBAN

Documenta Scientifica/Redico,

Edison Building, Bliss St.,

P.O.B. 5641, Beirut. Tél. 354429-344425

MALAISIE

University of Malaya Co-operative Bookshop

Ltd.,

P.O.Box 1127, Jalan Pantai Baru,

Kuala Lumpur. Tél. 51425, 54058, 54361

NORVÈGE

J.G. Tanum A/S,

$\mathrm{K}$ arl Johansgate $41 / 43$, Oslo 1.

$$
\text { Tél. (02) } 801260
$$

NOUVELLE-ZÉLANDE

The Publications Manager,

Government Printing Office,

Wellington: Mulgrave Street (Private Bag),

World Trade Centre, Cubacade, Cuba Street, Rutherford House, Lambton Quay.

Tél. 737.320

Auckland: Rutland Street (P.O. Box 5344) Tél. 32.919

Christchurch: 130 Oxford Tce, (Private Bag). Tél. 50.331

Hamilton: Barton Street (P.O. Box 857). Tél. 80.103

Dunedin: T. \& G. Building, Princes Street,

(P.O. Box 1104).

Tél. 78.294

PAKISTAN

Mirza Book Agency,

65 Shahrah Quaid-E-Azam, Lahore 3.

Tél. 66839
PAYS-BAS

Staatsuitgeverij

Verzendboekhandel

Chr. Plantijnstraat

'S-Gravenhage:

Tél. 070-789911

Voor bestelligeen:

Tél. 070-789208

PORTUGAL

Livraria Portugal,

Rua do Carmo 70-74, 1117 Lisboa Codex.

Tél. $360582 / 3$

ROYAUME-UNI et

COLONIES DE LA COURONNE

H.M. Stationery Office,

P.O.B. 569, London SE1 9NH

Tél. 01.928.6977, Ext. 410

or

49 High Holborn

London WC 1V 6HB (personal callers)

Branches at: Belfast Birmingham, Bristol, Cardiff, Edinburgh, Manchester.

SUÈDE

AB CE Fritzes Kungl. Hovbokhandel,

Box 16 356, S 10327 STH, Regeringsgatan 12,

DS Stockholm. Tél. 08/23.89.00

SUISSE

OECD Publications and Information Center, 4 Simrockstrasse,

5300 Bonn (Allemagne). Tél. (0228) 216045

Agent local:

Librairie Payot,

6 rue Grenus, 1211 Genève 11 .

Tél. 022-31.89.50

TAIWAN-FORMOSE

National Book Company,

84-5 Sing Sung Rd., Sec. 3,

Taipei 107.

Tél. 321-0698

THAILANDE

Suksit Siam Co., Ltd.,

1715 Rama IV Rd.,

Samyam Bangkok 5.

Tél. 2511630

VENEZUELA

Libreria del Este,

Avda F. Miranda 52, Aptdo. 60337,

Edificio Galipan, Caracas 106. Tél. 32.23.01/33.26.04/33.24.73

YOUGOSLAVIE

Jugoslovenska Knjiga, Terazije 27,

P.O.B. 36, Beograd.

Tél. 621.992

Les commandes en provenance de pays où I'OCDE n'a pas encore désigné de dépositaire peuvent être adressées au Bureau des Publications de l'OCDE, 2 rue André-Pascal, F75775 PARIS CEDEX 16. 
Organisation de Coopération et de Développement Economiques

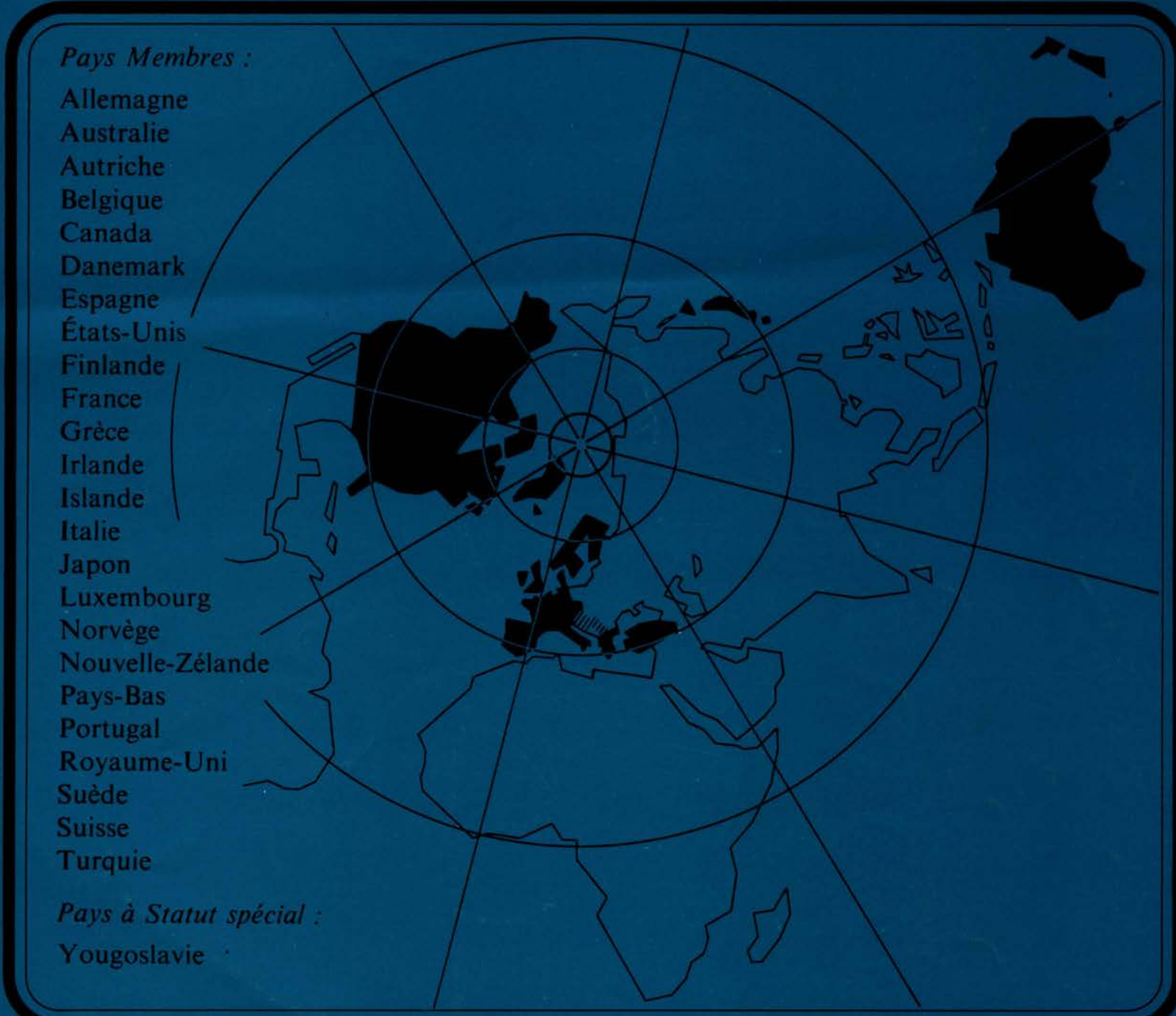

